\title{
DISCRETE DUALITY FINITE VOLUME SCHEMES FOR DOUBLY NONLINEAR DEGENERATE HYPERBOLIC-PARABOLIC EQUATIONS
}

\author{
B. ANDREIANOV, M. BENDAHMANE, AND K. H. KARLSEN
}

\begin{abstract}
We consider a class of doubly nonlinear degenerate hyperbolicparabolic equations with homogeneous Dirichlet boundary conditions, for which we first establish the existence and uniqueness of entropy solutions. We then turn to the construction and analysis of discrete duality finite volume schemes (in the spirit of Domelevo and Omnès [41) for these problems in two and three spatial dimensions. We derive a series of discrete duality formulas and entropy dissipation inequalities for the schemes. We establish the existence of solutions to the discrete problems, and prove that sequences of approximate solutions generated by the discrete duality finite volume schemes converge strongly to the entropy solution of the continuous problem. The proof revolves around some basic a priori estimates, the discrete duality features, Minty-Browder type arguments, and "hyperbolic" $L^{\infty}$ weak- $\star$ compactness arguments (i.e., propagation of compactness along the lines of Tartar, DiPerna, ...). Our results cover the case of non-Lipschitz nonlinearities.
\end{abstract}

\section{CONTENTs}

1. Introduction

2. Notions of solution and well-posedness 5

3. Discrete duality finite volume (DDFV) schemes 13

3.1. Construction of "double" conformal meshes 14

3.2. Mesh parameters and regularity of meshes 16

3.3. Discrete gradient and divergence operators 17

3.4. Penalization operator 19

3.5. Discrete convection operator 20

3.6. Projection operators and test functions 21

3.7. Dependency on $t$ and further notation 21

3.8. The finite volume scheme 22

4. Elements of discrete calculus for DDFV schemes 23

Date: January 7, 2009.

2000 Mathematics Subject Classification. Primary 35K65, 74S10; Secondary 35A05, 65M12.

Key words and phrases. Degenerate hyperbolic-parabolic equation, conservation law, LerayLions type operator, non-Lipschitz flux, entropy solution, existence, uniqueness, finite volume scheme, discrete duality, convergence.

The work of M. Bendahmane was supported by the FONDECYT project 1070682. The work of K. H. Karlsen was supported by the Research Council of Norway through an Outstanding Young Investigators Award. A part of this work was done while B. Andreianov enjoyed the hospitality of the Centre of Mathematics for Applications (CMA) at the University of Oslo, Norway. This article was written as part of the the international research program on Nonlinear Partial Differential Equations at the Centre for Advanced Study at the Norwegian Academy of Science and Letters in Oslo during the academic year 2008-09. 
4.1. Discrete duality formulas for the diffusion terms 23

4.2. Summation formulas for the penalization terms 25

4.3. Discrete duality formulas for the evolution terms 25

4.4. Discrete dualitv formulas for the convection terms 25

5. Properties of discrete operators and functional spaces 27

5.1. Discrete functions and fields as elements of Lebesgue spaces 28

5.2. Consistency properties of discrete operators 28

5.3. Discrete embedding and compactness results $\quad 29$

6. Properties of discrete solutions 31

6.1. A priori estimates 31

6.2. Existence of discrete solutions 36

6.3. Discrete entropy inequalities 37

6.4. Control of the remainder terms in Proposition $6.3 \quad 38$

6.5. Approximate continuous entropv inequalities 40

7. Convergence and statement of main result 41

8. On the choice of FV scheme and various generalizations 44

Appendix A: Proof of uniqueness 47

Appendix B: The reconstruction property $\quad 55$

$\begin{array}{ll}\text { References } & 56\end{array}$

\section{INTRODUCTION}

In this paper we consider degenerate hyperbolic-parabolic problems of the form

$$
\begin{cases}\partial_{t} u+\operatorname{div} \mathfrak{f}(u)-\operatorname{div} \mathfrak{a}(\nabla A(u))=\mathcal{S}, & \text { in } Q:=(0, T) \times \Omega, \\ \left.u\right|_{t=0}=u_{0}, & \text { in } \Omega \\ u=0, & \text { on } \Sigma=(0, T) \times \partial \Omega,\end{cases}
$$

where $u:(t, x) \in Q \rightarrow \mathbf{R}$ is the unknown function, $T>0$ is a fixed time, $\Omega \subset \mathbf{R}^{d}$ is a bounded domain with polygonal boundary $\partial \Omega$ and outward unit normal $n$. We consider the cases $d=2$ and $d=3$. The initial data $u_{0}: \Omega \rightarrow \mathbf{R}$ are assumed to be a bounded measurable function, i.e.,

$$
u_{0} \in L^{\infty}(\Omega)
$$

while the source $\mathcal{S}: Q \rightarrow \mathbf{R}$ is assumed to be a measurable function for which $\mathcal{S}(t, \cdot) \in L^{\infty}(\Omega)$ for a.e. $t \in(0, T)$ and $\int_{0}^{T}\|\mathcal{S}(t, \cdot)\|_{L^{\infty}(\Omega)} d t<\infty$; we abusively denote it by

$$
\mathcal{S} \in L^{1}\left(0, T ; L^{\infty}(\Omega)\right)
$$

The function $\mathfrak{a}: \mathbf{R}^{N} \rightarrow \mathbf{R}^{N}$ is taken under the form

$$
\mathfrak{a}(\xi)=k(\xi) \xi,
$$

where $k$ is a scalar function. The function $\mathfrak{a}$ is assumed to be continuous and strictly monotone. We assume that there exist $p \in(1,+\infty)$ and $C>0$ such that

$$
\frac{1}{C}|\xi|^{p-2} \leq k(\xi) \leq C|\xi|^{p-2}, \quad \forall \xi \in \mathbf{R}^{d} \backslash\{0\} .
$$


In particular, the associated operator $w \mapsto-\operatorname{div}(k(|\nabla w|) \nabla w)$ is a Leray-Lions operator acting from $W_{0}^{1, p}(\Omega)$ to $W^{-1, p^{\prime}}(\Omega)$ with $p^{\prime}=\frac{p}{p-1}$. A prototype example is the $p$-laplacian, which corresponds to $k(\xi)=|\xi|^{p-2}$.

We assume that the diffusion function $A(\cdot)$ satisfies

$$
A(\cdot) \text { is continuous and nondecreasing, normalized by } A(0)=0 \text {, }
$$

while the convective flux function $\mathfrak{f}(\cdot)$ satisfies

$$
\mathfrak{f}=\left(\mathfrak{f}_{1}, \ldots, \mathfrak{f}_{d}\right): Q \times \mathbf{R} \rightarrow \mathbf{R}^{d} \text { is continuous and normalized by } \mathfrak{f}(0)=0 .
$$

We emphasize that the fluxes $\mathfrak{f}, A$ are not necessarily locally Lipschitz continuous.

Problems more general than (10), for which our results can be extended, will be discussed in Section 8 .

The class (10) of nonlinear partial differential equations includes several important particular cases. The hyperbolic conservation law

$$
\partial_{t} u+\operatorname{div} \mathfrak{f}(u)=0
$$

is a special case of (10). The celebrated theory of $L^{\infty}$ entropy solutions for scalar conservation laws in $\mathbf{R}^{d}$ was developed by Kruzhkov [63, while the $B V$ theory was set up by Vol'pert 777. The extensions for the Dirichlet problem in bounded domains are due to Bardos, LeRoux, Nédélec [15] (for the $B V$ setting) and Otto 69] (for the $L^{\infty}$ setting). Note that the boundary condition is only verified in some generalized sense (see [15, 69, 65, 29, 73, 66, 79, 49, 67, 55).

Many other well-known partial differential equations (usually possessing more regular solutions) are also special cases of (11). Let us mention the heat and porous medium equations

$$
\partial_{t} u=\Delta u, \quad \partial_{t} u=\Delta u^{m}, \quad m>1,
$$

and more generally degenerate convection-diffusion equations of the type

$$
\partial_{t} u+\operatorname{div} \mathfrak{f}(u)=\Delta A(u) .
$$

Degenerate parabolic equations like (3) occur in theories of flow in porous media (see discussion and references [43) and sedimentation-consolidation processes [27.

As other famous representatives of the class of equations that is considered herein, we mention the $p$-Laplace equation

$$
\partial_{t} u=\operatorname{div}\left(|\nabla u|^{p-2} \nabla u\right), \quad p>1,
$$

which arises in the theory of non-Newtonian filtration. Also well known is the more general polytropic filtration equation

$$
\partial_{t} u=\operatorname{div}\left(\left|\nabla\left(|u|^{m-1} u\right)\right|^{p-2} \nabla\left(|u|^{m-1} u\right)\right), \quad m, p>1 .
$$

A related class of equations consists of the so-called elliptic-parabolic equations

$$
\partial_{t} b(v)=\operatorname{div} \mathfrak{a}(v, \nabla v),
$$

where $b: \mathbf{R} \rightarrow \mathbf{R}$ is continuous nondecreasing, and $\mathfrak{a}(r, \xi): \mathbf{R} \times \mathbf{R}^{N} \rightarrow \mathbf{R}^{N}$ gives rise to a Leray-Lions operator. We refer to [4, 20, 70, 30, 6] and the references cited therein for more information on elliptic-parabolic equations.

A chief goal of this paper is to propose and analyze a specific class of finite volume schemes for the problem (10). Note that finite volume schemes are well suited for approximation of equations in divergence form, such as (1). Discretization of 
the aforementioned hyperbolic, porous medium, convection-diffusion, and ellipticparabolic equations by finite volume methods is quite standard by now and often used in engeneering practice. We refer to [48, 31, 3, 44, 45, 61, 57, 68, 79, 49, 67, 12, 10, 11, 42 and references therein for different convergence results and numerical experiments. For related works on linear elliptic problems, see 2, 1, 57, 41, 23, 58, 50, 51, 53, 52 and the discussion in Section 8. Alternative numerical approaches have also been investigated; here we only mention finite element schemes (see 36, [16] and references therein), kinetic schemes (see [14, 22, 55] and references therein) and operator splitting schemes (see 43]).

Having said that, we are not aware of any papers that construct convergent numerical schemes for mixed type equations of the generality considered herein. Indeed, they combine a number of difficulties such as nonlinear convection, doubly nonlinear diffusion, strong degeneracy, and shocks, which in turn necessitates the use of a suitable framework of discontinuous entropy solutions. Furthermore, in the absence of the Lipschitz continuity assumption on the convective flux $\mathfrak{f}(\cdot)$, the CFL condition does not make sense; therefore we have to discretize the convective term with a time-implicit scheme.

We begin by providing the entropy solution framework for (1); this is the topic of Section 2 and Appendix A. Due to the nonlinearity of $\mathfrak{f}(\cdot)$ and the possible degeneracy of $A(\cdot)$, the problem (11) will in general possess shock wave solutions, a feature that can reflect the physical phenomenon of breaking of waves. This is well known in the context of conservation laws. Also the boundary condition cannot be prescribed pointwise on the whole boundary $\Sigma$ when $A$ is not strictly increasing. Due to this loss of regularity, it is necessary to work with weak solutions; moreover, to single out a physically relevant and unique weak solution, we need to impose additional "entropy inequalities", in the spirit of Kruzhkov [63]. Early results on hyperbolic-parabolic equations were obtained by Volpert, Hudjaev [78; see also [80, 82, 81, 74, 28, 19] and references cited therein, and 76, 37, 32. $L^{1}$ entropy techniques for degenerate convection-diffusion equations like (3), which take into account both hyperbolic and parabolic features, were developed by Carrillo 29] for the homogeneous Dirichlet problem in bounded domains. Since then, many authors extended the Carrillo results in various directions (see e.g. 30, 59, 66, 73, 25, 46, 60, 62, 67, 49, 5, 13]). Some additional techniques are required for anisotropic diffusion problems, where a kinetic approach (see Chen, Perthame [35]) and an accurate entropic approach (see Bendahmane, Karlsen [17. [18) were developed in the few last years; see also Souganidis, Perthame [75] and and Chen, Karlsen [34. In this paper, we use a variant of the Carrillo entropic approach. Following the Tartar-DiPerna idea of measure-valued solutions and using the techniques of Eymard, Gallouët, Herbin [48, we introduce a notion of entropy process solution for (1), and establish the related identification and uniqueness results. More exactly, we work with entropy double-process solutions arising in the particular context of discrete duality finite volume schemes. In Section 2, we show the existence result for (10) and state uniqueness; an adaptation of the standard uniqueness (and, more generally, $L^{1}$ contraction and comparison principle) proof is given in Appendix A.

In Section 3 we construct discrete duality finite volume (DDFV) schemes for (11) in two and three spatial dimensions (some other schemes are briefly discussed in Section (8). We adapt the approximations used by Eymard, Gallouët, Herbin 
48. (see also [31, 79, 49, 67]) for the nonlinear convection term, and those used by Hermeline [57, 58, Domelevo, Omnès [41] and Andreianov, Boyer, Hubert [1] for the doubly nonlinear diffusion term. In $3 \mathrm{D}$, we propose new DDFV schemes that possess convenient discrete duality properties.

Our 3D scheme is a very particular case of the schemes introduced and studied numerically by Hermeline in 58. In passing, we mention that different kinds of $3 \mathrm{D}$ discrete duality schemes were constructed in 72,39 and in 38. Appendix B (see also [8, 7]) is devoted to an elementary reconstruction lemma which underlies our DDFV schemes in 3D. In contrast to [1, 11, we are led to penalize our DDFV schemes to ensure that the two approximations of $A(u)$ actually converge to the same limit (see Section 3.4). The DDFV schemes constructed in Section 3 possess several convenient discrete calculus formulas that we collect in Section 4 Related consistency estimates and properties of the associated spaces of discrete functions are given in Section 5. The (few) available a priori estimates for the discrete solutions are collected in In Section [6. In the same section, the existence of discrete solutions is shown. Furthermore, we establish that, up to an error term in the equation depending on the discretization parameter, discrete solutions can be considered as entropy solutions of (1). In Section 7 we prove that discrete solutions converge, as the discretization parameter tends to zero, to an entropy double-process solution that turns out to be the (unique) entropy solution of (11). It should emphasized that we obtain strong convergence of both convective and diffusive fluxes, in spite of the double nonlinearity of the problem (11). Section 8 contains references to some known finite volume schemes for nonlinear diffusion-convection equations, and discusses the extension of our results to different generalizations of problem (11).

\section{Notions of SOLUTION AND WELL-POSEDNESS}

As it was explained in the introduction, we need the notion of weak solution for (1) with additional "entropy" conditions. In order to use entropy conditions in the interior of $Q$ and, moreover, take into account the homogeneous Dirichlet boundary condition on $\Sigma$, following Carrillo 29 we will work with the so-called "semi-Kruzhkov" entropy-entropy flux pairs $\left(\eta_{c}^{ \pm}, \mathfrak{q}_{c}^{ \pm}\right)$for each $c \in \mathbf{R}$; they are defined as

$$
\begin{aligned}
\eta_{c}^{+}(z)=(z-c)^{+}, & \eta_{c}^{-}(z)=(z-c)^{-} \\
\mathfrak{q}_{c}^{+}(z)=\operatorname{sign}^{+}(z-c)(\mathfrak{f}(z)-\mathfrak{f}(c)), & \mathfrak{q}_{c}^{-}(z)=\operatorname{sign}^{-}(z-c)(\mathfrak{f}(z)-\mathfrak{f}(c)) .
\end{aligned}
$$

By convention, we assign $\left(\eta_{c}^{ \pm}\right)^{\prime}(c)$ to be zero. Here $(z-c)^{ \pm}$denote the nonnegative quantities satisfying $z-c=(z-c)^{+}-(z-c)^{-}$; moreover, we use the notation

$$
\begin{aligned}
& \operatorname{sign}^{+}(z-c)=\left(\eta_{c}^{+}\right)^{\prime}(z)= \begin{cases}1, & z>c \\
0, & z \leq c,\end{cases} \\
& \operatorname{sign}^{-}(z-c)=\left(\eta_{c}^{-}\right)^{\prime}(z)= \begin{cases}0, & z \geq c, \\
-1, & z<c .\end{cases}
\end{aligned}
$$

At certain points, we will also need smooth regularizations of the semi-Kruzhkov entropy-entropy flux pairs; it is sufficient to consider regular "boundary" entropy pairs $\left(\eta_{c, \varepsilon}^{ \pm}, \mathfrak{q}_{c, \varepsilon}^{ \pm}\right)$(cf. Otto 69 and the book 65]), which are $W^{2, \infty}$ pairs with the same support as $\left(\eta_{c}^{ \pm}, \mathfrak{q}_{c}^{ \pm}\right)$, converging pointwise to $\left(\eta_{c}^{ \pm}, \mathfrak{q}_{c}^{ \pm}\right)$as $\varepsilon \rightarrow 0$. Specifically, 
the functions

$$
\operatorname{sign}_{\varepsilon}^{+}(z)=\frac{1}{\varepsilon} \min \left\{z^{+}, \varepsilon\right\}, \quad \operatorname{sign}_{\varepsilon}^{-}(z)=\frac{1}{\varepsilon} \max \left\{-z^{-},-\varepsilon\right\}
$$

will be used to approximate $\operatorname{sign}^{ \pm}(\cdot)=\left(\eta_{0}^{ \pm}\right)^{\prime}(\cdot)$.

In view of the monotonicity of $A: \mathbf{R} \rightarrow \mathbf{R}$, the following definition is meaningful.

Definition 2.1. For any locally bounded piecewise continuous function $\theta: \mathbf{R} \rightarrow \mathbf{R}$, we define (using, e.g., the Stieltjes integral) the function $A_{\theta}: \mathbf{R} \rightarrow \mathbf{R}$ by

$$
A_{\theta}(z)=\int_{0}^{z} \theta(s) d A(s) .
$$

The ensuing lemma shows that there exists a continuous function $\widetilde{A}_{\theta}$ such that $A_{\theta}(z)=\widetilde{A}_{\theta}(A(z))$. We prove this lemma under rather strong assumptions, but they are still sufficient for our needs.

Lemma 2.1. (i) Let $\theta, A_{\theta}$ be a couple of functions as introduced in Definition 2.1. Then there exists a continuous function $\widetilde{A}_{\theta}: A(\mathbf{R}) \rightarrow \mathbf{R}$ such that

$$
A_{\theta}(z)=\widetilde{A}_{\theta}(A(z)), \quad \forall z \in \mathbf{R} .
$$

Moreover, $\widetilde{A}_{\theta}$ is Lipschitz continuous.

(ii) Assume additionally that $\theta \in W^{1, \infty}(\mathbf{R})$, and let $\left(A^{\rho}\right)_{\rho}$ be a sequence of nondecreasing continuous surjective functions converging to $A$ pointwise on $\mathbf{R}$ as $\rho \rightarrow 0$. Define $\widetilde{A}_{\theta}^{\rho}, A_{\theta}^{\rho}$ by (i) and (4) with $A^{\rho}$ replacing $A$. Then $\widetilde{A}_{\theta}^{\rho}$ converges to $\widetilde{A}_{\theta}$ uniformly on compact subsets of $A(\mathbf{R})$.

Proof. (i) For $b \in A(\mathbf{R})$, we can define $\widetilde{A}_{\theta}$ by $\widetilde{A}_{\theta}(b)=A_{\theta}(z)$ for some $z \in A^{-1}(b)$. If $A(z)=A(\hat{z})$, then the measure $d A(s)$ vanishes between $z$ and $\hat{z}$; thus

$$
A_{\theta}(z)-A_{\theta}(\hat{z})=\int_{\hat{z}}^{z} \theta(s) d A(s)=0,
$$

and $\widetilde{A}_{\theta}$ is well-defined. For all $b, \hat{b} \in A(\mathbf{R})$,

$$
\widetilde{A}_{\theta}(b)-\widetilde{A}_{\theta}(\hat{b})=A_{\theta}(z)-A_{\theta}(\hat{z})=\int_{\hat{z}}^{z} \theta(s) d A(s), \quad z \in A^{-1}(b), \hat{z} \in A^{-1}(\hat{b}) .
$$

Consequently,

$$
\left|\widetilde{A}_{\theta}(b)-\widetilde{A}_{\theta}(\hat{b})\right| \leq\|\theta\|_{L^{\infty}}|A(z)-A(\hat{z})|=\|\theta\|_{L^{\infty}}|b-\hat{b}| .
$$

(ii) Since the functions $\widetilde{A}_{\theta}^{\rho}$ are monotone, by the Dini theorem it is sufficient to prove the pointwise convergence. By the same argument, the convergence of $A^{\rho}$ to $A$ is actually uniform on compact subsets of $\mathbf{R}$. Take $b \in A(\mathbf{R})$ and $z \in A^{-1}(b)$. Set $b^{\rho}=A^{\rho}(z)$; we have $b^{\rho} \rightarrow b$ as $\rho \rightarrow 0$. Using (i) and the integration-by-parts formula for the Stieltjes integral, we get

$$
\begin{aligned}
\left|\widetilde{A}_{\theta}^{\rho}(b)-\widetilde{A}_{\theta}(b)\right| & \leq\left|\widetilde{A}_{\theta}^{\rho}(b)-\widetilde{A}_{\theta}^{\rho}\left(b^{\rho}\right)\right|+\left|\widetilde{A}_{\theta}^{\rho}\left(b^{\rho}\right)-\widetilde{A}_{\theta}(b)\right| \\
& \leq\|\theta\|_{L^{\infty}}\left|b-b^{\rho}\right|+\left|\int_{0}^{z} \theta(s) d\left(A^{\rho}(s)-A(s)\right)\right| \\
& \leq 2\|\theta\|_{L^{\infty}}\left|b-b^{\rho}\right|+\left|\int_{0}^{z}\left(A^{\rho}(s)-A(s)\right) \theta^{\prime}(s) d s\right| .
\end{aligned}
$$

The right-hand side converges to zero as $\rho \rightarrow 0$. Thus the claim follows. 
We have now came to the definition of an entropy solution. Here and in the sequel, $\mathbf{R}^{ \pm}$denote $\{k \in \mathbf{R} \mid \pm k \geq 0\}$, respectively.

Definition 2.2 (entropy solution). An entropy solution of the initial-boundary value problem (1) is a measurable function $u: Q_{T} \rightarrow \mathbf{R}$ satisfying

(D.1) $u \in L^{\infty}(Q)$ and $w=A(u) \in L^{p}\left(0, T ; W_{0}^{1, p}(\Omega)\right)$;

(D.2) for all $\psi \in \mathcal{D}([0, T) \times \Omega)$,

$$
\begin{gathered}
\int_{Q}\left(u \partial_{t} \psi+\mathfrak{f}(u) \cdot \nabla \psi-k(\nabla w) \nabla w \cdot \nabla \psi\right) d x d t \\
+\int_{\Omega} u_{0} \psi(0, \cdot) d x+\int_{Q} \mathcal{S} \psi d x d t=0
\end{gathered}
$$

(D.3) for all pairs $(c, \psi) \in \mathbf{R}^{ \pm} \times \mathcal{D}([0, T) \times \bar{\Omega}), \psi \geq 0$, and also for all pairs $(c, \psi) \in \mathbf{R} \times \mathcal{D}([0, T) \times \Omega), \psi \geq 0$,

$$
\begin{gathered}
\int_{Q}\left(\eta_{c}^{ \pm}(u) \partial_{t} \psi+\mathfrak{q}_{c}^{ \pm}(u) \cdot \nabla \psi-k(\nabla w) \nabla \widetilde{A}_{\left(\eta_{c}^{ \pm}\right)^{\prime}}(w) \cdot \nabla \psi\right) d x d t \\
+\int_{\Omega} \eta_{c}^{ \pm}\left(u_{0}\right) \psi(0, \cdot) d x+\int_{Q}\left(\eta_{c}^{ \pm}\right)^{\prime}(u) \mathcal{S} \psi d x d t \geq 0 .
\end{gathered}
$$

For the convergence proof we need the notion of entropy double-process solutions; we adapt this notion from [48, 31, 54, 49, where entropy process solutions have been introduced for hyperbolic problems and degenerate parabolic problems with linear diffusion. This definition is based upon the so-called "nonlinear $L^{\infty}$ weak-^ convergence" property, which is well-known in the equivalent framework of measurevalued solutions developed earlier by Tartar and DiPerna:

$$
\mid \begin{aligned}
& \text { each sequence }\left(u_{\rho}\right) \text { bounded in } L^{\infty}(Q) \text { admits a subsequence such that } \\
& \forall F \in C(\mathbf{R}), \quad F\left(u_{\rho}(\cdot, \cdot)\right) \rightarrow \int_{0}^{1} F(\mu(\cdot, \cdot, \alpha)) d \alpha \text { in } L^{\infty}(Q) \text { weak- },
\end{aligned}
$$

where the function $\mu \in L^{\infty}(Q \times(0,1))$ is referred to as the "process function"; it is related to the distribution function of the Young measure. As usual, in (5) and elsewhere we do not bother to (re)label sequences.

We remark that the reason for introducing in the definition below two different process functions $\mu, \mu^{*}$, both corresponding to the single unknown function $u$, is that it permits us to handle the double approximation of $u$ by pairs $u^{\mathfrak{M}}, u^{\mathfrak{M}^{*}}$ in the framework of DDFV schemes (see Section 3 ).

Definition 2.3 (entropy double-process solution). A triplet $\left(\mu, \mu^{*}, w\right)$ of measurable functions, with $\mu, \mu^{*}: Q \times(0,1) \rightarrow \mathbf{R}$ and $w: Q \rightarrow \mathbf{R}$, is called an entropy double-process solution of the initial-boundary value problem (1) if the following conditions are met:

(D'.1) $\mu, \mu^{*} \in L^{\infty}(Q \times(0,1)), w \in L^{p}\left(0, T ; W_{0}^{1, p}(\Omega)\right)$, and

$$
A(\mu(t, x, \alpha)) \equiv w(t, x) \equiv A\left(\mu^{*}(t, x, \alpha)\right),
$$

for a.e. $(t, x, \alpha) \in Q \times(0,1)$. 
(D'.2) For all $\psi \in \mathcal{D}([0, T) \times \Omega)$,

$$
\begin{gathered}
\int_{0}^{1} \int_{Q}\left(\frac{1}{d}\left(\mu+(d-1) \mu^{*}\right) \partial_{t} \psi+\frac{1}{d}\left(\mathfrak{f}(\mu)+(d-1) \mathfrak{f}\left(\mu^{*}\right)\right) \cdot \nabla \psi\right) d x d t d \alpha \\
-\int_{Q} k(\nabla w) \nabla w \cdot \nabla \psi d x d t+\int_{\Omega} u_{0} \psi(0, \cdot) d x+\int_{Q} \mathcal{S} \psi d x d t=0 .
\end{gathered}
$$

(D'.3) For all pairs $(c, \psi) \in \mathbf{R}^{ \pm} \times \mathcal{D}([0, T) \times \bar{\Omega}), \psi \geq 0$, and also for all pairs $(c, \psi) \in \mathbf{R} \times \mathcal{D}([0, T) \times \Omega), \psi \geq 0$,

$$
\begin{gathered}
\int_{0}^{1} \int_{Q}\left(\frac{1}{d}\left(\eta_{c}^{ \pm}(\mu)+(d-1) \eta_{c}^{ \pm}\left(\mu^{*}\right)\right) \partial_{t} \psi+\frac{1}{d}\left(\mathfrak{q}_{c}^{ \pm}(\mu)+(d-1) \mathfrak{q}_{c}^{ \pm}\left(\mu^{*}\right)\right) \cdot \nabla \psi\right) d x d t d \alpha \\
-\int_{Q} k(\nabla w) \nabla \widetilde{A}_{\left(\eta_{c}^{ \pm}\right)^{\prime}}(w) \cdot \nabla \psi d x d t+\int_{\Omega} \eta_{c}^{ \pm}\left(u_{0}\right) \psi(0, \cdot) d x \\
+\int_{0}^{1} \int_{Q} \frac{1}{d}\left(\left(\eta_{c}^{ \pm}\right)^{\prime}(\mu)+(d-1)\left(\eta_{c}^{ \pm}\right)^{\prime}\left(\mu^{*}\right)\right) \mathcal{\delta} \psi d x d t d \alpha \geq 0 .
\end{gathered}
$$

Remark 2.1. Since $\nabla w=0$ a.e. on $\{(t, x) \in Q) \mid w(t, x)=A(c)\}$ for any $c \in \mathbf{R}$, the term $k(\nabla w) \nabla \widetilde{A}_{\left(\eta_{c}^{ \pm}\right)^{\prime}}(w)$ in the above definitions can be rewritten as

$$
\left(\eta_{c}^{ \pm}\right)^{\prime}(z) \mathfrak{a}(\nabla w) \text { for any } z \in A^{-1}(w), \text { and also as } \operatorname{sign}^{ \pm}(w-A(c)) \mathfrak{a}(\nabla w) .
$$

The form used in (D.3) and (D'.3) is convenient for expressing the approximate entropy inequalities at the discrete level; the equivalent form (6) is used in the uniqueness proof. Both forms are exploited in the existence proof below.

Remark 2.2. Let $u$ be an entropy solution of (1). Then the triplet $\left(\mu, \mu^{*}, w\right)$ defined by

$$
\begin{aligned}
& \mu(t, x, \alpha)=\mu^{*}(t, x, \alpha)=u(t, x) \text { for a.e. }(t, x, \alpha) \in Q \times(0,1), \\
& w(t, x)=A(u(t, x)) \text { for a.e. }(t, x) \in Q .
\end{aligned}
$$

is an entropy double-process solution of (1).

Conversely, if $\left(\mu, \mu^{*}, w\right)$ is an entropy double-process solution of (1) for which $\mu(t, x, \alpha)=\mu^{*}(t, x, \alpha)=u(t, x)$ a.e. on $Q \times(0,1)$ for some function $u: Q \rightarrow \mathbf{R}$, then this $u$ is an entropy solution of (1).

Note that in Definition 2.2. we have only considered $\alpha$-independent data $u_{0}, f$. In this case, the notion of entropy double-process solution is just a technical tool that permits to bypass the lack of strong compactness of sequences of approximate solutions. As a first illustration of this, we pass to the limit in vanishing viscosity approximations (without $B V$ estimates) to prove the existence of an entropy doubleprocess solution such that $\mu \equiv \mu^{*}$.

Theorem 2.1. Under the assumptions stated in Section 1, there exists an entropy double-process solution to the initial-boundary value problem (11) for which $\mu \equiv \mu^{*}$.

Notice that the above result holds for any Lipschitz domain $\Omega$ in any space dimension. In passing, we also mention that the existence result of Theorem 2.1 has recently been generalized by Ouaro and the authors 9 to the case of a triply nonlinear degenerate diffusion equation. 
Proof. The proof is divided into several steps.

(i) We approximate problem (11) by regular problems (11) $\rho$ with $\mathfrak{f}, A$ replaced by $\mathfrak{f}_{\rho}, A_{\rho}$ such that $\mathfrak{f}_{\rho}, A_{\rho},\left[A_{\rho}\right]^{-1}$ are Lipschitz continuous on $\mathbf{R}$ and $\mathfrak{f}_{\rho}, A_{\rho}$ converge to $\mathfrak{f}, A$, respectively, uniformly on compacts sets as $\rho \rightarrow 0$.

Using classical techniques (cf. Alt, Luckhaus [4 and Lions 64]), we can show that there exists a weak solution $u_{\rho} \in L^{p}\left(0, T ; W_{0}^{1, p}(\Omega)\right)$ to problem (1) $\rho$ in the following sense:

$$
\left\{\begin{array}{c}
\partial_{t} u_{\rho}+\operatorname{div} \mathfrak{f}_{\rho}\left(u_{\rho}\right)=\operatorname{div} \mathfrak{a}\left(\nabla A_{\rho}\left(u_{\rho}\right)\right)+\mathcal{S} \\
\text { in } L^{p^{\prime}}\left(0, T ; W^{-1, p^{\prime}}(\Omega)\right)+L^{1}(Q),\left.\quad u_{\rho}\right|_{t=0}=u_{0} .
\end{array}\right.
$$

Moreover, since $\mathfrak{f}_{\rho} \circ A_{\rho}^{-1}$ is Lipschitz continuous, the $L^{1}$ contraction property and comparison principle for weak solutions can be verified. It can be obtained either by the technique of Otto [70] (doubling the time variable) or using the theory of integral solutions and nonlinear semigroup methods, consult for example 30. Besides, $u_{\rho}$ verifies the entropy formulation of Definition 2.2 with fluxes $\mathfrak{f}_{\rho}, A_{\rho}$, where $\eta_{c}^{ \pm}$can be replaced by regular "boundary" entropies $\eta_{c, \varepsilon}^{ \pm}$, whenever we prefer to do so.

(ii) We claim that the following quantities are uniformly bounded in $\rho$ :

- $\left\|u_{\rho}\right\|_{L^{\infty}(\Omega)}$ and $\left\|A_{\rho}\left(u_{\rho}\right)\right\|_{L^{p}\left(0, T ; W_{0}^{1, p}(\Omega)\right)}$;

- space translates of $A_{\rho}\left(u_{\rho}\right)$ in $L^{1}(Q)$ (consequence of previous estimate);

- time translates of $A_{\rho}\left(u_{\rho}\right)$ in $L^{1}(Q)$.

Indeed, for the first point consider the function

$$
M(t)=\left\|u_{0}\right\|_{L^{\infty}(\Omega)}+\int_{0}^{t}\|\mathcal{S}(\tau, \cdot)\|_{L^{\infty}(\Omega)} d \tau,
$$

which is a solution of (11) $\rho$ with $x$-constant data $\left\|u_{0}\right\|_{L^{\infty}(\Omega)},\|\mathcal{S}(t, \cdot)\|_{L^{\infty}(\Omega)}$. The comparison principle mentioned in (i) ensures that a.e. on $Q$,

$$
-M(T) \leq-M(t) \leq u_{\rho}(t, x) \leq M(t) \leq M(T) .
$$

Next, we employ $A_{\rho}\left(u_{\rho}\right)$ as a test function in (7). The product between $\partial_{t} u_{\rho}$ and $A_{\rho}\left(u_{\rho}\right)$ is handled using the usual chain rule argument (see, e.g., 4, 70, 30]), where the relevant duality is between the space $E:=L^{p}\left(0, T ; W_{0}^{1, p}(\Omega)\right) \cap L^{\infty}(Q)$ and the space $L^{p^{\prime}}\left(0, T ; W^{-1, p^{\prime}}(\Omega)\right)+L^{1}(Q) \subset E^{*}$. Here we are also exploiting the $L^{\infty}$ bound on $\mathfrak{f}_{\rho}\left(u_{\rho}\right)$ in a straightforward fashion to treat the term $\mathfrak{f}_{\rho}\left(u_{\rho}\right) \cdot \nabla A_{\rho}\left(u_{\rho}\right)$; but notice that using the Green-Gauss trick (16) below, we can supply a finer analysis of this term.

For the third bullet point, we first use (17) to get, for a.e. $t, t+\Delta \in(0, T)$,

$$
\int_{\Omega}\left(u_{\rho}(t+\Delta)-u_{\rho}(t)\right) \xi=\int_{t}^{t+\Delta} \int_{\Omega}\left[\left(-\mathfrak{f}_{\rho}\left(u_{\rho}\right)+\mathfrak{a}\left(\nabla A_{\rho}\left(u_{\rho}\right)\right)\right) \cdot \nabla \xi+\mathcal{S} \xi\right]
$$

for all $\xi \in W_{0}^{1, p}(\Omega) \cap L^{\infty}(\Omega)$. Taking $\xi=A_{\rho}\left(u_{\rho}(t+\Delta)\right)-A_{\rho}\left(u_{\rho}(t)\right)$ and integrating in $t$, using the two previously obtained estimates, we deduce that

$$
\iint_{Q}\left|u_{\rho}(t+\Delta)-u_{\rho}(t)\right|\left|A_{\rho}\left(u_{\rho}(t+\Delta)\right)-A_{\rho}\left(u_{\rho}(t)\right)\right| \leq \text { Const }|\Delta| .
$$

Now, let $\pi$ be a (common for all $\rho$ ) concave modulus of continuity for $A_{\rho}$ on $[-M(T), M(T)], \Pi$ be its inverse, and set $\tilde{\Pi}(r)=r \Pi(r)$. Let $\tilde{\pi}$ be the inverse 
of $\tilde{\Pi}$. Note that $\tilde{\pi}$ is concave, continuous, and $\tilde{\pi}(0)=0$. Set $v(t, x)=u_{\rho}(t+\Delta, x)$ and $y(t, x)=u_{\rho}(t, x)$. We have

$$
\begin{aligned}
\int_{Q}\left|A_{\rho}(v)-A_{\rho}(y)\right| & =\int_{Q} \tilde{\pi}\left(\tilde{\Pi}\left(\left|A_{\rho}(v)-A_{\rho}(y)\right|\right)\right) \\
& \leq|Q| \tilde{\pi}\left(\frac{1}{|Q|} \int_{Q} \tilde{\Pi}\left(\left|A_{\rho}(v)-A_{\rho}(y)\right|\right)\right) .
\end{aligned}
$$

Since $\left|A_{\rho}(v)-A_{\rho}(y)\right| \leq \pi(|v-y|)$, we have $\Pi\left(\left|A_{\rho}(v)-A_{\rho}(y)\right|\right) \leq|v-y|$ and

$$
\begin{aligned}
\tilde{\Pi}\left(\left|A_{\rho}(v)-A_{\rho}(y)\right|\right) & =\Pi\left(\left|A_{\rho}(v)-A_{\rho}(y)\right|\right)\left|A_{\rho}(v)-A_{\rho}(y)\right| \\
& \leq|v-y|\left|A_{\rho}(v)-A_{\rho}(y)\right| .
\end{aligned}
$$

Therefore, estimate (8) implies

$$
\begin{aligned}
\int_{Q} \mid & A_{\rho}\left(u_{\rho}(t+\Delta, x)\right)-A_{\rho}\left(u_{\rho}(t, x)\right) \mid \\
& \leq|Q| \tilde{\pi}\left(\frac{1}{|Q|} \int_{Q}|v-y|\left|A_{\rho}(v)-A_{\rho}(y)\right|\right) \\
& =|Q| \tilde{\pi}\left(\frac{1}{|Q|} J(\Delta)\right) \leq C \tilde{\pi}(C \Delta)=: \omega_{A}(\Delta),
\end{aligned}
$$

where $\omega_{A} \in C\left(\mathbf{R}^{+}, \mathbf{R}^{+}\right), \omega_{A}(0)=0$.

(iii) Thanks to the estimates in (ii) and standard compactness results, there exists a (not labelled) sequence $\rho \rightarrow 0$ such that

- $w_{\rho}=A_{\rho}\left(u_{\rho}\right)$ converges strongly in $L^{1}(Q)$ and pointwise a.e. on $Q$;

- $\nabla w_{\rho}$ converges weakly in $L^{p}(Q)$;

- $\mathfrak{a}\left(\nabla w_{\rho}\right)$ converges weakly in $L^{p^{\prime}}(Q)$ to some limit $\chi$;

- $u_{\rho}$ converges to $\mu: Q \times(0,1) \in \mathbf{R}$ in the sense of (5).

Let us introduce the function

$$
u(t, x)=\int_{0}^{1} \mu(t, x, \alpha) d \alpha, \quad \text { for a.e. }(t, x) \in Q .
$$

Thanks to the convergence of $A_{\rho}$ to $A$, we can identify the limit of $w_{\rho}(\cdot, \cdot)$ with $\int_{0}^{1} A(\mu(\cdot, \cdot, \alpha)) d \alpha$. Moreover, since $w_{\rho}$ is converging strongly, $A(\mu(\cdot, \cdot, \alpha))$ is actually independent of $\alpha \in(0,1)$ and equals $A(u(\cdot, \cdot))$. Using distributional derivatives, we also identify the limit of $\nabla w_{\rho}$ with $\nabla A(u)$.

(iv) We have now come to the main step of the proof, namely to improve the weak convergence of $\nabla A_{\rho}\left(u_{\rho}\right)$ to strong convergence, and to identify the weak limit of $\mathfrak{a}\left(\nabla A_{\rho}\left(u_{\rho}\right)\right)$ with $\mathfrak{a}(\nabla A(u))$, where $u$ is defined in (10); of course, the chief difficulty comes from the lack of strong convergence of $u_{\rho}$.

We begin by specifying the test function in (7) as $w_{\rho} \zeta$, yielding

$$
\underbrace{\int_{0}^{T}\left\langle\partial_{t} u_{\rho}, w_{\rho} \zeta\right\rangle}_{I_{1, \rho}}-\underbrace{\int_{Q} \mathfrak{f}_{\rho}\left(u_{\rho}\right) \cdot \nabla w_{\rho} \zeta}_{I_{2, \rho}}+\int_{Q} \mathfrak{a}\left(\nabla w_{\rho}\right) \cdot \nabla w_{\rho} \zeta-\underbrace{\int_{Q} \mathcal{S} w_{\rho} \zeta}_{I_{3, \rho}}=0,
$$

where $w_{\rho}=A_{\rho}\left(u_{\rho}\right)$ and $\zeta \in \mathcal{D}([0, T))$ is nonincreasing with $\zeta(0)=1$. Next, we pass to the limit into the weak formulation (7), obtaining

$$
\left\{\begin{array}{l}
\partial_{t} u+\operatorname{div} \int_{0}^{1} \mathfrak{f}(\mu) d \alpha=\operatorname{div} \chi+\mathcal{S} \\
\quad \text { in } L^{p^{\prime}}\left(0, T ; W^{-1, p^{\prime}}(\Omega)\right)+L^{1}(Q),\left.\quad u_{\rho}\right|_{t=0}=u_{0} .
\end{array}\right.
$$


In (12), we take $w \zeta$ as test function, where $w=A(u), u$ is defined in (10), and $\zeta$ is as specified above. The result is

$$
\underbrace{\int_{0}^{T}\left\langle\partial_{t} u, w \zeta\right\rangle}_{I_{1}}-\underbrace{\int_{Q} \int_{0}^{1} \mathfrak{f}(\mu) \cdot \nabla w \zeta}_{I_{2}}+\int_{Q} \chi \cdot \nabla A(u) \zeta-\underbrace{\int_{Q} \mathcal{S} w \zeta}_{I_{3}}=0 .
$$

In order to later use the Minty-Browder trick, we shall combine (13) and the " $\rho \rightarrow 0$ " limit of (11) to conclude the validity of the following inequality:

$$
\int_{Q} \chi \cdot \nabla A(u) \geq \liminf _{\rho \rightarrow 0} \int_{Q} \mathfrak{a}\left(\nabla w_{\rho}\right) \cdot \nabla w_{\rho}
$$

A crucial role is played by the following calculation, which reveals that the lack of strong convergence of $\mathfrak{f}_{\rho}\left(u_{\rho}\right)$ is not an obstacle. Indeed, a componentwise application of Lemma 2.1 (i) yields the existence of a Lipschitz continuous vectorvalued function $\widetilde{A}_{\mathrm{f}}$ such that

$$
\int_{0}^{z} \mathfrak{f}(s) d A(s)=\widetilde{A}_{\mathfrak{f}}(A(z)) .
$$

Hence, by the chain rule and the Green-Gauss formula, we can calculate as follows:

$$
\begin{aligned}
\int_{Q} \int_{0}^{1} \mathfrak{f}(\mu) \cdot \nabla A(u) & =\int_{Q} \int_{0}^{1} \mathfrak{f}(\mu) \cdot \nabla A(\mu)=\int_{0}^{1} \int_{0}^{T} \int_{\Omega} \operatorname{div} \widetilde{A}_{\mathfrak{f}}(A(\mu)) \\
& =\int_{0}^{T} \int_{\partial \Omega} \widetilde{A}_{\mathfrak{f}}(A(u)) \cdot n=0,
\end{aligned}
$$

because for a.e. $\alpha \in(0,1)$,

$$
A(\mu(\cdot, \cdot, \alpha))=A(u(\cdot, \cdot)) \in L^{p}\left(0, T ; W_{0}^{1, p}(\Omega)\right)
$$

By similar (simpler) arguments and $u_{\rho} \in L^{p}\left(0, T ; W_{0}^{1, p}(\Omega)\right)$, we also have

$$
\int_{Q} \mathfrak{f}_{\rho}\left(u_{\rho}\right) \cdot \nabla A_{\rho}\left(u_{\rho}\right)=\int_{0}^{T} \int_{\Omega} \operatorname{div}\left(\int_{0}^{u_{\rho}} \mathfrak{f}_{\rho}(s) d A_{\rho}(s)\right)=0 .
$$

Consequently, we can make $I_{2}$ and $I_{2, \rho}$ (for each $\rho>0$ ) vanish.

Next, let us prove that $I_{1} \leq \lim _{\rho \rightarrow 0} I_{1, \rho}$. As above, the duality products $\left\langle\partial_{t} u_{\rho}, A_{\rho}\left(u_{\rho}\right)\right\rangle,\left\langle\partial_{t} u, A(u)\right\rangle$ are treated via the chain rule argument (cf. [4). Set $B(z)=\int_{0}^{z} A(s) d s, B_{\rho}(z)=\int_{0}^{z} A_{\rho}(s) d s$, and note that these functions are convex. Also, $B_{\rho} \rightarrow B$ uniformly on compact subsets of $\mathbf{R}$. With the help of Jensen's 
inequality,

$$
\begin{aligned}
I_{1} & =\int_{0}^{T}\left\langle\partial_{t} u, A(u) \zeta\right\rangle=-\int_{Q} B(u) \zeta^{\prime}-\int_{\Omega} B\left(u_{0}\right) \\
& =\int_{Q} B\left(\int_{0}^{1} \mu(t, x, \alpha) d \alpha\right)\left(-\zeta^{\prime}\right)-\int_{\Omega} B\left(u_{0}\right) \\
& \leq \int_{Q} \int_{0}^{1} B(\mu(t, x, \alpha)) d \alpha\left(-\zeta^{\prime}\right)-\int_{\Omega} B\left(u_{0}\right) \\
& =\lim _{\rho \rightarrow 0}\left(-\int_{Q} B_{\rho}\left(u_{\rho}\right) \zeta^{\prime}-\int_{\Omega} B_{\rho}\left(u_{0}\right)\right) \\
& =\lim _{\rho \rightarrow 0} \int_{0}^{T}\left\langle\partial_{t} u_{\rho}, A_{\rho}\left(u_{\rho}\right) \zeta\right\rangle=\lim _{\rho \rightarrow 0} I_{1, \rho} .
\end{aligned}
$$

Finally, it is clear that $I_{3, \rho} \rightarrow I_{3}$ as $\rho \rightarrow 0$. Letting $\zeta$ tend to $\mathbb{1}_{[0, T)}$, the desired inequality (14) follows from subtracting the " $\rho \rightarrow 0$ " limit of (11) from (13) and the above calculations.

Starting off from (14), we can use the Minty-Browder trick (see, for example, [64, 24, 4, 21] and the proof of Theorem 7.1 in Section 7) to deduce that

$$
\mathfrak{a}\left(\nabla w_{\rho}\right)-\mathfrak{a}(\nabla A(u)) \rightarrow 0 \quad \text { weakly in } L^{p^{\prime}}(Q) \text { as } \rho \rightarrow 0 .
$$

Thus $\chi=\mathfrak{a}(\nabla A(u))$. Simultaneously, from the strict monotonicity of $\mathfrak{a}(\cdot)$ we deduce that, firstly, the convergence in (17) also takes place a.e. in $Q$; secondly, that (14) actually holds with an equality sign. Next, we consider the functions $g_{\rho}:=\mathfrak{a}\left(\nabla w_{\rho}\right) \cdot \nabla w_{\rho} \geq 0$ and $g:=\mathfrak{a}(\nabla A(u)) \cdot \nabla A(u) \geq 0$, and observe that

$$
g_{\rho} \rightarrow g \text { a.e. in } Q, \quad \int_{Q} g_{\rho} \rightarrow \int_{Q} g \quad \text { as } \rho \rightarrow 0 .
$$

Hence, we deduce that a subsequence of $\left(g_{\rho}\right)_{\rho}$ converges to $g$ strongly in $L^{1}(Q)$, cf. [24, 21, Lemma 5], 42, Lemma 8.4]. Due to the coercivity of $\mathfrak{a}(\cdot),\left(\left|\nabla w_{\rho}\right|^{p}\right)_{\rho}$ is equi-integrable, so the Vitali theorem yields the strong $L^{p}$ convergence of $\nabla w_{\rho}$, along a subsequence if necessary, to a limit already identified as $\nabla w, w=A(u)$.

(v) By (12), we readily conclude that $(\mu, \mu, w)$ verifies (D'.2). Now we can pass to the limit in the entropy inequalities corresponding to (1) $\rho$ and deduce (D'.3).

Let us first show that $\nabla \widetilde{A}_{\left(\eta_{c, \varepsilon}^{ \pm}\right)^{\prime}}^{\rho}\left(w_{\rho}\right)$ converges weakly to $\nabla \widetilde{A}_{\left(\eta_{c, \varepsilon}^{ \pm}\right)^{\prime}}(w)$ in $L^{p}(Q)$. By Lemma 2.1 (i), $A_{\left(\eta_{c, \varepsilon}^{ \pm}\right)^{\prime}}^{\rho}(\cdot)$ are uniformly Lipshitz continuous functions. Thus $\nabla \widetilde{A}_{\left(\eta_{c, \varepsilon}^{ \pm}\right)^{\prime}}^{\rho}\left(w_{\rho}\right)$ are uniformly bounded and weakly compact in $L^{p}(Q)$. Moreover, $\widetilde{A}_{\left(\eta_{c, \varepsilon}^{ \pm}\right)^{\prime}}^{\rho}\left(w_{\rho}\right)$ converges to $\widetilde{A}_{\left(\eta_{c, \varepsilon}^{ \pm}\right)^{\prime}}(w)$ by Lemma2.1 (ii) and because of the pointwise convergence of $w_{\rho}$ to $w$. Using the distributional convergence, we eventually work out our claim.

Now note that if $p>2$, then $k$ is continuous. By the last result of (iv), we can assume without loss of generality that $k\left(\nabla w_{\rho}\right)$ converges to $k(\nabla w)$ a.e. in $Q$. Moreover, $\left(k\left(\nabla w_{\rho}\right)\right)$ is bounded in $L^{\frac{p}{p-2}}(Q)$, since $\left(\nabla w_{\rho}\right)$ is bounded in $L^{p}(Q)$. Applying the Egorov theorem and Hölder's inequality with exponents $p^{\prime}, p$ in the product $\left(k\left(\nabla w_{\rho}\right) \nabla \psi\right) \cdot \nabla \widetilde{A}_{\left(\eta_{c, \varepsilon}^{ \pm}\right)^{\prime}}\left(w_{\rho}\right)$, we deduce that

$$
\lim _{\rho \rightarrow 0} \int_{Q} k\left(\nabla w_{\rho}\right) \nabla \widetilde{A}_{\left(\eta_{c, \varepsilon}^{ \pm}\right)^{\prime}}\left(w_{\rho}\right) \cdot \nabla \psi=\int_{Q} k(\nabla w) \nabla \widetilde{A}_{\left(\eta_{c, \varepsilon}^{ \pm}\right)^{\prime}}(w) \cdot \nabla \psi .
$$


If $p \leq 2$, we fix a small $\delta>0$ and truncate $k(\cdot)$ in the $\delta$-neigbourhood of the origin (if $k(\cdot)$ is replaced by $k_{\delta}(\cdot)=\min \left\{k(\cdot), \min _{|\xi| \leq \delta} k(\xi)\right\}$, the argument used for $p>2$ applies), and we analyze separately the set $\left\{(t, x)|| \nabla w^{\rho}(t, x) \mid<\delta\right\}$. On this set,

$$
k\left(\nabla w_{\rho}\right)\left|\nabla \widetilde{A}_{\left(\eta_{c, \varepsilon}^{ \pm}\right)^{\prime}}\left(w_{\rho}\right)\right| \leq C\left\|\left(\eta_{c, \varepsilon}^{ \pm}\right)^{\prime}\right\|_{\infty}\left|\nabla w_{\rho}\right|^{p-1} \leq \text { Const } \delta^{p-1},
$$

uniformly in $\rho$. To conclude that (18) still holds, we first pass to the limit as $\rho \rightarrow 0$ for a fixed $\delta>0$, and then send $\delta \rightarrow 0$.

Let us take regular "boundary" entropy pairs $\eta_{c, \varepsilon}^{ \pm}$such that $\left(\eta_{c, \varepsilon}^{ \pm}\right)^{\prime}$ approximate $\left(\eta_{c}^{ \pm}\right)^{\prime}$ (extended by zero at the point $c$, by our convention), pointwise a.e. in $\mathbf{R}$ as $\varepsilon \rightarrow 0$. We use (18) to pass to the limit in the entropy inequality corresponding to (11) $\rho$. We pass to the limit in the remaining terms in this entropy inequality

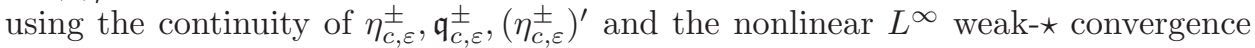
property (5). Finally, we pass to the limit as $\varepsilon \rightarrow 0$, rewriting $\nabla \widetilde{A}_{\left(\eta_{c, \varepsilon}^{ \pm}\right)^{\prime}}(w)$ as $\left(\eta_{c, \varepsilon}^{ \pm}\right)^{\prime}(u) \nabla w$ (consult Remark 2.1) and using the Lebesgue dominated convergence theorem and the pointwise convergences of $\eta_{c, \varepsilon}^{ \pm}, \mathfrak{q}_{c, \varepsilon}^{ \pm},\left(\eta_{c, \varepsilon}^{ \pm}\right)^{\prime}$. The passage to the limit in the weak formulation is similar.

(vi) We conclude that $\left(\mu, \mu^{*}, A(u)\right)$ is an entropy double-process solution of (1) such that $\mu^{*}=\mu$.

Given Theorem 2.1, the uniqueness of an entropy double-process solution can be established using Kruzhkov's method, along the lines of Carrillo [29].

Theorem 2.2. Suppose the assumptions stated in Section 1 hold. Let $\left(\mu, \mu^{*}, w\right)$ be an entropy double-process solution of the initial-boundary value problem (1). Then it is unique. Moreover, there exists a function $u \in L^{\infty}(Q)$ such that

$$
\mu(t, x, \alpha)=u(t, x)=\mu^{*}(t, x, \alpha) \text { for a.e. }(t, x, \alpha) \in Q \times(0,1) .
$$

We refer to Appendix A for a sketch of the proof.

Theorems 2.1 and 2.2 as well as the arguments of Appendix A imply

Corollary 2.1 (well-posedness). Under the assumptions stated in Section 1, there exists a unique entropy solution of the initial-boundary value problem (1). Let $u$ and $v$ be two entropy solutions of (1) with initial data $\left.u\right|_{t=0}=u_{0} \in L^{\infty}(\Omega)$ and $\left.v\right|_{t=0}=v_{0} \in L^{\infty}(\Omega)$ and source terms $\mathcal{S}$ and $\mathcal{T}$ of the kind (2), respectively. For a.e. $t \in(0, T)$, we have

$$
\int_{\Omega}(u(t, x)-v(t, x))^{+} d x \leq \int_{\Omega}\left(u_{0}-v_{0}\right)^{+} d x+\int_{0}^{t} \int_{\Omega}(\mathcal{S}-\mathcal{T})^{+} .
$$

Consequently, if $u_{0} \leq v_{0}$ a.e. in $\Omega$ and $\mathcal{S} \leq \mathcal{T}$ a.e. on $Q$, then $u \leq v$ a.e. in $Q$. Finally, if $u_{0}=v_{0}$ a.e. in $\Omega$ and $\mathcal{S}=\mathcal{T}$ a.e. on $Q$, then $u=v$ a.e. in $Q$.

The upcoming sections are concerned with the construction of finite volume schemes for which the corresponding discrete solutions converge to the unique entropy solution of (11) as the discretization parameter (mesh size) tends to zero. The convergence proof will attempt to mimic the proof of Theorem 2.1.

\section{Discrete Duality finite volume (DDFV) Schemes}

Let $\Omega$ be a polygonal (respectively, polyhedral) open bounded subset of $\mathbf{R}^{d}$, $d=2$ (respectively, $d=3$ ). In what follows, we introduce most of the notation related to DDFV schemes; each piece of new notation is given in italic script. 


\subsection{Construction of "double" conformal meshes.}

- A partition of $\Omega$ is a finite set of disjoint open polygonal (respectively, polyhedral) subsets of $\Omega$ such that $\Omega$ is contained in their union, up to a set of zero $d$-dimensional measure.

Following Hermeline [57, Domelevo, Omnès 41 and Andreianov, Boyer, Hubert [1], we consider a DDFV mesh which is a triple $\mathfrak{T}=\left(\overline{\mathfrak{M}}, \overline{\mathfrak{M}^{*}}, \mathfrak{S}\right)$ described below.

- We let $\mathfrak{M}$ be a partition of $\Omega$ into triangles (respectively, tetrahedra); a more general case is discussed in Section 团. We assume that the mesh satisfies the Delaunay condition (see, e.g., 48]); for simplicity of the representation, the reader may assume that each triangle (respectively, tetrahedron) contains the centre if its circumscribed circle (respectively, ball). We assume in addition

$$
\begin{aligned}
& \text { if } d=3 \text {, each face of each tetrahedron of } \mathfrak{M} \\
& \text { contains the centre of its circumscribed circle. }
\end{aligned}
$$

Although the definition of the scheme does not require condition (19) (see Remark 3.1 below), we do need this condition in order to deduce the discrete entropy inequalities and to prove that the scheme converges.

Each control volume $K \in \mathfrak{M}$ is supplied with a centre $x_{K}$ that we choose to be the centre of the circle (respectively, ball) circumscribed around $K$. We call $\partial \mathfrak{M}$ the set of all edges (respectively, faces) of control volumes that are included in $\partial \Omega$. These edges (respectively, faces) are considered as boundary control volumes; for $K \in \partial \mathfrak{M}$, we choose the middle of $K$ (respectively, the centre of the circle circumscribed around $K$ ) for the centre $x_{K}$. We denote by $\overline{\mathfrak{M}}$ the union $\mathfrak{M} \cup \partial \mathfrak{M}$. We call vertex (of $\mathfrak{M}$ ) any vertex of any control volume $K \in \overline{\mathfrak{M}}$.

- (see Figure 1) We take $\overline{\mathfrak{M}^{*}}$ as the partition of $\Omega$ into dual control volumes $K^{*}$, supplied with dual centres $x_{K^{*}}$, such that $x_{K^{*}}$ is a vertex of $\mathfrak{M}$ and $K^{*}$ is the subset of points of $\Omega$ that are closen $\Omega$ to $x_{K^{*}}$ than to any other vertex of $\mathfrak{M}$. In other words, $\overline{\mathfrak{M}^{*}}$ is the Voronoï mesh constructed from the vertices of $\mathfrak{M}$. If $x_{K^{*}} \in \Omega$, we say that $K^{*}$ is a dual control volume and write $K^{*} \in \mathfrak{M}^{*}$; and if $x_{K^{*}} \in \partial \Omega$, we say that $K^{*}$ is a boundary dual control volume and write $K^{*} \in \partial \mathfrak{M}^{*}$. Thus $\overline{\mathfrak{M}^{*}}=\mathfrak{M}^{*} \cup \partial \mathfrak{M}^{*}$. We call dual vertex (of $\mathfrak{M}^{*}$ ) any vertex of any dual control volume $K^{*} \in \overline{\mathfrak{M}^{*}}$. Note that by the choice of $x_{K}$, the set of centres coincides with the set of dual vertices, and the set of vertices coincides with the set of dual centres. In other words, $\overline{\mathfrak{M}}$ and $\overline{\mathfrak{M}^{*}}$ are finite volume meshes that are dual each one to the other.

- We call neighbours of $K$, all control volumes $L \in \overline{\mathfrak{M}}$ such that $K$ and $L$ have a common edge (respectively, common face). The set of all neighbours of $K$ is denoted by $\mathcal{N}(K)$. Note that if $L \in \mathcal{N}(K)$, then $K \in \mathcal{N}(L)$; in this case we simply say that $K$ and $L$ are (a couple of) neighbours.

- (see Figures 1 and 2(b) If $K$ and $L$ are neighbours, we denote by $K L$ the interface $\partial K \cap \partial L$ between $K$ and $L$. The set of all interfaces is denoted by $\mathcal{E}$.

\footnotetext{
${ }^{1}$ In particular, in the two dimensional case we can partition $\Omega$ into polygons that admit a circumscribed circle. In the three dimensional case, we can partition $\Omega$ in polyhedra that have triangular faces and admit a circumscribed ball.

$2_{\text {in }}$ order to avoid pathological situations which could appear in non-convex domains, e.g., in domains with cracks, here the distance between two points $x, y$ of $\bar{\Omega}$ is understood as the length of the shortest path which connects $x$ with $y$ and which lies within $\bar{\Omega}$
} 


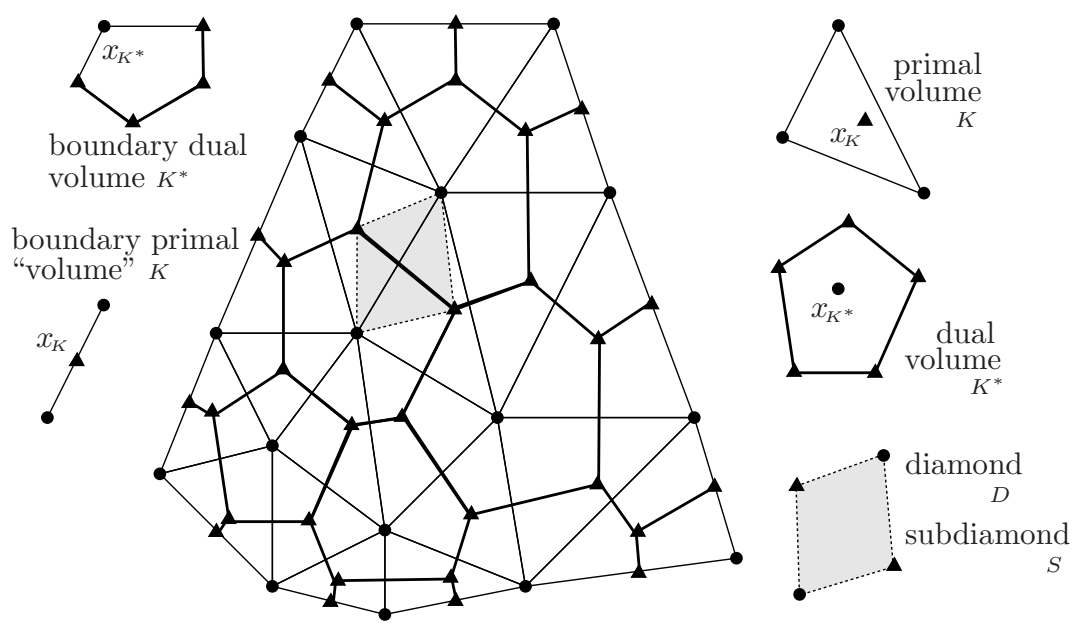

FIGURE 1. 2D primal and dual meshes; diamond (subdiamond).

- In the same way, we denote by $\mathcal{N}^{*}\left(K^{*}\right)$ the set of (dual) neighbours of a dual control volume $K^{*}$, and by $K^{*} \mid L^{*}$, the (dual) interface $\partial K^{*} \cap \partial L^{*}$ between dual neighbours $K^{*}$ and $L^{*}$. The set of all dual interfaces is denoted by $\mathcal{E}^{*}$.

- (see Figure 2) The meshes $\overline{\mathfrak{M}}$ and $\overline{\mathfrak{M}^{*}}$ induce partitions of $\Omega$ into diamonds and subdiamonds. Let us describe them separetely for $d=2$ and $d=3$.

For $d=2$ (see Figure 2(a) , if $K, L \in \overline{\mathfrak{M}}$ are neighbours, then there exists a unique couple of dual neighbours $\left\{K^{*}, L^{*}\right\}$ such that the interface $K \mid L$ is the segment with summits $x_{K^{*}}$ and $x_{L^{*}}$. Then the quadrilateral $D_{K^{*}, L^{*}}^{K, L}$ which is either the union (if $x_{K}, x_{L}$ lie on different sides from $K \mid L$ ) or the difference (if $x_{K}, x_{L}$ lie on the same side from $K(L)$ of the triangles $x_{K} x_{K^{*}} x_{L^{*}}, x_{L} x_{K^{*}} x_{L^{*}}$ is called a diamond; it is also unambiguously denoted by $D^{K, L}$.

For $d=2$, every diamond is also called a subdiamond; the subdiamond which coincides with a diamond $D^{K, L}$ is denoted by $S_{K^{*} *^{*}}^{K, L}$.

For $d=3$ (see Figure 2(b) $)$, if $K, L \in \overline{\mathfrak{M}}$ are neighbours, then there exists a unique triple of dual neighbours $\left\{K^{*}, L^{*}, M^{*}\right\}$ (which are neighbours pairwise) such that the interface $K \mid L$ is the triangle with summits $x_{K^{*}}, x_{L^{*}}$ and $x_{M^{*}}$. Then the polyhedron $D_{K^{*}, L^{*}, M^{*}}^{K, L}$ which is either the union (if $x_{K}, x_{L}$ lie on different sides from $K \mid L$ ) or the difference (if $x_{K}, x_{L}$ lie on the same side from $K \mid L$ ) of the pyramids $x_{K} x_{K^{*}} x_{L^{*}} x_{M^{*}}, x_{L} x_{K^{*}} x_{L^{*}} x_{M^{*}}$ is called a diamond; it is also unambiguously denoted by $D^{K, L}$. Each diamond is split into three subdiamonds; e.g., the subdiamond $S_{K^{*} L^{*}}^{K, L}$ is the convex hull of $x_{K}, x_{K^{*}}, x_{L}, x_{L^{*}}$.

We denote by $\mathfrak{D}, \mathfrak{S}$ the sets of all diamonds and the set of all subdiamonds, respectively. Generic elements of $\mathfrak{D}, \mathfrak{S}$ are denoted by $D, S$, respectively.

Remark 3.1. If we drop condition (19), the orthogonal projection of $x_{K}$ (which coincides with the projection of $x_{L}$ ) on $K \mid L$ may not be contained within $K \mid L$. To cope with this problem, one could consider subdiamonds of signed volume, not necessarily contained within the corresponding diamonds. Up to a permutation of the subscripts $K^{*}, L^{*}, M^{*}$, we have instead of the decomposition $D_{K^{*} L^{*}, M^{*}}^{K, L}=$ $S_{K^{*}, L^{*}}^{K, L} \cup S_{L^{*}, M^{*}}^{K, L} \cup S_{M^{*}, K^{*}}^{K, L}$, the decomposition $D_{K^{*}, L^{*}, M^{*}}^{K, L}=\left(S_{K^{*}, L^{*}}^{K, L} \cup S_{L^{*}, M^{*}}^{K, L}\right) \backslash S_{M^{*}, K^{*}}^{K, L}$; 
in this case the volume of $S_{M^{*}, K^{*}}^{K, L}$ will be taken with the sign "minus". Under this convention, Lemma 3.1 below holds true, so that formulas (22), (23)-(25) below still yield consistent discrete gradient and discrete divergence operators which enjoy the discrete duality property [7. But the discrete entropy dissipation inequalities of Proposition 4.2 would fail, which undermines the subsequent convergence analysis.

- For all bounded set $E \subset \mathbf{R}^{d}$, set $\operatorname{diam}(E)=\sup _{x, \hat{x} \in E}|x-\hat{x}|$.

- We denote by $m_{E}$ the measure of an object $E$ in its natural dimension (i.e., the $d$-dimensional measure, if $E$ is a control volume, a dual control volume, a subdiamond or a diamond; and the $(d-1)$-dimensional measure, if $E$ is an interface or a part of an interface). According to Remark 3.1, for the definition of the scheme we could drop (19), in which case for a subdiamond $S_{K^{*}, L^{*}}^{K, L}$ such that $S_{K^{*}, L^{*}}^{K, L} \cap D^{K, L}=$ $\varnothing$ its volume is taken with the sign "minus".

3.2. Mesh parameters and regularity of meshes.

- We define the size of the mesh by $\operatorname{size}(\boldsymbol{T})=\max _{E \in \overline{\mathfrak{M}} \cup \overline{\mathfrak{M}^{*}} \cup \mathfrak{D}} \operatorname{diam}(E)$.

- Following [11, we call the maximum among

$$
\begin{gathered}
\max _{K^{*}} \operatorname{card}\left(\mathcal{N}^{*}\left(K^{*}\right)\right), \quad \max _{K} \frac{(\operatorname{diam}(K))^{d}}{m_{K}}, \max _{K^{*}} \frac{\left(\operatorname{diam}\left(K^{*}\right)\right)^{d}}{m_{K^{*}}}, \\
\max _{K \cap D \neq \varnothing}\left(\frac{\operatorname{diam}(K)}{\operatorname{diam}(D)}+\frac{\operatorname{diam}(D)}{\operatorname{diam}(K)}\right), \max _{K^{*} \cap D \neq \varnothing}\left(\frac{\operatorname{diam}\left(K^{*}\right)}{\operatorname{diam}(D)}+\frac{\operatorname{diam}(D)}{\operatorname{diam}\left(K^{*}\right)}\right),
\end{gathered}
$$

(where the maximums are taken over all $K \in \overline{\mathfrak{M}}, K^{*} \in \overline{\mathfrak{M}^{*}}, D \in \mathfrak{D}$ ) the regularity constant of the mesh and we denote it by $\operatorname{reg}(\boldsymbol{T})$. Roughly speaking, this constant controls the ratio of dimensions of neighbouring control volumes, diamonds and dual control volumes, as well as the proportions of each volume.

In all the discrete estimates and convergence results stated below, we require the family of meshes $\left(\boldsymbol{T}_{h}\right)_{h}$ to have regularity constants $\operatorname{reg}\left(\boldsymbol{T}_{h}\right)$ that are uniformly bounded in $h$. In the sequel, whenever there is a dependency of various constants on $\operatorname{reg}(\boldsymbol{T})$, we tacitly assume that this dependency is increasing.

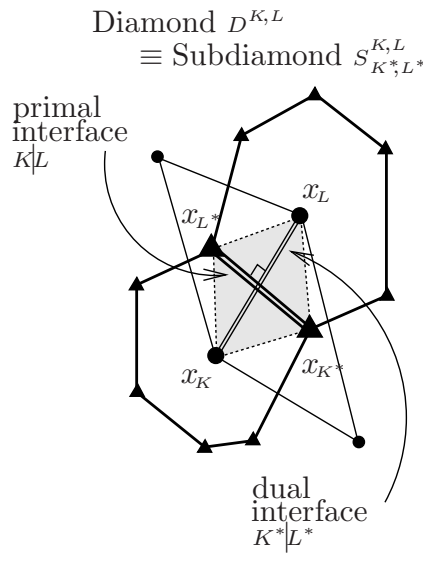

(a) 2D (sub)diamond.
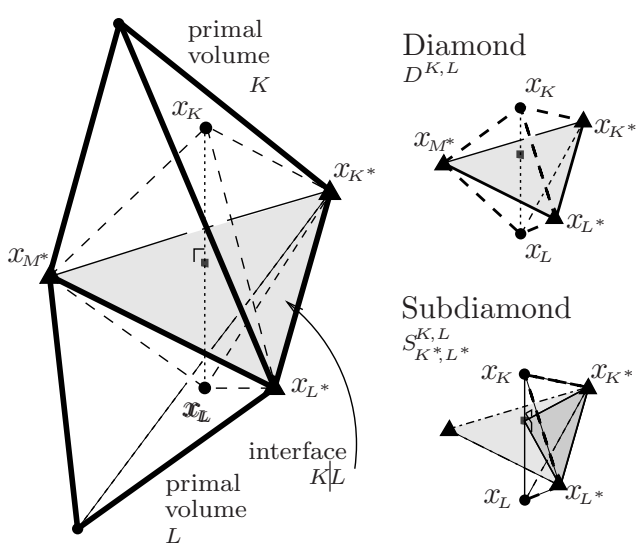

Subdiamond $S_{K^{*}, L^{*}}^{K, L}$

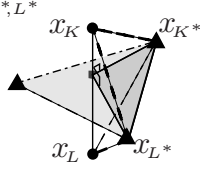

(b) 3D primal volumes, diamond, subdiamond.

Figure 2. Diamonds and subdiamonds. 
3.3. Discrete gradient and divergence operators. Diamonds permit to define the discrete gradient operator, while subdiamonds permit to define the discrete divergence operator (see (20), (21), (22) and (23), (24), (25) below, respectively). Both are needed to discretize the second order "diffusion" operator in equation (11). But first we need to introduce some more notation.

- (see Figure 31) For a subdiamond $S=S_{K^{*}, L^{*}}^{K, L}$, we denote by $\sigma=\sigma_{S}, \sigma^{*}=\sigma_{S}^{*}$ the (parts of the) interfaces $S \cap K \mid L$ and $S \cap K^{*} \mid L^{*}$, respectively, and by $\nu_{S}, \nu_{S}^{*}$, unit normal vectors to $\sigma_{S}$ and $\sigma_{S}^{*}$, respectively (their orientation is chosen arbitrarily).

- For a diamond $D=D^{K, L}$, we denote by $\operatorname{Proj}_{D}, \operatorname{Proj}_{D}^{*}$ the operators of orthogonal projection of $\mathbf{R}^{d}$ on the subspaces $\left\langle\overrightarrow{x_{K} x_{L}}>\right.$ and on $\left\langle\overrightarrow{x_{K} x_{L}}\right\rangle^{\perp}$, respectively. One should note that we have $\left\langle\nu_{S}\right\rangle=\left\langle\overrightarrow{x_{K} x_{L}}\right\rangle$ and $\left.\left\langle\nu_{S}^{*}\right\rangle \subset<\overrightarrow{x_{K} x_{L}}\right\rangle^{\perp}$ for all $S \in \mathfrak{S}$ such that $S \subset D$.
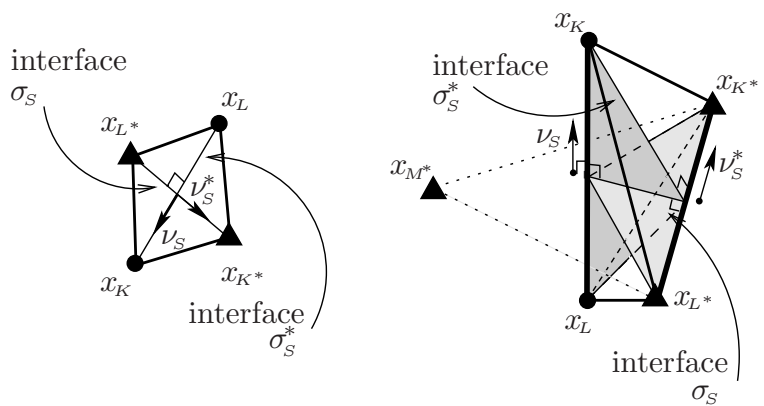

Figure 3. Notation in a subdiamond (2D and $3 \mathrm{D})$.

- For a couple of neighbours $K, L \in \overline{\mathfrak{M}}$, denote by $d_{K L}, d_{K, K L L}$, and $\nu_{K, L}$ the distance between $x_{K}$ and $x_{L}$, the distance from $x_{K}$ to $K \mid L$, and the unit normal vector to $K \mid L$ pointing from $K$ to $L$, respectively. More generally, if $K \in \mathfrak{M}$, then $\nu_{K}$ denotes the exterior unit normal vector to $\partial K$. In the same way, for neighbours $K^{*}, L^{*} \in \overline{\mathfrak{M}^{*}}$ we define $d_{K^{*} L^{*}}, d_{K^{*}, K^{*} \mid L^{*}}$, and $\nu_{K^{*}, L^{*}}$; for $K^{*} \in \overline{\mathfrak{M}^{*}}$, we define $\nu_{K^{*}}$.

Remark 3.2. Note that by construction both meshes $\overline{\mathfrak{M}}, \overline{\mathfrak{M}^{*}}$ are conformal (orthogonal) in the sense if [48); combined with the Delaunay condition, this means that $\nu_{K, L} \cdot \overrightarrow{x_{K} x_{L}}=d_{K L}, \nu_{K^{*}, L^{*}} \cdot \overrightarrow{x_{K^{*}} x_{L^{*}}}=d_{K^{*} L^{*}}$ for all neighbours $K, L$ and $K^{*}, L^{*}$, respectively.

The conformity property is particularly important for our $L^{1}$ framework imposed by the possible degeneracy of the diffusion term and the presence of the hyperbolic convective term. On the other hand, if this term is dropped, non-conformal double meshes can be considered for $d=2$ (see [57, 41, 11]) and $d=3$ (see [72, 39, 58, 8, [7, 38) within the variational framework.

- A discrete function on $\Omega$ is a set $w^{\mathfrak{T}}=\left(u^{\mathfrak{M}}, u^{\mathfrak{M} *}\right)$ consisting of two sets of real values $w^{\mathfrak{M}}=\left(w_{K}\right)_{K \in \mathfrak{M}}$ and $w^{\mathfrak{M}^{*}}=\left(w_{K^{*}}\right)_{K^{*} \in \mathfrak{M}^{*}}$. The set of all such functions is denoted by $\mathbf{R}^{\mathfrak{T}}$.

A discrete function on $\bar{\Omega}$ is a set

$$
w^{\overline{\mathfrak{T}}}=\left(w^{\mathfrak{M}}, w^{\mathfrak{M} *}, w^{\partial \mathfrak{M}}, w^{\partial \mathfrak{M}^{*}}\right) \equiv\left(w^{\mathfrak{T}}, w^{\partial \mathfrak{M}}, w^{\partial \mathfrak{M}^{*}}\right)
$$

consisting of four sets of real values

$$
w^{\mathfrak{M}}=\left(w_{K}\right)_{K \in \mathfrak{M}}, w^{\mathfrak{M} *}=\left(w_{K^{*}}\right)_{K^{*} \in \mathfrak{M}^{*}}, w^{\partial \mathfrak{M}}=\left(w_{K}\right)_{K \in \partial \mathfrak{M}}, w^{\partial \mathfrak{M}^{*}}=\left(w_{K^{*}}\right)_{K^{*} \in \partial \mathfrak{M}^{*}} .
$$


The set of all such functions is denoted by $\mathbf{R}^{\overline{\boldsymbol{\tau}}}$. In case all the components of $w^{\partial \mathfrak{M}}$ and of $w^{\partial \mathfrak{M}^{*}}$ are zero, we write $w^{\overline{\mathfrak{T}}} \in \mathbf{R}_{0}^{\overline{\mathfrak{I}}}$.

- A discrete field on $\Omega$ is a set $\mathcal{F}^{\mathfrak{T}}=\left(\mathcal{F}_{D}\right)_{D \in \mathfrak{D}}$ of vectors of $\mathbf{R}^{d}$. The set of all such functions is denoted by $\left(\mathbf{R}^{d}\right)^{\mathfrak{D}}$.

- On the set $\mathbf{R}^{\overline{\mathfrak{T}}}$ of discrete functions $w^{\overline{\mathfrak{T}}}$ on $\bar{\Omega}$, we define the discrete gradient operator $\nabla^{\mathfrak{T}}[\cdot]$ by

$$
\nabla^{\mathfrak{T}}: w^{\overline{\mathfrak{T}}} \in \mathbf{R}^{\overline{\mathfrak{T}}} \mapsto \nabla^{\mathfrak{T}} w^{\overline{\mathfrak{T}}}=\left(\nabla_{D} w^{\overline{\mathfrak{T}}}\right)_{D \in \mathfrak{D}} \in\left(\mathbf{R}^{d}\right)^{\mathcal{D}}
$$

where $\nabla^{\mathfrak{T}} w^{\overline{\mathfrak{T}}}$ is the discrete field on $\Omega$ with values

for $d=2$ :

$$
\nabla_{D} w^{\overline{\mathfrak{T}}}=\frac{w_{L}-w_{K}}{d_{K L}} \nu_{K, L}+\frac{w_{L^{*}}-w_{K^{*}}}{d_{K^{*} L^{*}}} \nu_{K^{*}, L^{*}} \quad \text { for } D=D^{K, L}=S_{K^{*}, L^{*}}^{K, L} ;
$$

for $d=3$ :

$$
\begin{aligned}
\nabla_{D} w^{\overline{\mathfrak{T}}}= & \frac{w_{L}-w_{K}}{d_{K L}} \nu_{K, L}+\frac{2}{m_{D}}\left(m_{S_{K^{*}, L^{*}}^{K, L}} \frac{w_{L^{*}}-w_{K^{*}}}{d_{K^{*} L^{*}}} \nu_{K^{*}, L^{*}}\right. \\
& \left.+m_{S_{L^{*}, M^{*}}^{K, L}} \frac{w_{M^{*}}-w_{L^{*}}}{d_{K^{*} L^{*}}} \nu_{L^{*}, M^{*}}+m_{S_{M^{*}, K^{*}}^{K, L}} \frac{w_{K^{*}}-w_{M^{*}}}{d_{M^{*} K^{*}}} \nu_{M^{*}, K^{*}}\right)
\end{aligned}
$$

Remark 3.3. Formulas (21) and (22) have the following common meaning. The vector $\nabla_{D} w^{\overline{\mathfrak{T}}}$ is the unique element of $\mathbf{R}^{d}$ such that $\operatorname{Proj}_{D}\left(\nabla_{D} w^{\overline{\mathfrak{T}}}\right)=\frac{w_{L^{*}}-w_{K^{*}}}{d_{K^{*} L^{*}}} \nu_{K^{*}, L^{*}}$. Further, for $d=2, \operatorname{Proj}_{D}^{*}\left(\nabla_{D} w^{\overline{\mathfrak{s}}}\right)$ is the gradient of the (unique) affine function on the interface $K \mid L$ (which is a segment with summits $x_{K^{*}}, x_{L^{*}}$ ) that takes the values $w_{K^{*}}, w_{L^{*}}$ at the points $x_{K^{*}}$ and $x_{L^{*}}$, respectively. Similarly, for $d=3$, $\operatorname{Proj}_{D}^{*}\left(\nabla_{D} w^{\overline{\mathfrak{s}}}\right)$ is the gradient of the (unique) affine function on the interface $K L$ (which is a triangle with summits $x_{K^{*}}, x_{L^{*}}, x_{M^{*}}$ ) that takes the values $w_{K^{*}}, w_{L^{*}}, w_{M^{*}}$ at the points $x_{K^{*}}, x_{L^{*}}, x_{M^{*}}$, respectively.

Thus, the primal mesh $\overline{\mathfrak{M}}$ serves to reconstruct one component of the gradient, which is the one in the direction $\overrightarrow{x_{K} x_{L}}$. The dual mesh $\overrightarrow{\mathfrak{M}^{*}}$ serves to reconstruct the $(d-1)$ other components which are the components in the $(d-1)$-dimensional hyperplane containing $K \mid L$ and is orthogonal to $\overrightarrow{x_{K} x_{L}}$.

The first and second assertions of Remark 3.3 are evident. Note that formula (21) easily generalizes to quite arbitrary non conformal double meshes (see [11, Lemma 2.4]). The third assertion is a direct consequence of the $2 \mathrm{D}$ reconstruction result of Lemma 9.6 given and proved in Appendix B (see also [8, 7]).

Remark 3.4. The discrete gradient is exact on affine functions. More precisely, let $D$ be a diamond $\left(D=D_{K^{*}, L^{*}}^{K, L}\right.$, if $d=2 ; D=D_{K^{*}, L^{*}, M^{*}}^{K, L}$, if $d=3$ ). Let $w(x):=w_{0}+r \cdot x$, $w_{0}, r \in \mathbf{R}^{d}$, be an affine function. If $w^{\overline{\mathfrak{T}}}$ is a discrete function with values

$$
\begin{aligned}
& w_{K}=w\left(x_{K}\right), w_{L}=w\left(x_{L}\right) ; \\
& w_{K^{*}}=w\left(x_{K^{*}}\right), w_{L^{*}}=w\left(x_{L^{*}}\right)\left(\text { and } w_{M^{*}}=w\left(x_{M^{*}}\right) \text { if } d=3\right),
\end{aligned}
$$

then $\nabla_{D} w^{\overline{\mathfrak{s}}}=r \equiv \nabla w$. This property follows by a straightforward comparison of the formulas (21) and (22) for the discrete gradient with the reconstruction formulas of the next lemma.

Lemma 3.1. Consider $D=D^{K, L} \in \mathfrak{D}$. With the notation above, for all $r \in \mathbf{R}^{d}$ one has the following reconstruction properties: 


$$
\begin{aligned}
& \text { for } d=2, \quad r=\left(r \cdot \nu_{K, L}\right) \nu_{K, L}+\left(r \cdot \nu_{K^{*}, L^{*}}\right) \nu_{K^{*}, L^{*}} \\
& \text { for } \begin{aligned}
=3, \\
r=\left(r \cdot \nu_{K, L}\right) \nu_{K, L}+\frac{2}{m_{D}}\left(m_{S_{K^{*}, L^{*}}^{K, L}}\left(r \cdot \nu_{K^{*}, L^{*}}\right) \nu_{K^{*}, L^{*}}+m_{S_{L^{*}, M^{*}}^{K, L}}\left(r \cdot \nu_{L^{*}, M^{*}}\right) \nu_{L^{*}, M^{*}}\right. \\
\\
\left.\quad+m_{S_{M^{*}, K^{*}}^{K, L}}\left(r \cdot \nu_{M^{*}, K^{*}}\right) \nu_{M^{*}, K^{*}}\right) .
\end{aligned}
\end{aligned}
$$

Proof. For $d=2$, the claim is a straightforward consequence of the conformity of the meshes (see Remark 13.2); $\nu_{K, L}, \nu_{K^{*} L^{*}}$ form an orthonormal basis of $\mathbf{R}^{2}$. When $d=3$, the claim follows from the orthogonality of $\nu_{K, L}$ to $K \mid L$ and from the 2D reconstruction property of Lemma 9.6 (cf. Appendix B) applied in the plane containing $K \mid L$.

Remark 3.5. The fourth assertion of Remark 3.3 indicates possible generalizations to the multi-dimensional case. Unfortunately, it can be shown that if $d \geq 4$, the direct generalization of the reconstitution formula of Lemma 3.1 holds only for meshes $\overline{\mathfrak{M}}$ with very special geometries, such as the uniform simplicial meshes (see Remark 9.2 which has to be combined with an induction argument on the dimension $d$ in order to link the weighted projections on the edges appearing in Lemma 3.1 with the weighted projections on the faces appearing in Lemma 9.6).

- For $S \in \mathfrak{S}$ such that $S \subset D$ with $D \in \mathfrak{D}$, we assign $\nabla_{S} u^{\mathfrak{T}}=\nabla_{D} u^{\mathfrak{T}}$. More generally, if $\mathcal{F}^{\mathfrak{T}}$ is a discrete field on $\Omega$, we assign $\mathcal{F}_{S}=\mathcal{F}_{D}$ for $S \subset D$.

For $K \in \mathfrak{M}$, we denote by $\mathcal{V}(K)$ the set of all subdiamonds $S \in \mathfrak{S}$ such that $K \cap S \neq \emptyset$. In the same way, for $K^{*} \in \overline{\mathfrak{M}^{*}}$ we define the set $\mathcal{\nu}^{*}\left(K^{*}\right)$ of subdiamonds intersecting $K^{*}$.

- On the set $\left(\mathbf{R}^{d}\right)^{\mathfrak{P}}$ of discrete fields $\mathcal{F}^{\mathfrak{T}}$, we define the discrete divergence operator $\operatorname{div}^{\mathfrak{T}}[\cdot]$ by

$$
\operatorname{div}^{\mathfrak{T}}: \mathcal{F}^{\mathfrak{T}} \in\left(\mathbf{R}^{d}\right)^{\mathfrak{P}} \mapsto v^{\mathfrak{T}}=\operatorname{div}^{\mathfrak{T}}\left[\mathcal{F}^{\mathfrak{T}}\right] \in \mathbf{R}^{\mathfrak{T}},
$$

where the discrete function $v^{\mathfrak{T}}=\left(v^{\mathfrak{M}}, v^{\mathfrak{M} *}\right)$ on $\Omega$ is given by

$$
\begin{aligned}
& v^{\mathfrak{M}}=\left(v_{K}\right)_{K \in \mathfrak{M}} \text { with } \quad v_{K}=\frac{1}{m_{K}} \sum_{S \in \mathcal{V}(K)} m_{\sigma_{S}} \mathcal{F}_{S} \cdot \nu_{K}, \text { where } \quad \nu_{K}=\left.\nu_{K}\right|_{S} ; \\
& v^{\mathfrak{M}^{*}}=\left(v_{K^{*}}\right)_{K^{*} \in \mathfrak{M}^{*}}, \quad v_{K^{*}}=\frac{1}{m_{K^{*}}} \sum_{S \in \mathcal{V}^{*}\left(K^{*}\right)} m_{\sigma_{S}^{*}} \mathcal{F}_{S} \cdot \nu_{K^{*}}, \quad \nu_{K^{*}}=\left.\nu_{K^{*}}\right|_{S} .
\end{aligned}
$$

In (24), (25) for $S$ given, $\nu_{K}=\left.\nu_{K}\right|_{S}$ denotes the restriction on $\sigma_{S}$ of the unit normal vector $\nu_{K}$ to $\partial K$ exterior to $K$; therefore it means the one of the vectors $\nu_{S},-\nu_{S}$ that is exterior to $K$ (see Figure 3). Similarly, $\nu_{K^{*}}=\left.\nu_{K^{*}}\right|_{S}$ is the one of the vectors $\nu_{S}^{*},-\nu_{S}^{*}$ that is exterior to $K^{*}$.

In fact, formulas (24), (25) can be conveniently expressed in terms of vector products involving the discrete field $\mathcal{F}_{S}$ and specific geometric objects depicted in Figure 3 (see [8, 7]).

3.4. Penalization operator. On the set $\mathbf{R}^{\overline{\underline{x}}}$ of discrete functions $w^{\overline{\underline{\Sigma}}}$ on $\bar{\Omega}$, we define the operator $\mathcal{P}^{\mathfrak{T}}[\cdot]$ of double mesh penalization by

$$
\mathcal{P}^{\mathfrak{T}}: w^{\overline{\mathfrak{T}}} \in \mathbf{R}^{\overline{\mathfrak{T}}} \mapsto v^{\mathfrak{T}}=\mathcal{P}^{\mathfrak{T}}\left[w^{\overline{\mathfrak{T}}}\right] \in \mathbf{R}^{\mathfrak{T}},
$$


where the discrete function $v^{\mathfrak{T}}=\left(v^{\mathfrak{M}}, v^{\mathfrak{M}^{*}}\right)$ on $\Omega$ is given by

$$
\begin{gathered}
v^{\mathfrak{M}}=\left(v_{K}\right)_{K \in \mathfrak{M}} \quad \text { with } \quad v_{K}=(d-1) \frac{1}{\operatorname{size}(\mathfrak{T})} \frac{1}{m_{K}} \sum_{K^{*} \in \overline{\mathfrak{M}^{*}}} m_{K \cap K^{*}}\left(w_{K}-w_{K^{*}}\right) \\
v^{\mathfrak{M} *}=\left(v_{K^{*}}\right)_{K^{*} \in \mathfrak{M}^{*}} \quad \text { with } \quad v_{K^{*}}=\frac{1}{\operatorname{size}(\mathfrak{T})} \frac{1}{m_{K^{*}}} \sum_{K \in \overline{\mathfrak{M}}} m_{K \cap K^{*}}\left(w_{K^{*}}-w_{K}\right) .
\end{gathered}
$$

The penalization is needed in order to ensure (without using the strong convergence of $\nabla^{\mathfrak{T}} w^{\overline{\mathfrak{T}}}$, cf. the proof of [11, Theorem 5.1]), that the two components of a discrete "double" function $w^{\overline{\mathfrak{s}}}$ converge to the same limit.

Remark 3.6. The choice of penalization operator we propose here is just the simplest possibility. In (26), (27), the difference $\left(w_{K^{*}}-w_{K}\right)$ could be replaced by $\left|w_{K^{*}}-w_{K}\right|^{p-2}\left(w_{K^{*}}-w_{K}\right)$, which seems more natural with respect to the assumptions on $\mathfrak{a}$; the power of $\operatorname{size}(\boldsymbol{T})$ in the denominator can be chosen arbitrarily. The convergence of the scheme would remain true. The question of optimal choice of the penalization operator is beyond the scope of this paper.

3.5. Discrete convection operator. Let $\mathfrak{f}: \mathbf{R} \rightarrow \mathbf{R}^{d}$ be continuous. Denote by $\omega_{M}(\cdot)$ a modulus of continuity of $\mathfrak{f}$ on $[-M, M]$, i.e., a continuous concave function on $[0, M]$ with $\omega_{M}(0)=0$ and

$$
\max _{a, b \in[-M, M],|a-b| \leq r}\|\mathfrak{f}(a)-\mathfrak{f}(b)\| \leq \omega_{M}(r) .
$$

Note that we can always choose $\omega_{M}$ strictly increasing, upon replacing $\omega_{M}$ by $\omega_{M}+I d$ if needed.

Following Eymard, Gallouët, Herbin 48, we now define discrete convection fluxes, separately for each of the meshes $\mathfrak{M}, \mathfrak{M}^{*}$. This will allow to discretize the convective part of equation (1).

- Let $K \mid L \in \mathcal{E}$. To approximate $f(u) \cdot \nu_{K, L}$ by means of the two values $u_{K}, u_{L}$ that are available in the neighbourhood of the interface $K \mid L$, let us use some function $g_{K, L}$ of the couple $\left(u_{K}, u_{L}\right) \in \mathbf{R}^{2}$. More exactly, take a collection of numerical convection flux functions $\left(g_{K, L}\right)_{K \mid L \in \mathcal{E}}, g_{K, L} \in C\left(\mathbf{R}^{2}, \mathbf{R}\right)$, with the following properties:

$$
\left\{\begin{array}{l}
\text { (a) } g_{K, L}(\cdot, b) \text { is nondecreasing for all } b \in \mathbf{R} \\
\text { and } g_{K, L}(a, \cdot) \text { is nonincreasing for all } a \in \mathbf{R} ; \\
\text { (b) } g_{K, L}(a, a)=\mathfrak{f}(a) \cdot \nu_{K, L} \text { for all } a \in \mathbf{R} \text {; } \\
\text { (c) } g_{K, L}(a, b)=-g_{K, L}(b, a) \forall a, b \in \mathbf{R} \text {, for all neighbours } K, L \in \overline{\mathfrak{M}} ; \\
\text { (d) } g_{K, L} \text { has the same modulus of continuity as } \mathfrak{f} \text {, i.e., } \\
\text { there exists } C \text { independent of } K \mid L \text { such that } \forall a, b, c, d \in[-M, M] \\
\quad\left|g_{K, L}(a, b)-g_{K, L}(c, d)\right| \leq C\left(\omega_{M}(|a-c|)+\omega_{M}(|b-d|)\right) .
\end{array}\right.
$$

These assumptions (see [48) are by now standard. Note that the assumption (28) (d) usually states that $\mathfrak{f}, g_{K, L}$ are Lipschitz continuous, with the same Lipschitz constant; here, we adapt it to the case of general continuous function $\mathfrak{f}$.

Note that (28) (b) and (c) are compatible. Also note that the consistency requirement (28) (b) together with the Green-Gauss formula imply

$$
\sum_{L \in \mathcal{N}(K)} m_{K \mid L} g_{K, L}(a, a)=\mathfrak{f}(a) \cdot \int_{\partial K} \nu_{K}=0 \quad \text { for all } a \in \mathbf{R}, \text { for all } K \in \mathfrak{M}
$$


Practical examples of numerical convective flux functions can be found in [48]. These include the Godunov, Lax-Friedrichs, Engquist-Osher and Rusanov fluxes as particular cases.

- Numerical convective flux functions $g_{K^{*}, L^{*}}, K^{*} L^{*} \in \mathcal{E}^{*}$, are defined similarly.

- On the set $\mathbf{R}^{\overline{\mathfrak{T}}}$ of discrete functions $u^{\overline{\mathfrak{T}}}$ on $\bar{\Omega}$, we define the operator $\left(\operatorname{div}_{c} \mathfrak{f}\right)^{\mathfrak{T}}[\cdot]$ of discrete convection by

$$
\left(\operatorname{div}_{c} \mathfrak{f}\right)^{\mathfrak{T}}: u^{\overline{\mathfrak{T}}} \in \mathbf{R}^{\overline{\mathfrak{T}}} \mapsto v^{\mathfrak{T}}=\left(\operatorname{div}_{c} \mathfrak{f}\right)^{\mathfrak{T}}\left[u^{\overline{\mathfrak{T}}}\right] \in \mathbf{R}^{\mathfrak{T}}
$$

where the discrete function $v^{\mathfrak{T}}=\left(v^{\mathfrak{M}}, v^{\mathfrak{M} *}\right)$ on $\Omega$ is given by

$$
\begin{gathered}
v^{\mathfrak{M}}=\left(v_{K}\right)_{K \in \mathfrak{M}} \quad \text { with } \quad v_{K}=\frac{1}{m_{K}} \sum_{L \in \mathcal{N}(K)} m_{K \mid L} g_{K, L}\left(u_{K}, u_{L}\right) ; \\
v^{\mathfrak{M}^{*}}=\left(v_{K^{*}}\right)_{K^{*} \in \mathfrak{M}^{*}}, \quad v_{K^{*}}=\frac{1}{m_{K^{*}}} \sum_{L^{*} \in \mathcal{N}^{*}\left(K^{*}\right)} m_{K^{*} L^{*}} g_{K^{*}, L^{*}}\left(u_{K^{*}}, u_{L^{*}}\right) .
\end{gathered}
$$

\subsection{Projection operators and test functions.}

- On $L^{1}(\Omega)$, we define the mesh projection operator $\mathbb{P}^{\mathfrak{T}}[\cdot]$ on the space of discrete functions on $\Omega$ by

$$
\mathbb{P}^{\mathfrak{T}}: \mathcal{S} \in L^{1}(\Omega) \mapsto \mathcal{S}^{\mathfrak{T}}=\mathbb{P}^{\mathfrak{T}}[\mathcal{S}] \in \mathbf{R}^{\mathfrak{T}},
$$

where the discrete function $\mathcal{S}^{\mathfrak{T}}=\left(\mathcal{S}^{\mathfrak{M}}, \mathcal{S}^{\mathfrak{M}^{*}}\right)$ on $\Omega$ is given by

$$
\begin{aligned}
\mathcal{S}^{\mathfrak{M}} & =\left(\mathcal{S}_{K}\right)_{K} \in \mathfrak{M} \text { with } \mathcal{S}_{K}=\frac{1}{m_{K}} \int_{K} \mathcal{S}(x) d x \\
\mathcal{S}^{\mathfrak{M}^{*}} & =\left(\mathcal{S}_{K^{*}}^{n}\right)_{K^{*} \in \mathfrak{M}^{*}} \text { with } \mathcal{S}_{K^{*}}=\frac{1}{m_{K^{*}}} \int_{K^{*}} \mathcal{S}(x) d x .
\end{aligned}
$$

- For a sufficiently regular function $\psi$ on $\bar{\Omega}$, we will often employ the notations $\psi^{\mathfrak{T}}=\mathbb{P}^{\mathfrak{T}}[\psi]$ and $(\nabla \psi)^{\mathfrak{T}}=\mathbb{P}^{\mathfrak{T}}[\nabla \psi]\left(\nabla \psi\right.$ being $\mathbf{R}^{d}$-valued, the projection is taken component per component). Further, for $K \mid L \in \mathcal{E}$ and $K^{*} L^{*} \in \mathcal{E}^{*}$, we introduce

$$
\psi_{K \mid L}=\frac{1}{m_{K \mid L}} \int_{K \mid L} \psi, \quad \psi_{K^{*} \mid L^{*}}=\frac{1}{m_{K^{*} \mid L^{*}}} \int_{K^{*} \mid L^{*}} \psi .
$$

For $L \in \partial \mathfrak{M}$, there exists $K L \subset \partial \Omega$ that coincides with $L$; in this case we assign $\psi_{L}=\psi_{K \mid L}$. If $\left.\psi\right|_{\partial \Omega}=0$, we have $\psi_{L}=0$ for all $L \in \partial \mathfrak{M}$. For $L^{*} \in \partial \mathfrak{M}^{*}$, we assign $\psi_{L^{*}}=\frac{1}{m_{L^{*}}} \int_{L^{*}} \psi$. If $\psi$ has a compact support in $\Omega$ and $\operatorname{size}(\mathfrak{T})$ is small enough, we have $\psi_{L^{*}}=0$ for all $L^{*} \in \partial \mathfrak{M}^{*}$.

Combining the above notation, we write $\psi^{\overline{\mathfrak{T}}}=\left(\mathbb{P}[\psi],\left(\psi_{K}\right)_{K \in \partial \mathfrak{M}},\left(\psi_{K^{*}}\right)_{K^{*} \in \partial \mathfrak{M}^{*}}\right)$ for the projection of a sufficiently regular function $\psi$ on the space $\mathbf{R}^{\overline{\mathfrak{T}}}$, and denote the corresponding projection operator by $\mathbb{P}^{\overline{\mathfrak{T}}}$.

\subsection{Dependency on $t$ and further notation.}

- Let $\boldsymbol{T}$ be a DDFV mesh as described above. Let $\Delta t>0$ be the time discretization step. Set $h=\max \{\operatorname{size}(\boldsymbol{T}), \Delta t\}$. By convention, we will use $h$ as the parameter for a sequence of finite volume schemes; our interest lies in studying convergence of corresponding discrete solutions as $h \downarrow 0$.

Denote by $N$ the integer part of $T / \Delta t$. In the sequel, in our notation we omit the dependency of $N, \boldsymbol{T}$ and $\Delta t$ on $h$.

- For a functional space $X$ on $\Omega$, we denote by $\mathbb{S}^{\Delta t}$ the projection operator

$$
\mathbb{S}^{\Delta t}: \mathcal{S} \in L^{1}(0, T ; X) \mapsto\left(\mathcal{S}^{n}\right)_{n=1, \ldots, N} \in(X)^{N}, \quad \mathcal{S}^{n}=\frac{1}{\Delta t} \int_{(n-1) \Delta t}^{n \Delta t} \mathcal{S}(t) d t
$$


- A discrete function on $Q$ is a set $u^{\mathfrak{T}, \Delta t}=\left(u^{\mathfrak{T}, n}\right)_{n=1, \ldots, N}$, where for each $n$, $u^{\mathfrak{T}, n}$ is a discrete function on $\Omega$. The set of all such functions is denoted $\mathbf{R}^{N \times \mathfrak{T}}$.

A discrete function on $\bar{Q}$ is a set $u^{\overline{\mathfrak{s}}, \overline{\Delta t}}=\left(u^{\overline{\mathfrak{\tau}}, n}\right)_{n=0, \ldots, N}$, where for each $n, u^{\overline{\mathfrak{\tau}}, n}$ is a discrete function on $\bar{\Omega}$. The set of all such functions is denoted by $\mathbf{R}^{(N+1) \times \overline{\boldsymbol{I}}}$. We also use discrete functions $u^{\overline{\mathfrak{T}}, \Delta t} \in \mathbf{R}^{N \times \overline{\mathfrak{T}}}$ and $u^{\mathfrak{T}, \overline{\Delta t}} \in \mathbf{R}^{(N+1) \times \mathfrak{T}}$. Each of $u^{\mathfrak{T}, \Delta t}, u^{\overline{\mathfrak{z}}, \Delta t}, u^{\mathfrak{\mathfrak { T }}, \overline{\Delta t}}$ is therefore a restriction of $u^{\overline{\mathfrak{z}}, \overline{\Delta t}}$. The entries of $u^{\overline{\mathfrak{z}}, n}$ are denoted by $u_{K}^{n}$ (respectively, $u_{K^{*}}^{n}$ ) for $K \in \mathfrak{M} \cup \partial \mathfrak{M}$ (respectively, for $K^{*} \in \mathfrak{M}^{*} \cup \partial \mathfrak{M}^{*}$ ).

A discrete field on $Q$ is a set $\mathcal{F}^{\mathfrak{T}, \Delta t}=\left(\mathcal{F}^{\mathfrak{T}, n}\right)_{n=1, \ldots, N}$ where for each $n, \mathcal{F}^{\mathfrak{T}, n}$ is a discrete field on $\Omega$. The set of all such fields is denoted by $\left(\mathbf{R}^{d}\right)^{N \times \mathfrak{D}}$.

- Any discrete function can be composed with a mapping $A: \mathbf{R} \rightarrow \mathbf{R}^{m}, m \in \mathbb{N}$; for instance, $A\left(u^{\overline{\mathfrak{s}}, \overline{\Delta t}}\right)$ stands for $w^{\overline{\mathfrak{s}}, \overline{\Delta t}}$ with values $w_{K}^{n}=A\left(u_{K}^{n}\right)$ for $K \in \overline{\mathfrak{M}}$ and $w_{K^{*}}^{n}=A\left(u_{K^{*}}^{n}\right)$ for $K^{*} \in \overline{\mathfrak{M}^{*}}$, for $n=0, \ldots, N$. Similarly, any discrete field can be composed with a mapping $\varphi: \mathbf{R}^{d} \rightarrow \mathbf{R}^{m}$; one has $\varphi\left(\mathcal{F}^{\mathfrak{T}}\right)=\left(\varphi\left(\mathcal{F}_{D}\right)\right)_{D \in \mathfrak{D}}$.

- We say that a discrete function is nonnegative ( respectively, nonpositive), if all its entries are nonnegative (respectively, nonpositive); e.g., for $v^{\mathfrak{T}} \in \mathbf{R}^{\mathfrak{T}}$ the notation $v^{\mathfrak{T}} \geq 0$ means that $v_{K} \geq 0$ for all $K \in \mathfrak{M}$ and $v_{K^{*}} \geq 0$ for all $K^{*} \in \mathfrak{M}^{*}$.

3.8. The finite volume scheme. With the notation introduced above, the finite volume discretization of problem (1) takes the following compact form:

find a discrete function $u^{\overline{\mathfrak{s}}, \overline{\Delta t}}$ on $\bar{Q}$ satisfying for $n=1, \ldots, N$ the equations

$\begin{aligned} \frac{u^{\mathfrak{T}, n}-u^{\mathfrak{T},(n-1)}}{\Delta t}+\left(\operatorname{div}_{c} \mathfrak{f}\right)^{\mathfrak{T}}\left[u^{\overline{\mathfrak{T}}, n}\right] & -\operatorname{div}^{\mathfrak{T}}\left[\mathfrak{a}\left(\nabla^{\mathfrak{T}} w^{\overline{\mathfrak{T}}, n}\right)\right] \\ & +\mathcal{P}^{\mathfrak{T}}\left[w^{\overline{\mathfrak{T}}, n}\right]=\mathbb{P}^{\mathfrak{T}}\left(\mathbb{S}^{\Delta t}[\mathcal{S}]\right)^{n}, \\ w^{\overline{\mathfrak{T}}}, n=A\left(u^{\overline{\mathfrak{T}}}, n\right), & \end{aligned}$

together with the boundary and initial conditions

$$
\text { for all } n=1, \ldots, N, \quad \begin{cases}u_{K}^{n}=0 & \text { for all } K \in \partial \mathfrak{M} \\ u_{K^{*}}^{n}=0 & \text { for all } K^{*} \in \partial \mathfrak{M}^{*}\end{cases}
$$

$$
\begin{cases}u_{K}^{0}=\frac{1}{m_{K}} \int_{K} u_{0} & \text { for all } K \in \mathfrak{M} \\ u_{K^{*}}^{0}=\frac{1}{m_{K^{*}}} \int_{K^{*}} u_{0} & \text { for all } K^{*} \in \mathfrak{M}^{*}\end{cases}
$$


Let us state (33) in a more explicit form:

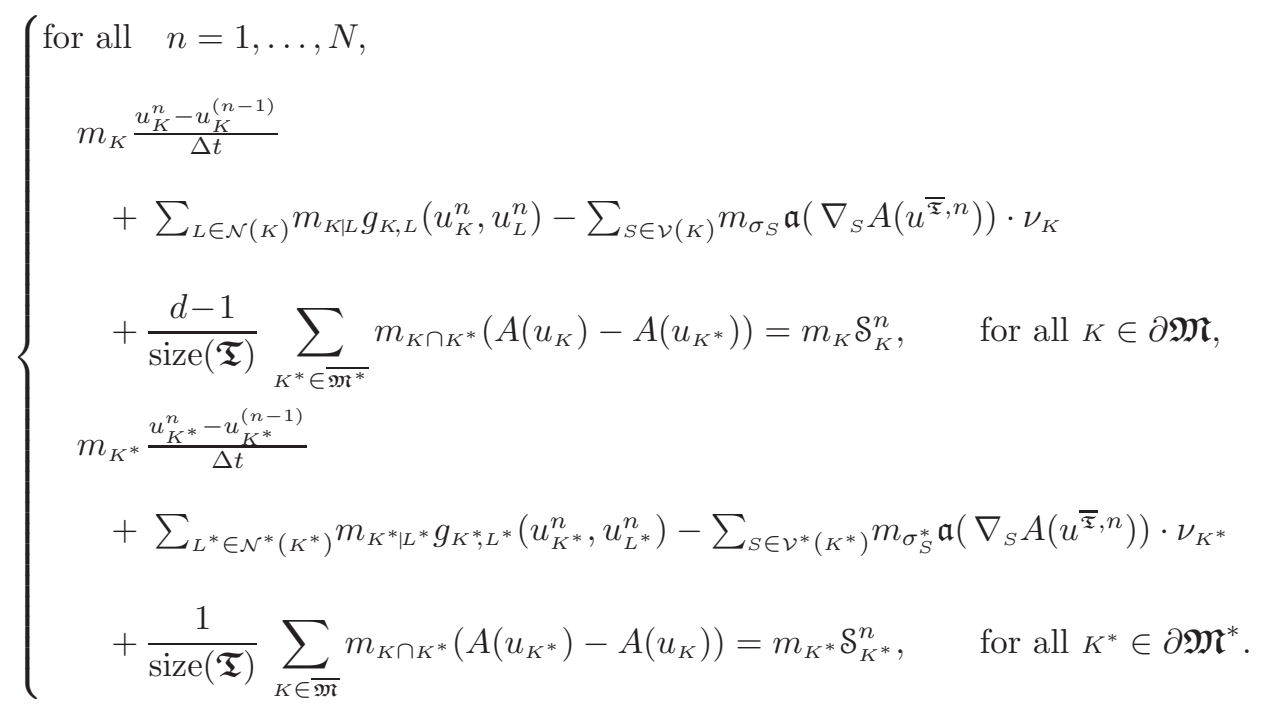

Here $\mathcal{S}_{K}^{n}, \mathcal{S}_{L}^{n}$ are given by (30), (32); $g_{K, L}, g_{K^{*}, L^{*}}$ are some numerical convection fuxes satisfying (28); $\nu_{K}, \nu_{K^{*}}$ for $S$ given have the same meaning as in (24), (25); finally, for $S$ given such that $S \subset D \in \mathfrak{D}, \nabla_{S} A\left(u^{\overline{\mathfrak{z}}}, n\right)$ is the vector of $\mathbf{R}^{d}$ constructed from the values $w_{K}=A\left(u_{K}^{n}\right), w_{K^{*}}=A\left(u_{K^{*}}^{n}\right)$ by formulas (21) (for $d=2$ ) or (22) (for $d=3)$, i.e., in the way indicated in Remark 3.3 .

\section{Elements of Discrete CAlCulus For DDFV SCHEMES}

In this section, we list convenient formulations of various summation-by-parts formulas and chain rules needed for the analysis of the discrete problem (33).

\subsection{Discrete duality formulas for the diffusion terms.}

- Recall that $\mathbf{R}^{\mathfrak{T}}$ is the space of all discrete functions on $\Omega$. For $m \in \mathbb{N}$ and $w^{\mathfrak{T}}, v^{\mathfrak{T}} \in\left(\mathbf{R}^{\mathfrak{T}}\right)^{m}$, set

$$
\llbracket w^{\mathfrak{T}}, v^{\mathfrak{T}} \rrbracket=\frac{1}{d} \sum_{K \in \mathfrak{M}} m_{K} w_{K} \cdot v_{K}+\frac{d-1}{d} \sum_{K^{*} \in \mathfrak{M}^{*}} m_{K^{*}} w_{K^{*}} \cdot v_{K^{*}}
$$

(here - denotes the scalar product in $\mathbf{R}^{m}$ ); it is clear that $\left.\llbracket \cdot, \cdot\right]$ is a scalar product on $\left(\mathbf{R}^{\mathfrak{T}}\right)^{m}$. We will use it for $m=1$ or $m=d$.

- Recall that $\left(\mathbf{R}^{d}\right)^{\mathcal{D}}$ is the space of all discrete fields on $\Omega$. For $\mathcal{F}^{\mathfrak{T}}, \mathcal{G}^{\mathfrak{T}} \in\left(\mathbf{R}^{d}\right)^{\mathfrak{D}}$, set

$$
\left\{\left\{\mathcal{F}^{\mathfrak{T}}, \mathcal{G}^{\mathfrak{T}}\right\}=\sum_{D \in \mathfrak{D}} m_{D} \mathcal{F}_{D} \cdot \mathcal{G}_{D}\right.
$$

it is clear that $\{\cdot, \cdot\}$ is a scalar product on $\left(\mathbf{R}^{d}\right)^{\mathcal{D}}$.

A key property of DDFV schemes (see [41, 11]) is the following discrete analogue of the duality between the $-\operatorname{div}[\cdot]$ and the $\nabla[\cdot]$ operators; it is sometimes called the discrete duality property for finite volumes. 
Proposition 4.1. Let $v^{\overline{\mathfrak{I}}} \in \mathbf{R}_{0}^{\overline{\mathfrak{I}}}$ and $\mathcal{F}^{\mathfrak{T}} \in\left(\mathbf{R}^{d}\right)^{\mathcal{D}}$. Then

$$
\llbracket-\operatorname{div}^{\mathfrak{T}}\left[\mathcal{F}^{\mathfrak{T}}\right], v^{\mathfrak{T}} \rrbracket=\left\{\left\{\mathcal{F}^{\mathfrak{T}}, \nabla^{\mathfrak{T}} v^{\overline{\mathfrak{T}}}\right\} .\right.
$$

Proof. The proof is straightforward, using the summation-by-parts procedure. Let us give it for the case $d=2$. Note that for $D=S=S_{K^{*}, L^{*}}^{K,{ }^{*}}, m_{S}=\frac{1}{2} m_{K \mid L} d_{K L}=$ $\frac{1}{2} m_{K^{*} L^{*}} d_{K^{*} L^{*}}$. By (36), by (24), (25), and finally by (21), (37), we get

$$
\begin{aligned}
& \llbracket-\operatorname{div}{ }^{\mathfrak{T}}\left[\mathcal{F}^{\mathfrak{T}}\right], v^{\mathfrak{s}} \rrbracket \\
& =-\frac{1}{2} \sum_{K \in \mathfrak{M}}\left(\sum_{S \in \mathcal{V}(K)} m_{\sigma_{S}} \mathcal{F}_{S} \cdot \nu_{K}\right) v_{K}-\frac{1}{2} \sum_{K^{*} \in \mathfrak{M}^{*}}\left(\sum_{S \in \mathcal{V}^{*}\left(K^{*}\right)} m_{\sigma_{S}^{*}} \mathcal{F}_{S} \cdot \nu_{K^{*}}\right) v_{K^{*}} \\
& =-\frac{1}{2} \sum_{K \in \overline{\mathfrak{M}}}\left(\sum_{S \in \mathcal{V}(K)} m_{\sigma_{S}} \mathcal{F}_{S} \cdot \nu_{K}\right) v_{K}-\frac{1}{2} \sum_{K^{*} \in \overline{\mathfrak{M}}}\left(\sum_{S \in \mathcal{V}^{*}\left(K^{*}\right)} m_{\sigma_{S}^{*}} \mathcal{F}_{S} \cdot \nu_{K^{*}}\right) v_{K^{*}} \\
& =\frac{1}{2} \sum_{S \in \mathfrak{S}, S=S_{K^{*}, L^{*}}^{K, L}} \mathcal{F}_{S} \cdot\left(m_{K \mid L}\left(v_{L}-v_{K}\right) \nu_{K, L}+m_{K^{*} \mid L^{*}}\left(v_{L^{*}}-v_{K^{*}}\right) \nu_{K^{*}, L^{*}}\right) \\
& =\sum_{S \in \mathfrak{G}, S=S_{K^{*}, L^{*}}^{K, L}} m_{S} \mathcal{F}_{S} \cdot\left(\frac{v_{L}-v_{K}}{d_{K L}} \nu_{K, L}+\frac{v_{L^{*}}-v_{K^{*}}}{d_{K^{*} L^{*}}} \nu_{K^{*}, L^{*}}\right) \\
& =\sum_{S \in \mathfrak{G}} m_{S} \mathcal{F}_{S} \cdot \nabla_{S} v^{\overline{\mathfrak{s}}}=\sum_{D \in \mathfrak{D}} m_{D} \mathcal{F}_{D} \cdot \nabla_{D} v^{\overline{\mathfrak{s}}}=\left\{\left\{\mathcal{F}^{\mathfrak{T}}, \nabla^{\mathfrak{T}} v^{\overline{\mathfrak{s}}}\right\} .\right.
\end{aligned}
$$

Furthermore, we have the following "entropy dissipation" inequalities:

Proposition 4.2. Let $u^{\overline{\boldsymbol{s}}} \in \mathbf{R}_{0}^{\overline{\mathbf{s}}}$ and $\psi \in \mathcal{D}(\bar{\Omega}), \psi \geq 0$. Let $\theta: \mathbf{R} \rightarrow \mathbf{R}$ be a nondecreasing function. Assume that

$$
\text { either } \theta(0)=0 \text {, or } \psi \in \mathcal{D}(\Omega) \text { and size }(\mathfrak{T}) \text { is small enough. }
$$

Denote $\psi^{\overline{\mathbf{s}}}=\mathbb{P}^{\overline{\mathfrak{x}}}[\psi]$. Then

$$
\begin{aligned}
\llbracket \operatorname{div}^{\mathfrak{T}}\left[k\left(\nabla^{\mathfrak{T}} A\left(u^{\overline{\mathfrak{T}}}\right)\right) \nabla^{\mathfrak{T}} A\left(u^{\overline{\mathfrak{T}}}\right)\right] & , \theta\left(u^{\mathfrak{T}}\right) \psi^{\mathfrak{T}} \rrbracket \\
& \leq-\left\{\left\{k\left(\nabla^{\mathfrak{T}} A\left(u^{\overline{\mathfrak{T}}}\right)\right) \nabla^{\mathfrak{T}} A_{\theta}\left(u^{\overline{\mathfrak{T}}}\right), \nabla^{\mathfrak{T}} \psi^{\overline{\mathfrak{T}}}\right\} .\right.
\end{aligned}
$$

Remark 4.1. Note that the conformity of the meshes (see Remark 3.2) is essential for this result, as well as the particular form of $\mathfrak{a}$ and (for $d=3$ ) condition (19).

Proof. Let us treat the left-hand side of (39) term by term. It is the sum of generic terms of the form $T_{K, S}, T_{K^{*}, S}^{*}$; here

$$
\begin{array}{r}
T_{K, S}=\frac{1}{d} m_{K} \frac{1}{m_{K}} m_{K \mid L} k\left(\nabla_{S} A\left(u^{\overline{\mathfrak{s}}}\right)\right) \nabla_{S} A\left(u^{\overline{\mathfrak{s}}}\right) \cdot \nu_{K, L} \theta\left(u_{K}\right) \psi_{K} \\
=\frac{1}{d} m_{K \mid L} k\left(\nabla_{S} A\left(u^{\overline{\mathfrak{s}}}\right)\right) \nabla_{S} A\left(u^{\overline{\mathfrak{\tau}}}\right) \cdot \nu_{K, L} \theta\left(u_{K}\right) \psi_{K}
\end{array}
$$

with $S=S^{K, L} \in \mathcal{V}(K)$. The notation $T_{K^{*}, S}^{*}$ stands for analogous terms involving $K^{*}$ and $S \in \mathcal{V}^{*}\left(K^{*}\right)$. Notice that thanks to assumption (38), $\theta\left(u^{\overline{\boldsymbol{s}}}\right) \psi^{\overline{\mathbf{s}}} \in \mathbf{R}_{0}^{\bar{\Sigma}}$, so that we can also add the terms $T_{K, S}, T_{K^{*}, S}^{*}$ corresponding to $K \in \partial \mathfrak{M}, K^{*} \in \partial \mathfrak{M}^{*}$, respectively. The summation of $T_{K, S}, T_{K^{*}, S}^{*}$ therefore runs on all subdiamonds $S=$ $S_{K^{*}, L^{*}}^{K, L} \in \mathfrak{S}$, with the associated $K, L \in \overline{\mathfrak{M}}, K^{*}, L^{*} \in \overline{\mathfrak{M}^{*}}$.

The convexity argument yields

$$
(A(z)-A(\hat{z})) \theta(\hat{z}) \leq A_{\theta}(z)-A_{\theta}(\hat{z}) \quad \text { for all } z, \hat{z} \in \mathbf{R} .
$$


By (20), using the positivity of $\psi_{K}$ and applying inequality (40), we get

$$
\begin{aligned}
T_{K, S}= & \frac{1}{d} m_{K \mid L} k\left(\nabla_{S} A\left(u^{\bar{\Sigma}}\right)\right) \frac{A\left(u_{L}\right)-A\left(u_{K}\right)}{d_{K L}} \theta\left(u_{K}\right) \psi_{K} \\
\leq & \frac{1}{d} m_{K \mid L} k\left(\nabla_{S} A\left(u^{\bar{\Sigma}}\right)\right) \frac{A_{\theta}\left(u_{L}\right)-A_{\theta}\left(u_{K}\right)}{d_{K L}} \psi_{K} .
\end{aligned}
$$

The terms $T_{K^{*}, S}$ are treated in the same way. Now by the same computation as in the proof of Proposition 4.1, one shows that the right-hand sides of (41) and of the corresponding inequality for $T_{K^{*}, S}$ sum up to yield the right-hand side of (39). This concludes the proof.

4.2. Summation formulas for the penalization terms. For the penalization operator $\mathcal{P}^{\mathfrak{z}}$, we have the following summation formulas.

Lemma 4.1. Let $w^{\overline{\mathfrak{x}}} \in \mathbf{R}^{\overline{\mathfrak{x}}}$ and $\psi^{\overline{\mathfrak{x}}} \in \mathbf{R}_{0}^{\overline{\mathfrak{s}}}$. Then

$$
\llbracket \mathcal{P}\left[w^{\overline{\mathfrak{s}}}\right], \psi^{\mathfrak{s}} \rrbracket=\frac{d-1}{d} \sum_{K \in \overline{\mathfrak{M}}, K^{*} \in \overline{\mathfrak{M}^{*}}} m_{K \cap K^{*}} \frac{\left(w_{K}-w_{K^{*}}\right)\left(\psi_{K}-\psi_{K^{*}}\right)}{\operatorname{size}(\mathfrak{T})} .
$$

Further, let $A, \theta: \mathbf{R} \rightarrow \mathbf{R}$ be nondecreasing. Assume $u^{\overline{\underline{\Sigma}}} \in \mathbf{R}^{\overline{\underline{\Sigma}}}$ is such that $A\left(u^{\overline{\mathbf{s}}}\right)$ belongs to $\mathbf{R}_{0}^{\bar{\Sigma}}$. Let $\psi \in \mathcal{D}(\bar{\Omega}), \psi \geq 0$; denote $\psi^{\overline{\mathfrak{s}}}=\mathbb{P}^{\overline{\mathfrak{s}}}[\psi]$. Assume (38). Then

$$
\llbracket \mathcal{P}\left[A\left(u^{\overline{\mathfrak{T}}}\right)\right], \theta\left(u^{\mathfrak{T}}\right) \psi^{\mathfrak{T}} \rrbracket \geq \frac{d-1}{d} \sum_{K \in \overline{\mathfrak{M}}, K^{*} \in \overline{\mathfrak{M}^{*}}} m_{K \cap K^{*}} \theta\left(u_{K}\right) \frac{\left(A\left(u_{K}\right)-A\left(u_{K^{*}}\right)\right)\left(\psi_{K}-\psi_{K^{*}}\right)}{\operatorname{size}(\mathfrak{T})} .
$$

In both formulas (42), (43), the values $\psi_{K}, \psi_{K^{*}}$ for $K \in \overline{\mathfrak{M}}, K^{*} \in \overline{\mathfrak{M}^{*}}$ are those of the corresponding discrete function $\psi^{\overline{\mathrm{s}}}$.

The proof is straightforward from the definitions of $\llbracket \cdot, \cdot \rrbracket$ and $\mathcal{P}^{\mathfrak{z}}$, using the summation-by-parts procedure.

4.3. Discrete duality formulas for the evolution terms.

Lemma 4.2. Let $\theta: \mathbf{R} \rightarrow \mathbf{R}$ be a nondecreasing function, and $\eta=\int \theta(s) d s$ be its primitive. Let $\psi \in \mathcal{D}(\bar{Q}), \psi \geq 0$. Denote $\psi^{\mathfrak{T}, \Delta t}=\mathbb{P}^{\mathfrak{T}} \circ \mathbb{S}^{\Delta t}[\psi]$. Then for all $u^{\mathfrak{s}, \overline{\Delta t}} \in \mathbf{R}^{(N+1) \times \mathfrak{z}}$ one has

$$
\begin{aligned}
\sum_{n=1}^{N} \Delta t \llbracket \frac{u^{\mathfrak{T}, n}-u^{\mathfrak{T},(n-1)}}{\Delta t}, \theta\left(u^{\mathfrak{T}, n}\right) \psi^{\mathfrak{T}, n} \rrbracket \\
\geq-\sum_{n=1}^{N-1} \Delta t \llbracket \eta\left(u^{\mathfrak{T}, n}\right), \frac{\psi^{\mathfrak{T},(n+1)}-\psi^{\mathfrak{T}, n}}{\Delta t} \rrbracket \\
\quad+\llbracket \eta\left(u^{\mathfrak{T}, N}\right), \psi^{\mathfrak{T}, N} \rrbracket-\llbracket \eta\left(u^{\mathfrak{T}, 0}\right), \psi^{\mathfrak{T}, 1} \rrbracket .
\end{aligned}
$$

Proof. The formula follows by the Abel transformation combined with the convexity inequality: $(z-\hat{z}) \theta(z) \geq \eta(z)-\eta(\hat{z}) \quad$ for all $z, \hat{z} \in \mathbf{R}$.

4.4. Discrete duality formulas for the convection terms. For the convection terms, we have a more involved "entropy dissipation" duality formula. For later use, we state it in the double framework, although each of the meshes $\mathfrak{M}, \mathfrak{M}^{*}$ is treated separately in the proof. 
Proposition 4.3. Let $u^{\bar{\Sigma}} \in \mathbf{R}_{0}^{\bar{\Sigma}}$ and $\psi \in \mathcal{D}(\bar{\Omega})$. Let $\theta: \mathbf{R} \rightarrow \mathbf{R}$ be a nondecreasing function. Assume (38). Consider the associated entropy-flux pair

$$
\eta=\int \theta(s) d s, \quad \mathfrak{q}=\theta \mathfrak{f}-\int \mathfrak{f}(s) d \theta(s) .
$$

Denote $\psi^{\mathfrak{T}}=\mathbb{P}^{\mathfrak{T}}[\psi]$ and $(\nabla \psi)^{\mathfrak{T}}=\mathbb{P}^{\mathfrak{T}}[\nabla \psi]$. One has

$$
\begin{aligned}
& \llbracket\left(\operatorname{div}_{c} \mathfrak{f}\right)^{\mathfrak{T}}\left[u^{\overline{\mathfrak{x}}}\right], \theta\left(u^{\mathfrak{T}}\right) \psi^{\mathfrak{T}} \rrbracket \\
& =-\llbracket \mathfrak{q}\left(u^{\mathfrak{T}}\right),(\nabla \psi)^{\mathfrak{T}} \rrbracket+I_{\theta}\left[u^{\mathfrak{M}}, \psi\right]+R_{\theta}\left[u^{\mathfrak{M}}, \psi\right]+I_{\theta}^{*}\left[u^{\mathfrak{M} \mathfrak{N}^{*}}, \psi\right]+R_{\theta}^{*}\left[u^{\mathfrak{M}^{*}}, \psi\right],
\end{aligned}
$$

where

$$
I_{\theta}\left[u^{\mathfrak{M}}, \psi\right]=\frac{1}{d} \sum_{K \mid L \in \mathcal{E}} m_{K \mid L} I_{\theta}^{K \mid L} \psi_{K \mid L}, I_{\theta}^{*}\left[u^{\mathfrak{M} *}, \psi\right]=\frac{d-1}{d} \sum_{K^{*} \mid L^{*} \in \mathcal{E}^{*}} m_{K^{*} \mid L^{*}} I_{\theta}^{K^{*} \mid L^{*}} \psi_{K^{*} \mid L^{*}}
$$

with

$$
\begin{aligned}
& I_{\theta}^{K \mid L}=\int_{u_{K}}^{u_{L}}\left(g_{K, L}(s, s)-g_{K, L}\left(u_{K}, u_{L}\right)\right) d \theta(s), \\
& I_{\theta}^{K^{*} L^{*}}=\int_{u_{K^{*}}}^{u_{L^{*}}}\left(g_{K^{*}, L^{*}}(s, s)-g_{K^{*}, L^{*}}\left(u_{K^{*}}, u_{L^{*}}\right)\right) d \theta(s) .
\end{aligned}
$$

Further, one has $I_{\theta}^{K \mid L} \geq 0$ for all $\kappa \mid L \in \mathcal{E}$, and the remainder term $R_{\theta}$ satisfies

$$
\begin{aligned}
& \left|R_{\theta}\left[u^{\mathfrak{M}}, \psi\right]\right| \leq\left(\max _{K \in \mathfrak{M}}\left|\theta\left(u_{K}\right)\right|\right) \sum_{K \mid L} m_{K \mid \mathcal{E}}\left(R_{K}^{K \mid L}+R_{L}^{K \mid L}\right)\left(\left|\psi_{K}-\psi_{K \mid L}\right|+\left|\psi_{L}-\psi_{K \mid L}\right|\right), \\
& R_{K}^{K \mid L}=\left|g_{K, L}\left(u_{K}, u_{K}\right)-g_{K, L}\left(u_{K}, u_{L}\right)\right|, \quad R_{L}^{K \mid L}=\left|g_{K, L}\left(u_{L}, u_{L}\right)-g_{K, L}\left(u_{K}, u_{L}\right)\right| .
\end{aligned}
$$

Similarly, one has $I_{\theta}^{K^{*} L^{*}} \geq 0$ for all $K^{*} \mid L^{*} \in \mathcal{E}^{*}$, and the remainder term $R_{\theta}^{*}$ satisfies the analogue of (477), (48) with $K, L, \mathfrak{M}, \mathcal{E}$ replaced by $K^{*}, L^{*}, \mathfrak{M}^{*}, \mathcal{E}^{*}$.

Note that our notation is consistent: we have $I_{\theta}^{K \mid L}=I_{\theta}^{L \mid K}, R_{K}^{K \mid L}=R_{L}^{L \mid K}$ for all neighbours $K, L$ (for dual neighbours $K^{*}, L^{*}$, similar identities hold).

Proof. We exploit the ideas of [48] and [29].

Thanks to (38) and because $u^{\bar{\tau}}$ is zero on boundary volumes, we have

$$
\eta\left(u_{L}\right) \psi_{L}=0 \text { for all } L \in \partial \mathfrak{M} ; \quad \eta\left(u_{L^{*}}\right) \psi_{L^{*}}=0 \text { for all } L^{*} \in \partial \mathfrak{M}^{*} .
$$

Separating the contributions of $\mathfrak{M}$ and $\mathfrak{M}^{*}$, we write the left-hand side of (44) as $\frac{1}{d} I+\frac{d-1}{d} I^{*}$, where

$$
I:=\sum_{K \in \mathfrak{M}} m_{K}\left(\frac{1}{m_{K}} \sum_{L \in \mathcal{N}(K)} m_{K \mid L} g_{K, L}\left(u_{K}, u_{L}\right)\right) \theta\left(u_{K}\right) \psi_{K} .
$$

Applying (28) (c) and (29), using (49) in the summation-by-parts procedure, we get

$$
\begin{aligned}
I=\sum_{K \mid L \in \mathcal{E}} m_{K \mid L}\left(\theta\left(u_{L}\right)\left(g_{K, L}\left(u_{L}, u_{L}\right)-g_{K, L}\left(u_{K}, u_{L}\right)\right) \psi_{L}\right. & \\
& \left.-\theta\left(u_{K}\right)\left(g_{K, L}\left(u_{K}, u_{K}\right)-g_{K, L}\left(u_{K}, u_{L}\right)\right) \psi_{K}\right) .
\end{aligned}
$$


Hence, choosing $\psi_{K \mid L}$ as defined in (31), we have

$$
\begin{gathered}
I=\sum_{K \mid L \in \mathcal{E}} m_{K \mid L}\left(\theta\left(u_{L}\right)\left(g_{K, L}\left(u_{L}, u_{L}\right)-g_{K, L}\left(u_{K}, u_{L}\right)\right)\right. \\
\left.-\theta\left(u_{K}\right)\left(g_{K, L}\left(u_{K}, u_{K}\right)-g_{K, L}\left(u_{K}, u_{L}\right)\right)\right) \psi_{K \mid L} \\
+\sum_{K \mid L \in \mathcal{E}} m_{K \mid L}\left(\theta\left(u_{L}\right)\left(g_{K, L}\left(u_{L}, u_{L}\right)-g_{K, L}\left(u_{K}, u_{L}\right)\right)\left(\psi_{L}-\psi_{K \mid L}\right)\right. \\
\left.-\theta\left(u_{K}\right)\left(g_{K, L}\left(u_{K}, u_{K}\right)-g_{K, L}\left(u_{K}, u_{L}\right)\right)\left(\psi_{K}-\psi_{K \mid L}\right)\right) .
\end{gathered}
$$

Now recall that $g=g_{K, L}$ satisfies (28)(b). Thus the following integration-by-parts formula holds true:

$$
\begin{aligned}
& (\mathfrak{q}(b)-\mathfrak{q}(a)) \cdot \nu_{K, L}=\left(\theta(b) \mathfrak{f}(b)-\theta(a) \mathfrak{f}(a)-\int_{a}^{b} \mathfrak{f}(s) d \theta(s)\right) \cdot \nu_{K, L} \\
& =\theta(b)(g(b, b)-g(a, b))-\theta(a)(g(a, a)-g(a, b))-\int_{a}^{b}(g(s, s)-g(a, b)) d \theta(s) .
\end{aligned}
$$

We deduce $I=J+I_{\theta}+R_{\theta}$, where

$$
\begin{aligned}
J & =\sum_{K \mid L \in \mathcal{E}} m_{K \mid L}\left(\mathfrak{q}\left(u_{K}\right)-\mathfrak{q}\left(u_{L}\right)\right) \cdot \nu_{K, L} \psi_{K \mid L}=\sum_{K \in \mathfrak{M}} \mathfrak{q}\left(u_{K}\right) \cdot\left(\sum_{L \in \mathcal{N}(K)} m_{K \mid L} \psi_{K \mid L} \nu_{K, L}\right) \\
& =\sum_{K \in \mathfrak{M}} \mathfrak{q}\left(u_{K}\right) \cdot \int_{\partial K} \psi \nu_{K}=\sum_{K \in \mathfrak{M}} \int_{K} \operatorname{div}\left(\mathfrak{q}\left(u_{K}\right) \psi\right)=\sum_{K \in \mathfrak{M}} \int_{K} \mathfrak{q}\left(u_{K}\right) \cdot \nabla \psi \\
& =\sum_{K \in \mathfrak{M}} m_{K} \mathfrak{q}\left(u_{K}\right) \cdot(\nabla \psi)_{K}
\end{aligned}
$$

and

$$
\begin{gathered}
I_{\theta}=\sum_{K \mid L \in \mathcal{E}} m_{K \mid L}\left(\int_{u_{K}}^{u_{L}}\left(g_{K, L}(s, s)-g_{K, L}\left(u_{K}, u_{L}\right)\right) d \theta(s)\right) \psi_{K \mid L} \\
\left|R_{\theta}\right| \leq \sum_{K \mid L \in \mathcal{E}} m_{K \mid L}\left(\left|g_{K, L}\left(u_{K}, u_{K}\right)-g_{K, L}\left(u_{K}, u_{L}\right)\right|+\left|g_{K, L}\left(u_{L}, u_{L}\right)-g_{K, L}\left(u_{K}, u_{L}\right)\right|\right) \\
\times\left(\left|\psi_{K}-\psi_{K \mid L}\right|+\left|\psi_{L}-\psi_{K \mid L}\right|\right) \times\left(\max _{K \in \mathfrak{M}}\left|\theta\left(u_{K}\right)\right|\right) .
\end{gathered}
$$

In the same way, $I^{*}=J^{*}+I_{\theta}^{*}+R_{\theta}^{*}$ with analogous estimates. We have the equality $\frac{1}{d} J+\frac{d-1}{d} J^{*}=\llbracket \mathfrak{q}\left(u^{\mathfrak{T}}\right),(\nabla \psi)^{\mathfrak{T}} \rrbracket$. With the notation of (45)-(48) the result of the proposition follows.

\section{Properties of Discrete OPERATORS AND FUnCtIONAL SPACES}

In this section we state important embedding and compactness properties of spaces of discrete functions, as well as the asymptotic (as $h \rightarrow 0$ ) properties of various discrete operators. 


\subsection{Discrete functions and fields as elements of Lebesgue spaces.}

For any $E \subset \bar{Q}$, denote by $\mathbb{1}_{E}$ its characteristic function.

For $n=1, \ldots, N$, set

$$
\begin{aligned}
Q_{K}^{n} & =[(n-1) \Delta t, n \Delta t[\times K, & \text { for } K \in \mathfrak{M} ; \\
Q_{K^{*}}^{n} & =\left[(n-1) \Delta t, n \Delta t\left[\times K^{*},\right.\right. & \text { for } K^{*} \in \mathfrak{M}^{*} ; \\
Q_{D}^{n} & =[(n-1) \Delta t, n \Delta t[\times D, & \text { for } D \in \mathfrak{D} .
\end{aligned}
$$

For a discrete function $v^{\mathfrak{s}, \Delta t}$ on $Q$, denote by $v^{\mathfrak{M}, \Delta t}$ (respectively, by $v^{\mathfrak{M}{ }^{*}, \Delta t}$ ) the piecewise constant function

$$
\begin{aligned}
& v^{\mathfrak{M}, \Delta t}(t, x)=\sum_{n=1}^{N} \sum_{K \in \mathfrak{M}} u_{K}^{n} 1_{Q_{K}^{n}}(t, x) \\
& \quad\left(\text { respectively, } v^{\mathfrak{M}^{*}, \Delta t}(t, x)=\sum_{n=1}^{N} \sum_{K^{*} \in \mathfrak{M}^{*}} u_{K^{*}}^{n} \mathbb{1}_{Q_{K^{*}}^{n}}(t, x)\right) .
\end{aligned}
$$

Whenever it is convenient, we identify the discrete function $v^{\mathfrak{x}, \Delta t} \in \mathbf{R}^{N \times \mathfrak{x}}$ with the function on $Q$ given by

$$
v^{\mathfrak{s}, \Delta t}(t, x)=\frac{1}{d} v^{\mathfrak{M}, \Delta t}(t, x)+\frac{d-1}{d} v^{\mathfrak{M}^{*}, \Delta t}(t, x) .
$$

In a similar way, we identify a discrete field $\mathcal{F}^{\mathfrak{T}, \Delta t} \in \mathbf{R}^{N \times \mathfrak{P}}$ on $Q$ with the function

$$
\mathcal{F}^{\mathfrak{T}, \Delta t}(t, x)=\sum_{n=1}^{N} \sum_{D \in \mathcal{O}} \mathcal{F}_{D}^{n} \mathbb{1}_{Q_{D}^{n}}(t, x) .
$$

Analogous conventions apply to time-independent discrete functions and discrete fields, in which case we suppress the superscript $\Delta t$ in the notation.

5.2. Consistency properties of discrete operators. In the proposition below we show the consistency properties of the projection and discrete gradient operators in Lebesgue spaces. Also note the property (iv), which, combined with formula (42), expresses the fact that the penalization operator introduced in Section 3.4 vanishes (in an appropriate sense) as $\operatorname{size}(\mathfrak{T}) \rightarrow 0$.

Proposition 5.1. Let $\mathfrak{T}$ be a double mesh of $\Omega, \Delta t>0, h=\max \{\operatorname{size}(\mathfrak{T}), \Delta t\}$, and $q \in[1,+\infty]$. Then

(i) there exists a constant $C$ that only depends on $\Omega, q$ and $\operatorname{reg}(\mathfrak{T})$ such that

$$
\forall w \in L^{q}(Q),\left\|\left(\mathbb{P}^{\mathfrak{T}} \circ \mathbb{S}^{\Delta t} w\right)^{\mathfrak{M}, \Delta t}\right\|_{L^{q}}+\left\|\left(\mathbb{P}^{\mathfrak{T}} \circ \mathbb{S}^{\Delta t} w\right)^{\mathfrak{M}^{*}, \Delta t}\right\|_{L^{q}} \leq C\|w\|_{L^{q}},
$$

and

$$
\left.\forall w \in L^{q}\left(0, T ; W_{0}^{1, q}(\Omega)\right)\right),\left\|\nabla^{\mathfrak{z}} \mathbb{P}^{\overline{\mathfrak{s}}} \circ \mathbb{S}^{\Delta t} w\right\|_{L^{q}} \leq C\|\nabla w\|_{L^{q}}
$$

(ii) for all $w \in L^{q}(Q), q<+\infty$, both $\left(\mathbb{P}^{\mathfrak{I}} \circ \mathbb{S}^{\Delta t} w\right)^{\mathfrak{M}, \Delta t}$ and $\left(\mathbb{P}^{\mathfrak{I}} \circ \mathbb{S}^{\Delta t} w\right)^{\mathfrak{M} *}, \Delta t$ converge to $w$ in $L^{q}(Q)$ as $h \rightarrow 0$;

(iii) for all $w \in L^{q}\left(0, T ; W_{0}^{1, q}(\Omega)\right), q<+\infty$, the discrete fields $\nabla^{\mathfrak{T}} \mathbb{P}^{\overline{\mathfrak{z}}} \circ \mathbb{S}^{\Delta t} w$ converge to $\nabla w$ in $\left(L^{q}(Q)\right)^{d}$ as $h \rightarrow 0$; 
(iv) let $\psi \in \mathcal{D}(\bar{\Omega})$, and $\psi^{\overline{\mathbf{s}}, n}=\mathbb{P}^{\overline{\mathfrak{s}}}(\mathbb{S}[\psi])^{n}, n=1, \ldots, N$. There exists a constant $C$ that only depends on $Q$ and $\operatorname{reg}(\mathfrak{T})$ such that

$$
\sum_{n=1}^{N} \Delta t \sum_{K \in \overline{\mathfrak{M}}, K^{*} \in \overline{\mathfrak{M}^{*}}} m_{K \cap K^{*}} \frac{\left(\psi_{K}-\psi_{K^{*}}\right)^{2}}{\operatorname{size}(\mathfrak{T})} \leq C\|\nabla \psi\|_{L^{\infty}} \times \operatorname{size}(\mathfrak{T}) .
$$

Proof. The proof of (i)-(iii) is a straightforward generalization of [1], Lemma 3.3, Proposition 3.4 and Corollary 3.5]. We need to take into account the fact that $\left\|\mathbb{S}^{\Delta t} w\right\|_{L^{q}(X)} \leq\|w\|_{L^{q}(X)}$ and (for $\left.q \neq+\infty\right)\left\|\mathbb{S}^{\Delta t} w-w\right\|_{L^{q}(X)} \rightarrow 0$ as $\Delta t \rightarrow 0$ for all $w \in L^{q}(0, T ; X)$, where $X$ stands for $L^{q}(\Omega)$ or for $W_{0}^{1, q}(\Omega)$. Remark 3.4 is important for (iii) (thus, the Delaunay property of $\mathfrak{M}$ is used). Further, in a standard way similar to [11, Lemma 3.3] one proves that for all $K \in \overline{\mathfrak{M}}, K^{*} \in \overline{\mathfrak{M}^{*}}$ such that $K \cap K^{*} \neq \varnothing$, one has $\left|\psi_{K}^{n}-\psi_{K^{*}}^{n}\right| \leq C(\operatorname{reg}(\mathfrak{T}))\|\nabla \psi\|_{L^{\infty}} \times \operatorname{size}(\mathfrak{T})$ for all $n=1, \ldots, N$. Hence the claim (iv) follows.

5.3. Discrete embedding and compactness results. Next we state a version of the Poincaré inequality and an embedding-kind translation estimate on double discrete functions.

Proposition 5.2. Assume $\mathfrak{T}$ is a double mesh on $\Omega, \Delta t>0$. Let $q \in[1,+\infty)$. There exists a constant $C>0$ that only depends on $\operatorname{diam}(\Omega)$ and $q$ such that

(i) for all $w^{\overline{\mathfrak{s}}, \Delta t} \in \mathbf{R}_{0}^{N \times \overline{\mathfrak{s}}}$ one has $\left\|w^{\mathfrak{M}, \Delta t}\right\|_{L^{q}}+\left\|w^{\mathfrak{M}^{*}, \Delta t}\right\|_{L^{q}} \leq C\left\|\nabla^{\mathfrak{\mathfrak { I }}} w^{\overline{\mathfrak{s}}, \Delta t}\right\|_{q}$;

(ii) for all $w^{\overline{\mathbf{s}}} \in \mathbf{R}_{0}^{\overline{\mathbf{\Sigma}}}$, for all $\Delta \in \mathbf{R}^{d}$ one has

$$
\left\|w^{\mathfrak{M}}(\cdot+\Delta)-w^{\mathfrak{M}}(\cdot)\right\|_{L^{q}}+\left\|w^{\mathfrak{M}^{*}}(\cdot+\Delta)-w^{\mathfrak{M}^{*}}(\cdot)\right\|_{L^{q}} \leq C\left\|\nabla^{\mathfrak{T}} w^{\overline{\mathfrak{x}}}\right\|_{L^{q}} \times|\Delta|^{1 / q} .
$$

Proof. The proof follows the lines of [12, Lemma 1] and [11, Lemma 3.6]. Note that if $d=3$, the fact that all interfaces $K \mid L$ are triangles plays an important role in the proof.

Here is the asymptotic compactness result for "discrete $L^{p}\left(0, T ; W_{0}^{1, p}(\Omega)\right.$ " spaces.

Proposition 5.3. Let $p \in(1,+\infty)$. Assume we are given a family $\left\{w^{\bar{\Sigma}}, \Delta t\right\}_{h}$ of discrete functions in $\mathbf{R}_{0}^{N \times \overline{\boldsymbol{x}}}$ corresponding to a family of double meshes $\mathfrak{T}$ such that $\operatorname{reg}(\mathfrak{T})$ is uniformly bounded (recall that we parametrize the meshes by $h=$ $\max \{\operatorname{size}(\mathfrak{T}), \Delta t\})$.

(i) Assume that there exists a constant $C>0$ such that

$$
\left\|\nabla^{\mathfrak{\tau}} w^{\overline{\mathfrak{s}}, \Delta t}\right\|_{L^{p}} \leq C \text {. }
$$

Then there exists a (not labelled) sequence of meshes such that as $h \rightarrow 0$

$$
w^{\mathfrak{\tau}, \Delta t}=\frac{1}{d} w^{\mathfrak{m}, \Delta t}+\frac{d-1}{d} w^{\mathfrak{M}^{*}, \Delta t} \text { converge weakly in } L^{p}(Q) \text { to some limit } w ;
$$

furthermore, $w \in L^{p}\left(0, T ; W_{0}^{1, p}(\Omega)\right)$ and

the discrete fields $\nabla^{\mathfrak{T}} w^{\overline{\mathfrak{x}}, \Delta t}$ converge weakly in $\left(L^{p}(Q)\right)^{d}$ to $\nabla w$ as $h \rightarrow 0$.

(ii) If, in addition,

$$
\sum_{n=1}^{N} \Delta t \llbracket \mathcal{P}^{\mathfrak{T}}\left[w^{\overline{\mathfrak{s}}, n}\right], w^{\mathfrak{s}, n} \rrbracket \leq C,
$$

where $\mathcal{P}^{\mathfrak{T}}$ are the penalization operators introduced in Section 3.4, then

$$
\text { both } w^{\mathfrak{M}, \Delta t} \text { and } w^{\mathfrak{M}^{*}, \Delta t} \text { converge to } w \text { weakly in } L^{p}(Q) \text { as } h \rightarrow 0 \text {. }
$$


Remark 5.1. Note that upon providing uniform estimates on time translates of $w^{\mathfrak{\tau}, \Delta t}$ in $L^{p}(Q)$, strong convergence to $w$ in $L^{p}(Q)$ holds true (see Section 7 ).

Proof. (i) The proof is very similar to the one of [11, Lemma 3.8].

First, by Proposition $5.2(\mathrm{i})$, both families $\left\{w^{\mathfrak{m}, \Delta t}\right\}_{h},\left\{w^{\mathfrak{M} *}, \Delta t\right\}_{h}$ of components of $w^{\mathfrak{T}, \Delta t}$ are bounded in $L^{p}(Q)$. Therefore we can choose a common sequence such that both components converge weakly in $L^{p}(Q)$. Also $w^{\mathfrak{T}, \Delta t}=\frac{1}{d} w^{\mathfrak{m}, \Delta t}+\frac{d-1}{d} w^{\mathfrak{M}{ }^{*}, \Delta t}$ converge weakly to some limit that we denote $w$. We can also assume that the corresponding sequence $\left\{\nabla^{\mathfrak{T}} w^{\overline{\mathfrak{s}}, \Delta t}\right\}_{h}$ converges weakly in $\left(L^{p}(Q)\right)^{d}$ to some limit $\chi$. Let us show that $w \in L^{p}\left(0, T ; W_{0}^{1, p}(\Omega)\right)$ and $\chi=\nabla w$.

Take any field $\mathcal{F} \in\left(L^{p^{\prime}}\left(0, T ; W^{1, p^{\prime}}(\Omega)\right)^{d}\right.$. Denote by $\mathcal{F}^{\mathfrak{x}, \Delta t}$ the discrete field on $Q$ with entries

$$
\mathcal{F}_{D}^{n}=\frac{1}{\Delta t \times m_{D}} \int_{(n-1) \Delta t}^{n \Delta t} \int_{D} \mathcal{F}
$$

Denote by $(\operatorname{div} \mathcal{F})^{\mathfrak{T}, \Delta t}$ the discrete function $\mathbb{P}^{\mathfrak{T}} \circ \mathbb{S}^{\Delta t}[\operatorname{div} \mathcal{F}]$ on $Q$, which has the entries

$$
\begin{gathered}
(\operatorname{div} \mathcal{F})_{K}^{n}=\frac{1}{\Delta t m_{K}} \int_{(n-1) \Delta t}^{n \Delta t} \int_{K} \operatorname{div} \mathcal{F}=\frac{1}{\Delta t m_{K}} \int_{(n-1) \Delta t}^{n \Delta t} \sum_{S \in \mathcal{V}(K)} \int_{\sigma_{S}} \mathcal{F} \cdot \nu_{K}, \\
(\operatorname{div} \mathcal{F})_{K^{*}}^{n}=\frac{1}{\Delta t m_{K^{*}}} \int_{(n-1) \Delta t}^{n \Delta t} \int_{K^{*}} \operatorname{div} \mathcal{F}=\frac{1}{\Delta t m_{K^{*}}} \int_{(n-1) \Delta t}^{n \Delta t} \sum_{S \in \mathcal{V}^{*}\left(K^{*}\right)} \int_{\sigma_{S}^{*}} \mathcal{F} \cdot \nu_{K^{*}} .
\end{gathered}
$$

By Proposition 4.1 by definitions of $\{[, \cdot\}\}, \llbracket \cdot, \cdot]$ and using the notation introduced in Section 5.1 we have

$$
\begin{aligned}
& 0=\sum_{n=1}^{N} \Delta t\left\{\left\{\mathcal{F}^{\mathfrak{T}, n}, \nabla^{\mathfrak{T}} w^{\overline{\mathfrak{T}}, n}\right\}+\sum_{n=1}^{N} \Delta t \llbracket \operatorname{div}^{\mathfrak{T}}\left[\mathcal{F}^{\mathfrak{T}, n}\right], w^{\mathfrak{T}, n} \rrbracket\right. \\
& =\sum_{n=1}^{N} \Delta t\left\{\left\{\mathcal{F}^{\mathfrak{s}, n}, \quad \nabla^{\mathfrak{T}} w^{\overline{\mathfrak{s}}, n}\right\}+\sum_{n=1}^{N} \Delta t \llbracket(\operatorname{div} \mathcal{F})^{\mathfrak{s}, n}, w^{\mathfrak{s}, n} \rrbracket\right. \\
& +\sum_{n=1}^{N} \Delta t \llbracket \operatorname{div}^{\mathfrak{T}}\left[\mathcal{F}^{\mathfrak{T}, n}\right]-(\operatorname{div} \mathcal{F})^{\mathfrak{T}, n}, w^{\mathfrak{T}, n} \rrbracket \\
& =\int_{Q} \mathcal{F}^{\mathfrak{T}, \Delta t} \cdot \nabla^{\mathfrak{T}} w^{\overline{\mathfrak{I}}, \Delta t}+\int_{Q}(\operatorname{div} \mathcal{F})\left(\frac{1}{d} w^{\mathfrak{M}, \Delta t}+\frac{d-1}{d} w^{\mathfrak{M} *, \Delta t}\right) \\
& +\sum_{n=1}^{N} \Delta t \llbracket \operatorname{div}^{\mathfrak{T}}\left[\mathcal{F}^{\mathfrak{T}, n}\right]-(\operatorname{div} \mathcal{F})^{\mathfrak{T}, n}, w^{\mathfrak{T}, n} \rrbracket .
\end{aligned}
$$

As in Proposition [5.1] one shows that $\left\|\mathcal{F}^{\mathfrak{z}, n}-\mathcal{F}\right\|_{L^{p^{\prime}}}$ tends to zero as $h \rightarrow 0$. Therefore we deduce

$$
0=\int_{Q} \mathcal{F} \cdot \chi+\int_{Q}(\operatorname{div} \mathcal{F}) w+\lim _{h \rightarrow 0} \sum_{n=1}^{N} \Delta t \llbracket \operatorname{div}^{\mathfrak{T}}\left[\mathcal{F}^{\mathfrak{T}, n}\right]-(\operatorname{div} \mathcal{F})^{\mathfrak{s}, n}, w^{\mathfrak{s}, n} \rrbracket .
$$


By definition of $\llbracket \cdot, \cdot \rrbracket$, we have

$$
\begin{aligned}
& \left.\sum_{n=1}^{N} \Delta t \llbracket \operatorname{div}^{\mathfrak{T}}\left[\mathcal{F}^{\mathfrak{T}, n}\right]-(\operatorname{div} \mathcal{F})^{\mathfrak{T}, n}, w^{\mathfrak{T}, n}\right] \\
& =\frac{1}{d} \sum_{n=1}^{N} \Delta t\left(m_{K} w_{K}^{n} \frac{1}{\Delta t m_{K}} \int_{(n-1) \Delta t}^{n \Delta t} \sum_{S \in \mathcal{V}(K)}\left(\int_{\sigma_{S}} \mathcal{F}-m_{\sigma_{S}} \mathcal{F}_{S}^{n}\right) \cdot \nu_{K}\right) \\
& +\frac{d-1}{d} \sum_{n=1}^{N} \Delta t\left(m_{K^{*}} w_{K^{*}}^{n} \frac{1}{\Delta t m_{K^{*}}} \int_{(n-1) \Delta t}^{n \Delta t} \sum_{S \in \mathcal{V}^{*}\left(K^{*}\right)}\left(\int_{\sigma_{S}^{*}} \mathcal{F}-m_{\sigma_{S}^{*}} \mathcal{F}_{S}^{n}\right) \cdot \nu_{K^{*}}\right) .
\end{aligned}
$$

Denote by $R+R^{*}$ the right-hand side above. Summing by parts, we get

$$
R=\frac{1}{d} \sum_{n=1}^{N} \sum_{K \mid L \in \mathcal{E}} d_{K L} \int_{(n-1) \Delta t}^{n \Delta t} \int_{K \mid L}\left(\mathcal{F}-\frac{1}{m_{D}} \int_{D} \mathcal{F}\right) \cdot \nu_{K, L} \frac{w_{K}^{n}-w_{L}^{n}}{d_{K L}}
$$

where $D$ stands for the diamond $D^{K L}$ containing the interface $K \mid L$. By the Hölder inequality, we deduce that $|R|$ is controlled by

$$
\begin{gathered}
\left(\sum_{n=1}^{N} \sum_{K \mid L \in \mathcal{E}} d_{K L} \int_{(n-1) \Delta t}^{n \Delta t} \int_{K \mid L}\left|\mathcal{F}-\frac{1}{m_{D}} \int_{D} \mathcal{F}\right|^{p^{\prime}}\right)^{\frac{1}{p^{\prime}}} \\
\times\left(\sum_{n=1}^{N} \Delta t \sum_{K \mid L \in \mathcal{E}} m_{K \mid L} d_{K L}\left|\frac{w_{K}^{n}-w_{L}^{n}}{d_{K L}}\right|^{p}\right)^{\frac{1}{p}} .
\end{gathered}
$$

Using standard estimates similar to [11, Lemma 3.2] and the definition of $\nabla^{\mathfrak{T}} w^{\overline{\mathfrak{T}}, \Delta t}$, we conclude that

$$
\begin{gathered}
|R| \leq C(\operatorname{reg}(\mathfrak{T})) \times \operatorname{size}(\mathfrak{T}) \times\|\mathcal{F}\|_{L^{p^{\prime}}\left(W^{1, p^{\prime}}\right)}\left\|\nabla^{\mathfrak{T}} w^{\overline{\mathfrak{T}}, \Delta t}\right\|_{L^{p}} \\
\leq C(\operatorname{reg}(\mathfrak{T})) \times h \times\|\mathcal{F}\|_{L^{p^{\prime}}\left(W^{1, p^{\prime}}\right)} \times C \rightarrow 0
\end{gathered}
$$

as $h \rightarrow 0$. In the same way, we find $\left|R^{*}\right| \rightarrow 0$ as $h \rightarrow 0$.

Thus for all $\mathcal{F} \in\left(L^{p^{\prime}}\left(0, T ; W^{1, p^{\prime}}(\Omega)\right)^{d}\right.$, the last term in (51) is zero, so that $w \in L^{p}\left(0, T ; W_{0}^{1, p}(\Omega)\right)$ and $\chi=\nabla w$.

(ii) If also $\sum_{n=1}^{N} \Delta t \llbracket \mathcal{P}^{\mathfrak{T}}\left[w^{\overline{\mathfrak{T}}, n}\right], w^{\mathfrak{T}, n} \rrbracket \leq C$, then by Lemma 4.1 with $\psi^{\overline{\mathfrak{T}}}=w^{\overline{\mathfrak{T}}}$ we get

$$
\frac{d-1}{d} \sum_{n=1}^{N} \Delta t \sum_{K \in \overline{\mathfrak{M}}, K^{*} \in \overline{\mathfrak{M}^{*}}} m_{K \cap K^{*}}\left(w_{K}^{n}-w_{K^{*}}^{n}\right)^{2} \leq C h .
$$

This means that $\left\|w^{\mathfrak{M}, \Delta t}-w^{\mathfrak{M}^{*}, \Delta t}\right\|_{L^{2}} \rightarrow 0$ as $h \rightarrow 0$, which permits to identify the weak limits of both $w^{\mathfrak{M}, \Delta t}$ and $w^{\mathfrak{M}^{*}, \Delta t}$ with $w$.

\section{Properties of Discrete solutions}

\subsection{A priori estimates.}

Proposition 6.1. Assume we are given a family of double meshes $\boldsymbol{T}$ of $\Omega$ and associated time steps $\Delta t$ such that $h=\max \{\operatorname{size}(\boldsymbol{T}), \Delta t\} \rightarrow 0$. Assume that $\operatorname{reg}(\mathfrak{T})$ is uniformly bounded.

Let $u^{\overline{\mathfrak{s}}, \overline{\Delta t}}$ be a solution to (33), (34), (35) (recall that $w^{\overline{\mathfrak{s}}, \overline{\Delta t}}=A\left(u^{\overline{\mathfrak{s}}, \overline{\Delta t}}\right)$ ). Then the following a priori estimates hold uniformly in $h$ : 
(i) $\max \left\{\left\|u^{\mathfrak{m}, \Delta t}\right\|_{L^{\infty}},\left\|u^{\mathfrak{M} \mathfrak{m}^{*}, \Delta t}\right\|_{L^{\infty}}\right\} \leq M:=\left\|u_{0}\right\|_{L^{\infty}}+\int_{0}^{T}\|\mathcal{S}(t, \cdot)\|_{L^{\infty}} d t$;

(ii) there exists $C>0$ such that

$$
\left\|\nabla^{\mathfrak{\tau}} w^{\overline{\mathfrak{s}}, \Delta t}\right\|_{L^{p}} \leq C \quad \text { and } \quad \sum_{n=1}^{N} \Delta t \llbracket \mathcal{P}^{\mathfrak{\tau}}\left[w^{\overline{\mathfrak{s}}, n}\right], w^{\mathfrak{T}, n} \rrbracket \leq C ;
$$

(iii) there exists $C>0$ such that (with the notation of Proposition 4.3)

$$
\sum_{n=1}^{N} \Delta t\left(I_{\mathrm{Id}}\left[u^{\mathfrak{m}, n}, 1\right]+I_{\mathrm{Id}}^{*}\left[u^{\mathfrak{M} *, n}, 1\right]\right) \leq C ;
$$

(iv) there exists a modulus of continuity $\omega_{A}(\cdot)$ such that for all $\Delta>0$,

$$
\int_{Q}\left|w^{\mathfrak{M}, \Delta t}(t+\Delta, x)-w^{\mathfrak{M}, \Delta t}(t, x)\right|+\left|w^{\mathfrak{M}^{*}, \Delta t}(t+\Delta, x)-w^{\mathfrak{M}^{*}, \Delta t}(t, x)\right| \leq \omega_{A}(\Delta),
$$

where $w^{\mathfrak{M}, \Delta t}, w^{\mathfrak{M}^{*}, \Delta t}$ are extended by zero on $(N \Delta t,+\infty) \times \Omega$.

Proof. (i) Denote $\mathcal{S}^{i}=\left(\mathbb{S}^{\Delta t}[\mathcal{S}]\right)^{i}$ and $\mathcal{S}^{\mathfrak{T}, i}=\mathbb{P}^{\mathfrak{T}}\left[\left(\mathbb{S}^{\Delta t}[\mathcal{S}]\right)^{i}\right]$. For $n=0, \ldots, N$, set $c^{n}=\left\|u_{0}\right\|_{L^{\infty}}+\sum_{i=1}^{n} \Delta t\left\|\mathcal{S}^{i}\right\|_{L^{\infty}}$; note that $c^{n} \leq\left\|u_{0}\right\|_{L^{\infty}}+\int_{0}^{T}\|\mathcal{S}(t, \cdot)\|_{L^{\infty}} d t=M$ for all $n=1, \ldots, N$.

Let us prove by induction that $\left\|u^{\mathfrak{m}, n}\right\|_{L^{\infty}} \leq c^{n},\left\|u^{\mathfrak{M}^{*}, n}\right\|_{L^{\infty}} \leq c^{n}$. This claim is clear for $n=0$. Assume it holds true for $n=k-1$. Take the scalar product $\llbracket \cdot, \cdot]$ of equations (33) corresponding to $n=k$ with the discrete function $\theta\left(u^{\mathfrak{\tau}, k}\right):=$ $\operatorname{sign}^{+}\left(u^{\mathfrak{T}, k}-c^{k}\right)$. We get

$$
\begin{aligned}
& \llbracket \frac{u^{\mathfrak{T}, k}-u^{\mathfrak{T},(k-1)}}{\Delta t}-\mathcal{S}^{\mathfrak{T}, k}, \theta\left(u^{\mathfrak{T}, k}\right) \rrbracket+\llbracket\left(\operatorname{div}_{c} \mathfrak{f}\right)^{\mathfrak{T}}\left[u^{\overline{\mathfrak{T}}, k}\right], \theta\left(u^{\mathfrak{\mathfrak { T }}, k}\right) \rrbracket \\
& -\llbracket \operatorname{div}^{\mathfrak{\mathfrak { T }}}\left[\mathfrak{a}\left(\nabla^{\mathfrak{T}} A\left(u^{\overline{\mathfrak{T}}, k}\right)\right)\right], \theta\left(u^{\mathfrak{T}, k}\right) \rrbracket+\llbracket \mathcal{P}^{\mathfrak{T}}\left[w^{\overline{\mathfrak{T}}, k}\right], \theta\left(u^{\mathfrak{T}, k}\right) \rrbracket=0 .
\end{aligned}
$$

Let us apply to the last three terms above Proposition 4.3. Proposition 4.2 and Lemma 4.1 respectively, with $\psi \equiv 1$. Note that $\theta(0)=0$, so that (38) holds. We conclude that each of the three last terms in (52) is nonnegative. Hence

$$
\begin{aligned}
0 & \geq \llbracket \frac{u^{\mathfrak{T}, k}-u_{K}^{\mathfrak{T},(k-1)}}{\Delta t}-\mathcal{S}^{\mathfrak{T}, k}, \theta\left(u^{\mathfrak{T}, k}\right) \rrbracket \\
& =\llbracket \frac{\left(u^{\mathfrak{T}, k}-c^{k}\right)-\left(u^{\mathfrak{T},(k-1)}-c^{(k-1)}\right)}{\Delta t}+\left(\left\|\mathcal{S}^{k}\right\|_{L^{\infty}}-\mathcal{S}^{\mathfrak{T}, k}\right), \operatorname{sign}^{+}\left(u^{\mathfrak{T}, k}-c^{k}\right) \rrbracket \\
& \geq \llbracket \frac{\left(u^{\mathfrak{T}, k}-c^{k}\right)-\left(u^{\mathfrak{T},(k-1)}-c^{(k-1)}\right)}{\Delta t}, \operatorname{sign}^{+}\left(u^{\mathfrak{T}, k}-c^{k}\right) \rrbracket \\
& \geq \llbracket\left(u^{\mathfrak{T}, k}-c^{k}\right)^{+}-\left(u^{\mathfrak{T},(k-1)}-c^{(k-1)}\right)^{+}, 1^{\mathfrak{T}} \rrbracket,
\end{aligned}
$$

where $1^{\mathfrak{z}}=\mathbb{P}^{\mathfrak{x}}[1]$. By the induction hypothesis we deduce that $\left(u^{\mathfrak{T}, k}-c^{k}\right)^{+} \leq 0$, which proves our claim for $n=k$. 
(ii) For $n=1, \ldots, N$, take the scalar product $\llbracket \cdot, \cdot \rrbracket$ of equations (33) with the discrete function $w^{\mathfrak{\tau}, n}=A\left(u^{\mathfrak{\tau}, n}\right)$. Multiply by $\Delta t$ and sum up in $n$. We get

$$
\begin{aligned}
& \sum_{n=1}^{N} \Delta t \llbracket \frac{u^{\mathfrak{s}, n}-u^{\mathfrak{T},(n-1)}}{\Delta t}, A\left(u^{\mathfrak{T}, n}\right) \rrbracket+\sum_{n=1}^{N} \Delta t \llbracket\left(\operatorname{div}_{c} \mathfrak{f}\right)^{\mathfrak{T}}\left[u^{\overline{\mathfrak{T}}, n}\right], A\left(u^{\mathfrak{T}, n}\right) \rrbracket \\
& -\sum_{n=1}^{N} \Delta t \llbracket \operatorname{div}^{\mathfrak{T}}\left[\mathfrak{a}\left(\nabla^{\mathfrak{T}} w^{\overline{\mathfrak{T}}, n}\right)\right], w^{\mathfrak{T}, n} \rrbracket+\sum_{n=1}^{N} \Delta t \llbracket \mathcal{P}^{\mathfrak{T}}\left[w^{\overline{\mathfrak{s}}, n}\right], w^{\mathfrak{T}, n} \rrbracket \\
& =\sum_{n=1}^{N} \Delta t \llbracket \mathbb{P}^{\mathfrak{T}}\left(\mathbb{S}^{\Delta t}[\mathcal{S}]\right)^{n}, w^{\mathfrak{T}, n} \rrbracket .
\end{aligned}
$$

Note that with $\theta(\cdot)=A(\cdot)$ and $\psi \equiv 1$, (38) holds. Applying Lemma 4.2, Proposition 4.3. Proposition 4.1 and Lemma 4.1, respectively, to the terms on the left-hand side of (53), we find

$$
\begin{aligned}
& \llbracket \eta\left(u^{\mathfrak{T}, N}\right), 1^{\mathfrak{T}} \rrbracket+\sum_{n=1}^{N} \Delta t\left(I_{A}\left[u^{\mathfrak{M}, n}, 1\right]+I_{A}^{*}\left[u^{\mathfrak{M} *}, n, 1\right]\right) \\
& \quad+\sum_{n=1}^{N} \Delta t\left\{\left\{\mathfrak{a}\left(\nabla^{\mathfrak{T}} w^{\overline{\mathfrak{s}}, n}\right), \nabla^{\mathfrak{T}} w^{\overline{\mathfrak{s}}, n}\right\}+\sum_{n=1}^{N} \Delta t \llbracket \mathcal{P}^{\mathfrak{T}}\left[w^{\overline{\mathfrak{s}}, n}\right], w^{\mathfrak{s}, n} \rrbracket\right. \\
& =\sum_{n=1}^{N} \Delta t \llbracket \mathbb{P}^{\mathfrak{T}}\left(\mathbb{S}^{\Delta t}[\mathcal{S}]\right)^{n}, w^{\mathfrak{T}, n} \rrbracket+\llbracket B_{A}\left(u^{\mathfrak{T}, 0}\right), 1^{\mathfrak{T}} \rrbracket,
\end{aligned}
$$

where $B_{A}(z)=\int_{0}^{z} A(s) d s$ and $I_{A}, I_{A}^{*}$ are defined in Proposition 4.3 The first two terms in (54) are nonnegative; the next one is lower bounded by a constant times $\left(\left\|\nabla^{\mathfrak{T}} w^{\overline{\mathfrak{s}}}, \Delta t\right\|_{L^{p}}\right)^{p}$ due to the coercivity assumption on $\mathfrak{a}$. By Hölder's inequality, Proposition 5.1(i) and Proposition 5.2(i), the first term in the right-hand side of (54) is majorated by $C(\operatorname{reg}(\mathfrak{T})) \times\|f\|_{L p^{\prime}} \times\left\|\nabla^{\mathfrak{T}} w^{\overline{\mathfrak{T}}}, \Delta t\right\|_{L^{p}}$. Finally, the last term in (54) is upper bounded by a constant times $m_{\Omega} \int_{-\left\|u_{0}\right\|_{L^{\infty}}}^{\| u_{L^{\infty}}} A(s) d s$. Hence, (ii) follows.

(iii) We proceed as in (ii), multiplying equations (33) by $u^{\mathfrak{\tau}, n}$ instead of $A\left(u^{\mathfrak{T}, n}\right)$. As in (54) above, taking $\theta=I d, \psi \equiv 1$, applying Proposition 4.2 instead of Proposition 4.1 neglecting the nonnegative terms on the left-hand side, we get

$$
\begin{aligned}
& \sum_{n=1}^{N} \Delta t\left(I_{\mathrm{Id}}\left[u^{\mathfrak{M}, n}, 1\right]+I_{\mathrm{Id}}^{*}\left[u^{\mathfrak{M} *, n}, 1\right]\right) \\
& \quad \leq \sum_{n=1}^{N} \Delta t \llbracket \mathbb{P}^{\mathfrak{T}}\left(\mathbb{S}^{\Delta t}[\mathcal{S}]\right)^{n}, u^{\mathfrak{T}, n} \rrbracket+\llbracket \frac{1}{2}\left(u^{\mathfrak{T}, 0}\right)^{2}, 1^{\mathfrak{T}} \rrbracket .
\end{aligned}
$$

Using the $L^{\infty}$ estimate (i) of the present proposition together with Proposition 5.1 (i), we finally get (iii) with the constant

$$
C=C(\operatorname{reg}(\mathfrak{T})) \times M \times\|\mathcal{S}\|_{L^{1}}+\frac{1}{2} m_{\Omega} \times\left(\left\|u_{0}\right\|_{L^{\infty}}\right)^{2} .
$$


(iv) We adapt to the discrete framework the calculation that led to estimate (8) in the proof of Theorem 2.1(ii). Denote by $J(\Delta), J^{*}(\Delta)$, respectively, the integrals

$$
\begin{aligned}
& \int_{Q}\left|u^{\mathfrak{M}, \Delta t}(t+\Delta, x)-u^{\mathfrak{M}, \Delta t}(t, x)\right|\left|A\left(u^{\mathfrak{m}, \Delta t}\right)(t+\Delta, x)-A\left(u^{\mathfrak{m}, \Delta t}\right)(t, x)\right|, \\
& \int_{Q}\left|u^{\mathfrak{M}^{*}, \Delta t}(t+\Delta, x)-u^{\mathfrak{M}^{*}, \Delta t}(t, x)\right|\left|A\left(u^{\mathfrak{M}^{*}, \Delta t}\right)(t+\Delta, x)-A\left(u^{\mathfrak{M}^{*}, \Delta t}\right)(t, x)\right| .
\end{aligned}
$$

Let us first take $k \in\{1, \ldots, N\}$ and estimate the quantity

$$
J_{0}(k):=\sum_{n=k+1}^{N} \Delta t \llbracket u^{\mathfrak{T}, n}-u^{\mathfrak{T},(n-k)}, A\left(u^{\mathfrak{T}, n}\right)-A\left(u^{\mathfrak{T},(n-k)}\right) \rrbracket .
$$

To do this, for $n=(k+1), \ldots, N$ we take the sum in $i$ from $(n-k+1)$ to $n$ of equations (33) and make the scalar product $\llbracket \cdot$, . $\rrbracket$ with the discrete functions $v^{\mathfrak{\tau}, n}$, where $v^{\overline{\mathfrak{s}}, n}:=A\left(u^{\overline{\mathfrak{s}}, n}\right)-A\left(u^{\overline{\mathfrak{s}},(n-k)}\right) \in \mathbf{R}_{0}^{\overline{\mathfrak{s}}}$ for $n=(k+1), \ldots, N$. Summing in $n$ and assigning $v^{\bar{s}}, n=0$ for $n=1, \ldots, k$ and $n=(N+1), \ldots,(N+k-1)$, we get

$$
\begin{aligned}
& \frac{J_{0}(k)}{\Delta t}=\sum_{n=k+1}^{N} \llbracket u^{\mathfrak{T}, n}-u^{\mathfrak{T},(n-k)}, v^{\mathfrak{T}, n} \rrbracket \\
&=\sum_{n=k+1}^{N} \Delta t \llbracket \sum_{i=n-k+1}^{n} \frac{u^{\mathfrak{T}, i}-u^{\mathfrak{T},(i-1)}}{\Delta t}, v^{\mathfrak{T}, n} \rrbracket \\
&=\sum_{i=2}^{N} \sum_{\min \{k, N-i+1\}} \sum_{j=\max \{1, k-i+2\}}^{N} \Delta t \llbracket \frac{u^{\mathfrak{T}, i}-u^{\mathfrak{T},(i-1)}}{\Delta t}, v^{\mathfrak{T},(i+j-1)} \rrbracket \\
&=\sum_{j=1}^{k} \sum_{i=2}^{N} \Delta t \llbracket-\left(\operatorname{div}_{c} \mathfrak{f}\right)^{\mathfrak{T}}\left[u^{\overline{\mathfrak{s}}, i}\right]+\operatorname{div}^{\mathfrak{T}}\left[\mathfrak{a}\left(\nabla^{\mathfrak{T}} w^{\overline{\mathfrak{s}}, i}\right)\right] \\
&-\mathcal{P}^{\mathfrak{T}}\left[w^{\overline{\mathfrak{s}}, i}\right]+\mathbb{P}^{\mathfrak{T}}\left(\mathbb{S}^{\Delta t}[\mathcal{S}]\right)^{i}, v^{\mathfrak{T},(i+j-1)} \rrbracket .
\end{aligned}
$$

We claim that the right-hand side of (55) is bounded by a constant independent of $h$. Indeed, for each $j=1, \ldots, k$, define $z_{j}^{\overline{\mathfrak{s}}, i}=v^{\overline{\mathfrak{s}},(i+j-1)}, i=1, \ldots, N$. First, from the property (ii) of the present proposition and from formula (42) we deduce

$$
\left\|\nabla^{\mathfrak{T}} z_{j}^{\overline{\mathfrak{s}}, \Delta t}\right\|_{L^{p}} \leq C, \quad \sum_{i=1}^{N} \Delta t \llbracket \mathcal{P}^{\overline{\mathfrak{T}}}\left[z_{j}^{\mathfrak{\mathcal { T }}, i}\right], z_{j}^{\mathfrak{\tau}, i} \rrbracket \leq C, \quad \text { for all } j=1, \ldots, k .
$$

In the sequel, we will omit the dependency of the entries of $z_{j}^{\overline{\mathfrak{s}}, \Delta t}$ on $j$.

By definition of $\left(\operatorname{div}_{c} \mathfrak{f}\right)^{\mathfrak{T}}[\cdot]$, taking into account that $z_{j}^{\frac{\pi}{\mathfrak{T}}, n} \in \mathbf{R}_{0}^{\overline{\mathfrak{z}}}$ and using summation-by-parts, we deduce that for all $j=1, \ldots, k$,

$$
\begin{aligned}
J_{1, j}:=\left|\sum_{i=1}^{N} \Delta t \llbracket(\operatorname{div} c \mathfrak{f})^{\mathfrak{T}}\left[u^{\overline{\mathfrak{z}}, i}\right], z_{j}^{\mathfrak{T}, i} \rrbracket\right| \\
=\mid \sum_{i=1}^{N} \Delta t\left(\frac{1}{d} \sum_{K \mid L \in \mathcal{E}} m_{K \mid L} g_{K, L}\left(u_{K}^{i}, u_{L}^{i}\right)\left(z_{K}^{i}-z_{L}^{i}\right)\right. \\
\left.\quad+\frac{d-1}{d} \sum_{K^{*} \mid L^{*} \in \mathcal{E}^{*}} m_{K^{*} \mid L^{*}} g_{K^{*}, L^{*}}\left(u_{K^{*}}^{i}, u_{L^{*}}^{i}\right)\left(z_{K^{*}}^{i}-z_{L^{*}}^{i}\right)\right) \mid .
\end{aligned}
$$


Since by (i), $u^{\mathfrak{m}, \Delta t}, u^{\mathfrak{m}^{*}, \Delta t}$ are bounded by $M$, using property (28) (d) we bound all values of $g_{K \mid L}, g_{K^{*} L^{*}}$ above by $C \omega_{M}(M)$. It follows by Remark 3.3 that

$$
\frac{\left|z_{K}^{i}-z_{L}^{i}\right|}{d_{K L}}+\frac{\left|z_{K^{*}}^{i}-z_{L^{*}}^{i}\right|}{d_{K^{*} L^{*}}} \leq\left|\nabla_{S} z^{\overline{\mathrm{s}}, i}\right|
$$

where $S=S_{K^{*}, L^{*}}^{K, L}$. Hence

$$
J_{1, j} \leq C(d-1) \omega_{M}(M) \sum_{i=1}^{N} \Delta t \sum_{S \in \mathfrak{G}} m_{S}\left|\nabla_{S} z^{\overline{\mathfrak{s}}, i}\right| \leq \text { const }\left\|\nabla^{\mathfrak{T}} z_{j}^{\overline{\mathfrak{s}}, \Delta t}\right\|_{L^{1}} .
$$

Using (56), we can uniformly bound $J_{1, j}$. Further, by Proposition 4.1 and the Hölder inequality,

$$
\begin{aligned}
& J_{2, j}:=\mid \sum_{i=1}^{N} \Delta t \llbracket\left[\operatorname{div}^{\mathfrak{T}}\left[\mathfrak{a}\left(\nabla^{\mathfrak{T}} w^{\overline{\mathfrak{T}}, i}\right)\right], z_{j}^{\mathfrak{T}, i} \rrbracket \mid\right. \\
& =\mid \sum_{i=1}^{N} \Delta t\left\{\left\{\mathfrak{a}\left(\nabla^{\mathfrak{T}} w^{\overline{\mathfrak{s}}, i}\right), \quad \nabla^{\mathfrak{T}} z_{j}^{\overline{\mathfrak{s}}, i}\right\} \mid \leq\left\|\mathfrak{a}\left(\nabla^{\mathfrak{T}} w^{\overline{\mathfrak{s}}, \Delta t}\right)\right\|_{L^{p^{\prime}}}\left\|\nabla^{\mathfrak{\mathfrak { T }}} z_{j}^{\overline{\mathfrak{s}}, \Delta t}\right\|_{L^{p}} .\right.
\end{aligned}
$$

Using the growth assumption on $\mathfrak{a}$ together with (56) and (ii) of the present lemma, we can uniformly bound $J_{2, j}$. Next, by (42) and the Cauchy-Schwarz inequality,

$$
\begin{aligned}
J_{3, j} & :=\left|\sum_{i=1}^{N} \Delta t \llbracket \mathcal{P}^{\mathfrak{T}}\left[w^{\overline{\mathfrak{s}}, i}\right], z_{j}^{\mathfrak{T}, i} \rrbracket\right| \\
\leq & \frac{d-1}{d} \sum_{i=1}^{N} \Delta t \sum_{K \in \overline{\mathfrak{M}}, K^{*} \in \overline{\mathfrak{M}^{*}}} m_{K \cap K^{*}} \frac{\left|w_{K}^{i}-w_{K^{*}}^{i}\right|}{\sqrt{\operatorname{size}(\mathfrak{T})}} \frac{\left|z_{K}^{i}-z_{K^{*}}^{i}\right|}{\sqrt{\operatorname{size}(\mathfrak{T})}} \\
\leq & \frac{d-1}{d}\left(\sum_{i=1}^{N} \Delta t \sum_{K \in \overline{\mathfrak{M}}, K^{*} \in \overline{\mathfrak{M}^{*}}} m_{K \cap K^{*}} \frac{\left|w_{K}^{i}-w_{K^{*}}^{i}\right|^{2}}{\operatorname{size}(\mathfrak{T})}\right)^{1 / 2} \\
& \times\left(\sum_{i=1}^{N} \Delta t \sum_{K \in \overline{\mathfrak{M}}, K^{*} \in \overline{\mathfrak{M}^{*}}} m_{K \cap K^{*}} \frac{\left|z_{K}^{i}-z_{K^{*}}^{i}\right|^{2}}{\operatorname{size}(\mathfrak{T})}\right)^{1 / 2} \\
= & \left(\sum_{i=1}^{N} \Delta t \llbracket \mathcal{P}^{\mathfrak{T}}\left[w^{\overline{\mathfrak{s}}, i}\right], w^{\mathfrak{T}, i} \rrbracket\right)^{1 / 2}\left(\sum_{i=1}^{N} \Delta t \llbracket \mathcal{P}^{\mathfrak{T}}\left[z_{j}^{\overline{\mathfrak{z}}, i}\right], z_{j}^{\mathfrak{s}, i} \rrbracket\right)^{1 / 2} .
\end{aligned}
$$

Using again (56) and (ii) of the present proposition, we can uniformly bound $J_{3, j}$. Finally, like in (54), we have

$$
J_{4, j}:=\left|\sum_{i=1}^{N} \Delta t \llbracket \mathbb{P}^{\mathfrak{I}}\left(\mathbb{S}^{\Delta t}[\mathcal{S}]\right)^{i}, z_{j}^{\mathfrak{s}, i} \rrbracket\right| \leq C(\operatorname{reg}(\mathfrak{T})) \times\|\mathcal{S}\|_{L^{p^{\prime}}} \times\left\|\nabla^{\mathfrak{T}} z_{j}^{\overline{\mathfrak{T}}, \Delta t}\right\|_{L^{p}},
$$

which is also uniformly bounded, thanks to (56). Gathering the estimates above, we conclude

$$
J_{0}(k) \leq \Delta t \sum_{j=1}^{k}\left(J_{1, j}+J_{2, j}+J_{3, j}+J_{4, j}\right) \leq C k \Delta t
$$


Using the definition of $\llbracket \ldots \rrbracket$ and the $L^{\infty}$ estimate on $u^{\mathfrak{m}, \Delta t}$, cf. (i), we get

$$
\frac{1}{d} J(k \Delta t)+\frac{d-1}{d} J^{*}(k \Delta t) \leq J_{0}(k)+\int_{(N-k) \Delta t}^{N \Delta t} m_{\Omega} M \max \{ \pm A( \pm M)\} \leq C k \Delta t .
$$

Now let $0<\Delta<T$. We have $\Delta / \Delta t=(k-1)+\alpha$ for some $k \in\{1, \ldots, N\}$ and $\alpha \in[0,1)$. Since $u^{\mathfrak{m}, \Delta t}$ is piecewise constant in $t$ with step $\Delta t$, we have

$$
\begin{aligned}
J(\Delta) & =J((k-1) \Delta t+\alpha \Delta t) \leq \alpha J(k \Delta t)+(1-\alpha) J((k-1) \Delta t) \\
& \leq \alpha C k \Delta t+(1-\alpha) C(k-1) \Delta t \leq C((k-1)+\alpha) \Delta t=C \Delta .
\end{aligned}
$$

From (58), together with the calculation used to pass from (8) to (9) (cf. the proof of Theorem 2.1), we deduce the required estimate

$$
\int_{Q}\left|A\left(u^{\mathfrak{m}, \Delta t}\right)(t+\Delta, x)-A\left(u^{\mathfrak{m}, \Delta t}\right)(t, x)\right| \leq \omega_{A}(\Delta), \quad \Delta>0 .
$$

Similarly, time translates of $A\left(u^{\mathfrak{M}^{*}, \Delta t}\right)$ are controlled with $J^{*}(k \Delta t)$ in (57).

\subsection{Existence of discrete solutions.}

Proposition 6.2. Let $\mathfrak{T}$ be a double mesh of $\Omega$ and $\Delta t>0$. There exists a solution $u^{\overline{2}, \overline{\Delta t}}$ of the finite volume scheme (33), (34), (35).

Proof. First note that it is sufficient to prove existence of solutions $u_{\rho}^{\overline{\tilde{s}}, \overline{\Delta t}}$ to (33), (34), (35) with $A(\cdot)$ replaced by a strictly increasing function $A_{\rho}(\cdot)$. Indeed, using the $L^{\infty}$ estimate (i) of Proposition 6.1 which is independent of the choice of $A(\cdot)$, we get compactness of $u_{\rho}^{\overline{\boldsymbol{\tau}}, \overline{\Delta t}}$ in the finite-dimensional space $\mathbf{R}^{(N+1) \times \overline{\boldsymbol{\tau}}}$. Choosing a sequence of strictly increasing functions $A_{\rho}$ that converges to $A$ uniformly on all compact of $\mathbf{R}$, we pass to the limit in the scheme (33), (34), (35) written for (a subsequence of $) A_{\rho}(\cdot)$ and $u_{\rho}^{\bar{\Sigma}, \overline{\Delta t}}$ and obtain existence for general $A(\cdot)$.

Let us now assume that $A(\cdot)$ is invertible and rewrite the scheme in terms of $w^{\overline{\mathfrak{s}}, \overline{\Delta t}}$ with $u^{\overline{\mathfrak{s}}, \overline{\Delta t}}=A^{-1}\left(w^{\overline{\mathfrak{s}}, \overline{\Delta t}}\right)$. The existence of $w^{\mathfrak{s}, n}$ is shown by induction on $n=0, \ldots, N$. For $n=0$, solution is given by (35). Assume that $w^{\mathfrak{\tau},(n-1)}$ exists. Choose $\llbracket \cdot, \cdot]$ as the scalar product on $\mathbf{R}^{\mathfrak{T}}$. We are looking for a solution $w^{\mathfrak{T}, n}$ to $L\left[w^{\mathfrak{\tau}, n}\right]=0$, where the operator $L$ is given by

$$
\begin{aligned}
L: z^{\mathfrak{T}} \in \mathbf{R}^{\mathfrak{T}} \mapsto \frac{A^{-1}\left(z^{\mathfrak{T}}\right)-A^{-1}\left(w^{\mathfrak{T},(n-1)}\right)}{\Delta t}+\left(\operatorname{div}_{c} \mathfrak{f}\right)^{\mathfrak{T}}\left[A^{-1}\left(z^{\overline{\mathfrak{T}}}\right)\right] \\
-\operatorname{div}^{\mathfrak{\mathfrak { T }}}\left[\mathfrak{a}\left(\nabla^{\mathfrak{T}} z^{\overline{\mathfrak{x}}}\right)\right]+\mathcal{P}^{\mathfrak{T}}\left[z^{\overline{\mathfrak{T}}}\right]-\mathbb{P}^{\mathfrak{\mathfrak { T }}}\left(\mathbb{S}^{\Delta t}[\mathcal{S}]\right)^{n} .
\end{aligned}
$$

By Proposition 4.3 with $\theta=\operatorname{Id}$ and $\psi \equiv 1$, by Proposition 4.1 and by Lemma 4.1 . there exists a constant $C=C\left(\left\|w^{\mathfrak{T}, n-1}\right\|_{\mathbf{R}^{\mathfrak{T}}},\left\|\mathbb{P}^{\mathfrak{T}}\left(\mathbb{S}^{\Delta t}[\mathcal{S}]\right)^{n}\right\|_{\mathbf{R}^{\mathfrak{T}}}, \Delta t\right)$ such that

$$
\llbracket L\left[z^{\mathfrak{T}}\right], z^{\mathfrak{T}} \rrbracket \geq\left\{\left\{\mathfrak{a}\left(\nabla^{\mathfrak{T}} z^{\overline{\mathfrak{T}}}\right), \nabla^{\mathfrak{T}} z^{\overline{\mathfrak{T}}}\right\}-C\left\|z^{\mathfrak{T}}\right\|_{\mathbf{R}^{\mathfrak{T}}} .\right.
$$

By the coercivity assumption on $\mathfrak{a}$ and by Proposition 5.2 (i) we have

$$
\left\{\left\{\mathfrak{a}\left(\nabla^{\mathfrak{T}} z^{\overline{\mathfrak{T}}}\right), \nabla^{\mathfrak{T}} z^{\overline{\mathfrak{T}}}\right\} \geq \text { const }\left\|\nabla^{\mathfrak{T}} z^{\overline{\mathfrak{T}}}\right\|_{L^{p}}^{p} \geq \mathrm{const}\left(\left\|z^{\mathfrak{M}}\right\|_{L^{p}}^{p}+\left\|z^{\mathfrak{M}^{*}}\right\|_{L^{p}}^{p}\right) .\right.
$$

Because the right-hand side of (59) is equivalent to $\left(\left\|z^{\mathfrak{T}}\right\|_{\mathbf{R}^{\mathfrak{T}}}\right)^{p}$, we conclude that $\llbracket L\left[z^{\mathfrak{T}}\right], z^{\mathfrak{T}} \rrbracket \geq 0$ for $\left\|z^{\mathfrak{T}}\right\|_{\mathbf{R}^{\mathfrak{T}}}$ sufficiently large. The existence of $w^{\mathfrak{s}, n}$ follows by the standard Brouwer fixed point argument (see [64, Lemme 4.3]). 
We point out that the uniqueness and, more generally, continuous dependency of the discrete solutions on the data can be established as well (see [48, 49, 11] for results of that sort). However, in view of the convergence result of Theorem 7.1 and the well-posedness of the continuous problem, we view these questions to be of less importance.

\subsection{Discrete entropy inequalities.}

Proposition 6.3. Let $\mathfrak{T}$ be a double mesh of $\Omega$ and $\Delta t>0$. Consider a solution $u^{\bar{\tau}, \overline{\Delta t}}$ to the scheme (33), (34), (35); recall that $w^{\overline{\mathrm{s}}, \overline{\Delta t}}=A\left(u^{\overline{\mathrm{s}}, \overline{\Delta t}}\right)$.

Let $\psi \in \mathcal{D}(\bar{Q}), \psi \geq 0 ;$ set $\psi^{\overline{\bar{x}}, \Delta t}=\mathbb{P}^{\overline{\bar{x}}} \circ \mathbb{S}^{\Delta t}[\psi]$. Let $\theta: \mathbf{R} \rightarrow \mathbf{R}$ be a nondecreasing function; assume that $\psi$ and $\theta$ are chosen so that (38) holds; assume that $\Delta t$ is small enough. Then

$$
\begin{aligned}
& -\llbracket \eta\left(u^{\mathfrak{T}, N}\right), \psi^{\mathfrak{T}, N} \rrbracket+\sum_{n=1}^{N-1} \Delta t \llbracket \eta\left(u^{\mathfrak{T}, n}\right), \frac{\psi^{\mathfrak{T},(n+1)}-\psi^{\mathfrak{T}, n}}{\Delta t} \rrbracket \\
& +\sum_{n=1}^{N} \Delta t \llbracket \mathfrak{q}\left(u^{\mathfrak{T}, n}\right),(\nabla \psi)^{\mathfrak{I}, n} \rrbracket-\sum_{n=1}^{N} \Delta t\left\{\left\{k\left(\nabla^{\mathfrak{T}} w^{\overline{\mathfrak{x}}, n}\right) \nabla^{\mathfrak{I}} \widetilde{A}_{\theta}\left(w^{\overline{\mathfrak{s}}, n}\right), \nabla^{\mathfrak{T}} \psi^{\overline{\mathfrak{s}}, n}\right\}\right. \\
& +\llbracket \eta\left(u^{\mathfrak{T}, 0}\right), \psi^{\mathfrak{T}, 1} \rrbracket+\sum_{n=1}^{N} \Delta t \llbracket \mathbb{P}^{\mathfrak{T}}\left(\mathbb{S}^{\Delta t}[f]\right)^{n}, \theta\left(u^{\mathfrak{T}, n}\right) \psi^{\mathfrak{T}, n} \rrbracket \\
& \geq \frac{d-1}{d} \sum_{n=1}^{N} \Delta t \sum_{K \in \overline{\mathfrak{M}}, K^{*} \in \overline{\mathfrak{M}^{*}}} m_{K \cap K^{*}} \theta\left(u_{K}\right) \frac{\left(w_{K}^{n}-w_{K^{*}}^{n}\right)\left(\psi_{K}^{n}-\psi_{K^{*}}^{n}\right)}{\operatorname{size}(\mathfrak{T})} \\
& +R_{\theta}\left[u^{\mathfrak{M}, n}, \psi^{n}\right]+R_{\theta}^{*}\left[u^{\mathfrak{M}^{*}, n}, \psi^{n}\right]
\end{aligned}
$$

where $A_{\theta}(\cdot), \widetilde{A}_{\theta}(\cdot)$ and $\eta(\cdot), \mathfrak{q}(\cdot), R_{\theta}[\cdot, \cdot], R_{\theta}^{*}[\cdot, \cdot]$ are introduced in Definition 2.1 and in Proposition 4.3 , respectively.

Moreover, with the specific choice $\theta \equiv 1$ and $\psi \in \mathcal{D}([0, T) \times \Omega)$, there holds

$$
\begin{aligned}
& \sum_{n=1}^{N-1} \Delta t \llbracket u^{\mathfrak{T}, n}, \frac{\psi^{\mathfrak{T},(n+1)}-\psi^{\mathfrak{T}, n}}{\Delta t} \rrbracket+\llbracket u^{\mathfrak{T}, 0}, \psi^{\mathfrak{T}, 1} \rrbracket+\sum_{n=1}^{N} \Delta t \llbracket \mathfrak{f}\left(u^{\mathfrak{T}, n}\right),(\nabla \psi)^{\mathfrak{T}, n} \rrbracket \\
& -\sum_{n=1}^{N} \Delta t\left\{\left\{k\left(\nabla^{\mathfrak{T}} w^{\overline{\mathfrak{s}}, n}\right) \nabla^{\mathfrak{T}} w^{\overline{\mathfrak{s}}, n}, \nabla^{\mathfrak{T}} \psi^{\overline{\mathfrak{s}}, n}\right\}+\sum_{n=1}^{N} \Delta t \llbracket \mathbb{P}^{\mathfrak{T}}\left(\mathbb{S}^{\Delta t}[\mathcal{S}]\right)^{n}, \psi^{\mathfrak{s}, n} \rrbracket\right. \\
& =\frac{d-1}{d} \sum_{n=1}^{N} \Delta t \sum_{K \in \overline{\mathfrak{M}}, K^{*} \in \overline{\mathfrak{M}^{*}}} m_{K \cap K^{*}} \frac{\left(w_{K}^{n}-w_{K^{*}}^{n}\right)\left(\psi_{K}^{n}-\psi_{K^{*}}^{n}\right)}{\operatorname{size}(\mathfrak{T})} \\
& +R_{1}\left[u^{\mathfrak{M}, n}, \psi^{n}\right]+R_{1}^{*}\left[u^{\mathfrak{M}^{*}, n}, \psi^{n}\right] .
\end{aligned}
$$

Finally, with the specific choices $\theta \equiv A$ and $\psi \equiv \zeta(t)$, where $\zeta \in \mathcal{D}([0, T))$ is a nonnegative, nonincreasing function with $\zeta(t) \equiv 1$ for small $t$, we have with 
$B(z)=\int_{0}^{z} A(s) d s$

$$
\begin{aligned}
& \sum_{n=1}^{N-1} \Delta t \llbracket B\left(u^{\mathfrak{T}, n}\right), \frac{\zeta^{\mathfrak{T},},(n+1)-\zeta^{\mathfrak{T}, n}}{\Delta t} \rrbracket+\llbracket B\left(u^{\mathfrak{T}, 0}\right), 1^{\mathfrak{T}} \rrbracket \\
& +\sum_{n=1}^{N} \Delta t \llbracket \mathbb{P}^{\mathfrak{T}}\left(\mathbb{S}^{\Delta t}[\mathcal{S}]\right)^{n}, w^{\mathfrak{T}, n} \zeta^{\mathfrak{T}, n} \rrbracket \\
& \quad \geq \sum_{n=1}^{N} \Delta t\left\{k\left(\nabla^{\mathfrak{T}} w^{\overline{\mathfrak{s}}, n}\right) \nabla^{\mathfrak{T}} w^{\overline{\mathfrak{s}}, n}, \nabla^{\mathfrak{T}} w^{\overline{\mathfrak{s}}, n} \zeta^{\overline{\mathfrak{s}}, n}\right\} .
\end{aligned}
$$

Proof. Inequality (60) follows by an application of Lemma 4.2. Proposition 4.3 Proposition 4.2 and Lemma 4.1. Note that in (60), we have neglected the positive terms $I_{\theta}\left[u^{\mathfrak{m}, n}, \psi^{n}\right], I_{\theta}^{*}\left[u^{\mathfrak{M}^{*}, n}, \psi^{n}\right]$. In (61) the corresponding terms are zero because $\theta \equiv 1$, and we use the equality of Proposition 4.1 instead of the inequality of Proposition 4.2. Also notice that the term with $\psi^{\mathfrak{T}, N}$ in Lemma 4.2 disappears because $\Delta t$ is small and $\psi$ vanishes in a neighborhood of $t=T$. Finally, in (62) we have treated $A\left(u^{\bar{\Sigma}}, \Delta t\right) \zeta^{\overline{\mathbf{s}}, \Delta t}$ as a mere test function by applying Proposition 4.1 on the right-hand side, but we have used Lemma 4.2. Proposition 4.3 and the choice of the constant in $x$ function $\psi^{\bar{s}, \Delta t}$ to deal with the remaining terms.

6.4. Control of the remainder terms in Proposition 6.3. For all $\psi \in \mathcal{D}(\bar{Q})$, the terms on the right-hand side of (60), (61) coming from the penalization operator vanish as $h \rightarrow 0$. Indeed, using the estimates of Proposition 6.1(i),(ii), the CauchySchwarz inequality, Proposition[5.1(iv), and the boundedness of $\theta$ on $[-M, M]$, we obtain

$$
\begin{aligned}
& \left|\sum_{n=1}^{N} \Delta t \sum_{K \in \overline{\mathfrak{M}}, K^{*} \in \overline{\mathfrak{M}^{*}}} m_{K \cap K^{*}} \theta\left(u_{K}\right) \frac{\left(w_{K}^{n}-w_{K^{*}}^{n}\right)\left(\psi_{K}^{n}-\psi_{K^{*}}^{n}\right)}{\operatorname{size}(\mathfrak{T})}\right| \\
& \quad \leq C\left(\sum_{n=1}^{N} \Delta t \llbracket \mathcal{P}^{\mathfrak{T}}\left[w^{\overline{\mathfrak{s}}, n}\right], w^{\mathfrak{T}, n} \rrbracket\right)^{1 / 2}\left(\sum_{n=1}^{N} \Delta t \sum_{K \in \overline{\mathfrak{M}}, K^{*} \in \overline{\mathfrak{M}^{*}}} m_{K \cap K^{*}} \frac{\left|\psi_{K}^{n}-\psi_{K^{*}}^{n}\right|^{2}}{\operatorname{size}(\mathfrak{T})}\right)^{1 / 2} \\
& \quad \leq C\|\nabla \psi\|_{L^{\infty}} \times \operatorname{size}(\mathfrak{T}) .
\end{aligned}
$$

Let us show that the terms $R_{\theta}\left[u^{\mathfrak{m}}, \psi\right], R_{\theta}^{*}\left[u^{\mathfrak{m} *}, \psi\right]$ in (60), (61) (which are defined in Proposition 4.3) vanish as $h \rightarrow 0$. This holds true thanks to their upper bounds in terms the quantities $I_{\mathrm{Id}}\left[u^{\mathfrak{M}}, 1\right], I_{\mathrm{Id}}^{*}\left[u^{\mathfrak{M}^{*}}, 1\right]$, quantities which are controlled by means of Proposition 6.1(iii) (known as the "weak BV estimate", cf. [48]).

Proposition 6.4. Let $_{K, L} \in C\left(\mathbf{R}^{2}\right)$ be a function with properties (28) (a),(d). For $a, b \in \mathbf{R}$, consider

$$
\begin{gathered}
I_{\mathrm{Id}}^{K \mid L}(a, b)=\int_{a}^{b}\left(g_{K, L}(s, s)-g_{K, L}(a, b)\right) d s \\
R_{K}^{K \mid L}(a, b)=\left|g_{K, L}(a, a)-g_{K, L}(a, b)\right|, \quad R_{L}^{K \mid L}(a, b)=\left|g_{K, L}(b, b)-g_{K, L}(a, b)\right| .
\end{gathered}
$$

There exists a continuous strictly increasing convex function $\Pi_{M}: \mathbf{R}^{+} \rightarrow \mathbf{R}^{+}$ that only depends on $C$ and $\omega_{M}(\cdot)$ in (28) (d) such that $\Pi_{M}(0)=0, \Pi_{M}^{\prime}(0)=0$ and the following bounds hold:

$$
\left\{\begin{array}{l}
R_{K}^{K \mid L}(a, b) \leq \Pi_{M}^{-1}\left(I_{\mathrm{Id}}^{K \mid L}(a, b)\right), \\
R_{L}^{K \mid L}(a, b) \leq \Pi_{M}^{-1}\left(I_{\mathrm{Id}}^{K \mid L}(a, b)\right),
\end{array} \quad \text { for all } a, b \in[-M, M] .\right.
$$


The proof is based upon the following generalization of [48, Lemma 4.5].

Lemma 6.1. Let $g \in C([a, b])$ be a nondecreasing function equipped with a modulus of continuity $\omega$. Then

$$
\int_{a}^{b}(g(s)-g(a)) d s \geq \int_{0}^{g(b)-g(a)} \omega^{-1}(r) d r .
$$

Proof. Set $\delta=\omega^{-1}(g(b)-g(a))$. Since $|g(b)-g(s)| \leq \omega(b-s)$ and $g$ is nondecreasing, we have

$$
g(s) \geq \begin{cases}g(b)-\omega(b-s), & b-\delta \leq s \leq b \\ g(a), & a \leq s \leq b-\delta .\end{cases}
$$

Hence setting $z=b-s$, integrating by parts, and setting $r=\omega(z)$, we deduce

$$
\begin{aligned}
\int_{a}^{b}(g(s)-g(a)) d s & \geq \int_{b-\delta}^{b}(g(b)-g(a)-\omega(b-s)) d s \\
& =\delta \omega(\delta)-\int_{0}^{\delta} \omega(z) d z=\int_{0}^{\delta} z d \omega(z)=\int_{0}^{\omega(\delta)} \omega^{-1}(r) d r .
\end{aligned}
$$

Proof of Proposition 6.4. Consider the case $a \leq b$. By (28) (a), we have

$$
I_{\mathrm{Id}}^{K \mid L}(a, b)=\int_{a}^{b}\left(g_{K, L}(s, s)-g_{K, L}(a, b)\right) d s \geq \int_{a}^{b}\left(g_{K, L}(s, b)-g_{K, L}(a, b)\right) d s ;
$$

applying Lemma 6.1 to $g(\cdot)=g_{K, L}(\cdot, b)$ and recalling (28) (d), we deduce

$$
I_{\mathrm{Id}}^{K \mid L}(a, b) \geq \int_{0}^{g_{K, L}(b, b)-g_{K, L}(a, b)}\left(C \omega_{M}\right)^{-1}(r) d r=\int_{0}^{R_{L}^{K \mid L}(a, b)}\left(C \omega_{M}\right)^{-1}(r) d r .
$$

Thus in order to estimate $R_{L}^{K \mid L}(a, b)$ as in (63), it is sufficient to take the function $\Pi_{M}: R \in \mathbf{R}^{+} \mapsto \int_{0}^{R}\left(C \omega_{M}\right)^{-1}(r) d r$. Clearly, $\Pi_{M}$ is continuous, strictly increasing, convex, $\Pi_{M}(0)=0$, and $\Pi_{M}^{\prime}(0)=0$.

The other estimate in (63) is obtained in the same way, and the case $a>b$ is obtained by symmetry.

Corollary 6.1. (i) Consider $I_{\mathrm{Id}}\left[u^{\mathfrak{M}}, 1\right]$ defined as in (45), (46) with $\theta=\mathrm{Id}$, and $\psi \equiv 1$. For general nondecreasing $\theta(\cdot)$ and general $\psi \in \mathcal{D}(\bar{\Omega})$, consider $R_{\theta}\left[u^{\mathfrak{M}}, \psi\right]$ defined in (47),(48). Assume $\left\|u^{\mathfrak{M}}\right\|_{\infty} \leq M$. Let $\Pi_{M}$ be the function given in Proposition 6.4. Let $\Pi_{M}^{*}$ be the conjugate convex function of $\Pi_{M}$. Then

$$
\begin{aligned}
& \left|R_{\theta}\left[u^{\mathfrak{M}}, \psi\right]\right| \\
& \leq 2\|\theta\|_{C([-M, M])} \inf _{\alpha>0}\left(\frac{\operatorname{size}(\mathfrak{T})}{\alpha} I_{\mathrm{Id}}\left[u^{\mathfrak{M}}, 1\right]+\frac{C}{\alpha} \Pi_{M}^{*}\left(2 \alpha \max _{K \in \mathfrak{M}, L \in \mathcal{N}(K)} \frac{\left|\psi_{K}-\psi_{K \mid L}\right|}{d_{K, K \mid L}}\right)\right),
\end{aligned}
$$

where $C$ depends on $\operatorname{reg}(\mathfrak{T}), d$ and $\Omega$.

(ii) Assume we are given a sequence of meshes $\boldsymbol{T}$ with size $(\boldsymbol{T}) \rightarrow 0$ and time steps $\Delta t \rightarrow 0$. Let $u^{\mathfrak{T}, \Delta t}$ be the corresponding discrete functions such that $\left\|u^{\mathfrak{M}, \Delta t}\right\|_{\infty} \leq M$ and $\sum_{n=1}^{N} \Delta t I_{\mathrm{Id}}\left[u^{\mathfrak{M}, n}, 1\right] \leq C$ uniformly in $\mathfrak{T}, \Delta t$. Choose $\psi \in \mathcal{D}(\bar{Q})$ and take $\psi^{n}=\left(\mathbb{S}^{\Delta t}[\psi]\right)^{n}$. Then $\sum_{n=1}^{N} \Delta t R_{\theta}^{*}\left[u^{\overline{\mathfrak{T}}, n}, \psi^{n}\right] \rightarrow 0$ as $\operatorname{size}(\mathfrak{T}) \rightarrow 0$.

Analogous statements that involve $\sum_{n=1}^{N} \Delta t I_{\mathrm{Id}}^{*}\left[u^{\mathfrak{M}^{*}}, 1\right]$ and $\psi_{K^{*}}, \psi_{K^{*} \mid L^{*}}$ with $K^{*} \in \mathfrak{M}^{*}, L^{*} \in \mathcal{N}^{*}\left(K^{*}\right)$ hold for $\sum_{n=1}^{N} \Delta t R_{\theta}^{*}\left[u^{\mathfrak{M}^{*}}, \psi\right]$. 
Proof. (i) By (47) and Proposition 6.4, for all $\alpha>0$ we have

$$
\begin{aligned}
& \left|R_{\theta}\left[u^{\mathfrak{M}}, \psi\right]\right| \\
& \quad \leq 2\|\theta\|_{C([-M, M])} \sum_{K \in \mathfrak{M}, L \in \mathcal{N}(K)}\left(\frac{1}{\alpha} m_{K \mid L} d_{K, K \mid L}\right) \times \Pi_{M}^{-1}\left(I_{\mathrm{Id}}^{K \mid L}\right) \times\left(\alpha \frac{\left|\psi_{K}-\psi_{K \mid L}\right|}{d_{K, K \mid L}}\right) .
\end{aligned}
$$

Note that $d_{K L} \leq \operatorname{size}(\mathfrak{T})$. Further, even in the case the diamonds are not necessarily convex, the definition of $\operatorname{reg}(\mathfrak{T})$ permits to control the multiplicity of the covering of $\Omega$ by the convex envelopes of $K$ and $K \mid L, K \in \mathfrak{M}, L \in \mathcal{N}(K)$. Thus one can upper bound $\sum_{K \in \mathfrak{M}, L \in \mathcal{N}(K)} m_{K \mid L} d_{K, K \mid L}$ by $C(\operatorname{reg}(\mathfrak{T}), d) m_{\Omega}$. Applying the inequality $r s \leq$ $\Pi_{M}(r)+\Pi_{M}^{*}(s)$ on the right-hand side above, we deduce (64).

(ii) First notice that for all $\psi \in \mathcal{D}(\bar{Q})$, there exists $C>0$ such that

$$
\max _{n=1, \ldots, N, K \in \mathfrak{M}, L \in \mathcal{N}(K)} \frac{\left|\psi_{K}^{n}-\psi_{K \mid L}^{n}\right|}{d_{K, K \mid L}} \leq C, \quad \text { for all } h>0 .
$$

Applying (i) for each $n$ and summing over $n=1, \ldots, N$, we get

$$
\begin{aligned}
\sum_{n=1}^{N} \Delta t R_{\theta}^{*}\left[u^{\overline{\mathfrak{s}}, n}, \psi^{n}\right] & \leq C \inf _{\alpha>0}\left(\frac{\operatorname{size}(\mathfrak{T})}{\alpha} \sum_{n=1}^{N} \Delta t I_{\mathrm{Id}}\left[u^{\mathfrak{M}}, 1\right]+T \frac{1}{\alpha} \Pi_{M}^{*}(C \alpha)\right) \\
& \leq C \inf _{\alpha>0}\left(\frac{\operatorname{size}(\mathfrak{T})}{\alpha}+\frac{1}{\alpha} \Pi_{M}^{*}(C \alpha)\right),
\end{aligned}
$$

where $C$ stands for a generic constant independent of $h$.

We have $\left(\Pi_{M}\right)^{\prime}(0)=0$. Therefore

$$
\left(\Pi_{M}^{*}\right)^{\prime}(0)=\lim _{b \rightarrow 0} \inf _{a}\left(a-\frac{\Pi_{M}(a)}{b}\right) \leq \lim _{b \rightarrow 0}\left(b-\frac{\Pi_{M}(b)}{b}\right)=0 .
$$

Hence for all $C>0, \lim _{\alpha \rightarrow 0} \frac{1}{\alpha} \Pi_{M}^{*}(C \alpha)=0$. We deduce that the right-hand side of (65) tends to zero as $\operatorname{size}(\mathfrak{T}) \rightarrow 0$.

Remark 6.1. Notice that if $\mathfrak{f}$ is locally Lipschitz continuous, both $\Pi_{M}$ and $\Pi_{M}^{*}$ are quadratic; thus we can bound $\left|R_{\theta}\left[u^{\bar{\Sigma}}, \psi\right]\right|$ by Const $h^{\beta}$ for all $\beta<1 / 2$. Using the Hölder inequality instead of the Young inequality, one recovers the result of 48 with $\beta=1 / 2$. Whenever $\mathfrak{f}$ is locally Hölder continuous of order $\gamma \leq 1$, we find $\Pi_{M}^{*}(s)=$ Const $s^{1+\gamma}$. It follows that $\left|R_{\theta}\left[u^{\overline{\mathfrak{x}}}, \psi\right]\right| \leq$ Const $h^{\beta}$ with $\beta=\frac{\gamma}{\gamma+1}$, under the assumptions of Corollary 6.1(ii).

6.5. Approximate continuous entropy inequalities. Relying on Proposition 6.3. we now deduce the limiting (as $h \rightarrow 0$ ) entropy inequalities and the limiting weak formulation; one should notice that they continue to hold if we replace $\left(\eta_{c}^{ \pm}, \mathfrak{q}_{c}^{ \pm}\right)$ by regular "boundary" entropy-entropy flux pairs $\left(\eta_{c, \varepsilon}^{ \pm}, \mathfrak{q}_{c, \varepsilon}^{ \pm}\right)$.

Proposition 6.5. Consider a family of double meshes $\mathfrak{T}$ of $\Omega$ and associated time steps $\Delta t>0$, parametrized by $h=\max \{\operatorname{size}(\mathfrak{T}), \Delta t\}, h \rightarrow 0$. Assume that $\operatorname{reg}(\mathfrak{T})$ is uniformly bounded. Denote the corresponding discrete solutions of (33), (34), (35) by $u^{\overline{\bar{s}}, \overline{\Delta t}}$. Fix $\psi \in \mathcal{D}([0, T) \times \bar{\Omega}), \psi \geq 0$, and set $\psi^{\overline{\mathbf{s}}, \Delta t}=\mathbb{P}^{\overline{\mathbf{s}}} \circ \mathbb{S}^{\Delta t}[\psi]$. Fix $\theta$ as one of the functions $\eta_{c}^{ \pm}, c \in \mathbf{R}$. Assume either $(c, \psi) \in \mathbf{R}^{ \pm} \times \mathcal{D}([0, T) \times \bar{\Omega})$, or 
$(c, \psi) \in \mathbf{R} \times \mathcal{D}([0, T) \times \Omega)$. Then

$$
\begin{aligned}
\liminf _{h \rightarrow 0}\left(\int_{Q} \frac{1}{d}\left(\eta_{c}^{ \pm}\left(u^{\mathfrak{M}, \Delta t}\right)+(d-1) \eta_{c}^{ \pm}\left(u^{\mathfrak{M}^{*}, \Delta t}\right)\right) \partial_{t} \psi\right. \\
+\int_{Q} \frac{1}{d}\left(\mathfrak{q}_{c}^{ \pm}\left(u^{\mathfrak{M}, \Delta t}\right)+(d-1) \mathfrak{q}_{c}^{ \pm}\left(u^{\mathfrak{M}^{*}, \Delta t}\right)\right) \cdot \nabla \psi \\
\quad-\int_{Q} k\left(\nabla^{\mathfrak{\mathfrak { T }}} w^{\overline{\mathfrak{s}}, \Delta t}\right) \nabla^{\mathfrak{\mathfrak { T }}} \widetilde{A}_{\left(\eta_{c}^{ \pm}\right)^{\prime}}\left(w^{\overline{\mathfrak{s}}, \Delta t}\right) \cdot \nabla \psi \\
\quad+\int_{\Omega} \frac{1}{d}\left(\eta_{c}^{ \pm}\left(u^{\mathfrak{M}, 0}\right)+(d-1) \eta_{c}^{ \pm}\left(u^{\mathfrak{M} *}, 0\right)\right) \psi(0, \cdot) \\
\left.\quad+\int_{Q} \frac{1}{d}\left(\left(\eta_{c}^{ \pm}\right)^{\prime}\left(u^{\mathfrak{M}, \Delta t}\right)+(d-1)\left(\eta_{c}^{ \pm}\right)^{\prime}\left(u^{\mathfrak{M}^{*}, \Delta t}\right)\right) \mathcal{S} \psi\right) \geq 0 .
\end{aligned}
$$

Furthermore, if $\psi \in \mathcal{D}([0, T) \times \Omega)$, we have

$$
\begin{aligned}
& \lim _{h \rightarrow 0}\left(\int_{Q} \frac{1}{d}\left(u^{\mathfrak{M}, \Delta t}+(d-1) u^{\mathfrak{M}^{*}, \Delta t}\right) \partial_{t} \psi\right. \\
& +\int_{Q}\left(\frac{1}{d}\left(\mathfrak{f}\left(u^{\mathfrak{M}, \Delta t}\right)+(d-1) \mathfrak{f}\left(u^{\mathfrak{M} *}, \Delta t\right)\right)-k\left(\nabla^{\mathfrak{T}} w^{\overline{\mathfrak{s}}, \Delta t}\right) \nabla^{\mathfrak{T}} w^{\overline{\mathfrak{s}}, \Delta t}\right) \cdot \nabla \psi \\
& \left.+\int_{\Omega} \frac{1}{d}\left(u^{\mathfrak{m}, 0}+(d-1) u^{\mathfrak{M}^{*}, 0}\right) \psi(0, \cdot)+\int_{Q} \mathcal{S} \psi\right)=0 .
\end{aligned}
$$

Proof. By the choice of $(c, \psi)$, (38) holds. Thus, by Proposition 6.3, (60) and (61) hold; it suffices to develop these formulas using the definitions of $\mathbb{[} \cdot, \cdot],\{\{\cdot, \cdot\}$.

The second term in (60) rewrites exactly as the corresponding term in (66). Regarding the other terms on the left-hand side, we also use the uniform bound on $u^{\mathfrak{T}, n}$ in $L^{\infty}$, the uniform bound on $k\left(\nabla^{\mathfrak{T}} w^{\mathfrak{T}, \Delta t}\right) \nabla^{\mathfrak{T}} w^{\mathfrak{T}, \Delta t}$ in $\left(L^{p^{\prime}}(Q)\right)^{d}$, and the convergences

$$
\begin{aligned}
& \sum_{n=1}^{N} \sum_{K \in \mathfrak{M}} \frac{\psi_{K}^{(n+1)}-\psi_{K}^{n}}{\Delta t} \mathbb{1}_{Q_{K}^{n}} \rightarrow \partial_{t} \psi, \sum_{n=1}^{N} \sum_{K \in \mathfrak{M}^{*}} \frac{\psi_{K^{*}}^{(n+1)}-\psi_{K^{*}}^{n}}{\Delta t} 1_{Q_{K^{*}}^{n}} \rightarrow \partial_{t} \psi \text { in } L^{1}(Q), \\
& \sum_{n=1}^{N} \sum_{K \in \mathfrak{M}}\left(\mathbb{P}^{\mathfrak{T}}\left(\mathbb{S}^{\Delta t}[\mathcal{S}]\right)^{n}\right)_{K} 1_{Q_{K}^{n}} \rightarrow \mathcal{S}, \sum_{n=1}^{N} \sum_{K^{*} \in \mathfrak{M}^{*}}\left(\mathbb{P}^{\mathfrak{T}}\left(\mathbb{S}^{\Delta t}[\mathcal{S}]\right)^{n}\right)_{K^{*}} 1_{Q_{K^{*}}^{n}} \rightarrow \mathcal{S} \text { in } L^{1}(Q), \\
& \sum_{n=1}^{N} \sum_{K \in \mathfrak{M}} \psi_{K}^{n} 1_{Q_{K}^{n}} \rightarrow \psi, \sum_{n=1}^{N} \sum_{K^{*} \in \mathfrak{M}^{*}} \psi_{K^{*}}^{n} \mathbb{1}_{Q_{K^{*}}^{n}} \rightarrow \psi \text { in } L^{\infty}(Q), \\
& \nabla^{\mathfrak{T}} \psi^{\overline{\mathfrak{s}}, \Delta t} \rightarrow \nabla \psi \text { in } L^{p}(Q) \text { and } \psi^{\mathfrak{m}, 1}(\cdot) \rightarrow \psi(0, \cdot), \psi^{\mathfrak{M}^{*}, 1}(\cdot) \rightarrow \psi(0, \cdot) \text { in } L^{1}(\Omega),
\end{aligned}
$$

as $h \rightarrow 0$ (here we have put Proposition 5.1 to use). Finally, the terms on the right-hand side of (60) vanish as $h \rightarrow 0$, thanks to the initial remarks made in Subsection 6.4 and Corollary 6.1(ii). In the same way, (67) follows from (61).

\section{Convergence and statement of main Result}

We are now in a position to state and prove the main result of this paper. 
Theorem 7.1. Consider a family of double meshes $\mathfrak{T}$ of $\Omega$ and associated time steps $\Delta t>0$, parametrized by $h=\max \{\operatorname{size}(\mathfrak{T}), \Delta t\}, h \rightarrow 0$. Assume that $\operatorname{reg}(\mathfrak{T})$ is uniformly bounded. Then the corresponding discrete solutions $u^{\overline{\mathfrak{s}}, \overline{\Delta t}}$ of (33), (34), (35) exist, are uniformly bounded, and converge to the unique entropy solution $u$ of (1) in the following strong sense:

$$
\begin{aligned}
& u^{\mathfrak{M}, \Delta t} \rightarrow u, u^{\mathfrak{M} *}, \Delta t \rightarrow u \text { in } L^{s}(Q) \text { for any } s<\infty, \\
& \nabla^{\mathfrak{\tau}} w^{\overline{\mathfrak{s}}, \Delta t} \rightarrow \nabla w \text { in } L^{p}(Q), \quad \text { where } w=A(u) .
\end{aligned}
$$

Proof. We follow step by step the proof of Theorem 2.1.

(i) Discrete solutions $u^{\bar{z}, \overline{\Delta t}}$ exist by Proposition 6.2 Besides, they verify the asymptotic entropy inequalities (66) (where we can replace $\eta_{c}^{ \pm}$by $\eta_{c, \varepsilon}^{ \pm}$) and the asymptotic weak formulation (67), both of Proposition 6.5.

(ii) Proposition 6.1 yields uniform estimates on both $u^{\mathfrak{M}, \Delta t}$ and $u^{\mathfrak{M}^{*}, \Delta t}$ in $L^{\infty}(Q)$; on the time translates of both $w^{\mathfrak{M}, \Delta t}$ and $w^{\mathfrak{M}^{*}, \Delta t}$ in $L^{1}(Q)$; on the penalization term $\sum_{n=1}^{N} \Delta t \llbracket\left[\mathcal{P}^{\mathfrak{T}}\left[w^{\overline{\mathfrak{s}}, n}\right], w^{\mathfrak{T}, n} \rrbracket\right.$; and on $\nabla^{\mathfrak{T}} w^{\overline{\mathfrak{s}}, \Delta t}$ in $L^{p}(Q)$. The latter estimate implies further uniform estimates: namely, an estimate of the space translates of both $w^{\mathfrak{M}, \Delta t}$ and $w^{\mathfrak{M}^{*}, \Delta t}$ in $L^{1}(Q)$, by Proposition 5.2 (ii); an estimate of $\nabla^{\mathfrak{I}} \widetilde{A}_{\left(\eta_{c, \varepsilon}^{ \pm}\right)^{\prime}}\left(w^{\overline{\mathfrak{s}}, \Delta t}\right)$ in $L^{p}(Q)$, because $\widetilde{A}_{\left(\eta_{c, \varepsilon}^{ \pm}\right)^{\prime}}(\cdot)$ is Lipschitz and by construction of $\nabla^{\mathfrak{T}}[\cdot]$; and finally an estimate of $\mathfrak{a}\left(\nabla^{\mathfrak{T}} w^{\overline{\mathfrak{s}}, \Delta t}\right)$ in $L^{p^{\prime}}(Q)$, because of the growth assumption on $\mathfrak{a}$.

(iii) Thanks to the estimates of (ii), there exists a (not labelled) sequence of $\mathfrak{T}, \Delta t$ with $h \rightarrow 0$ such that

- by the Fréchet-Kolmogorov theorem, each of the sequences $w^{\mathfrak{m}, \Delta t}$ and $w^{\mathfrak{M}^{*}, \Delta t}$ converges strongly in $L^{1}(Q)$ and pointwise a.e. in $Q$;

- by Proposition 5.3, the limits of $w^{\mathfrak{m}, \Delta t}$ and $w^{\mathfrak{M}^{*}, \Delta t}$ coincide (we denote the limit of $w^{\mathfrak{M}, \Delta t}, w^{\mathfrak{M}^{*}, \Delta t}$ by $w$ ), and $\nabla^{\mathfrak{T}} w^{\overline{\mathfrak{s}}, \Delta t}$ converges weakly in $L^{p}(Q)$ to $\nabla w$;

- $\mathfrak{a}\left(\nabla^{\mathfrak{T}} w^{\overline{\mathfrak{s}}, \Delta t}\right)$ converges weakly in $L^{p^{\prime}}(Q)$ to a limit field $\chi$;

- the sequences $u^{\mathfrak{m}, \Delta t}, u^{\mathfrak{m} *}, \Delta t$ converge to $\mu, \mu^{*}: Q \times(0,1) \rightarrow \mathbf{R}$, respectively,

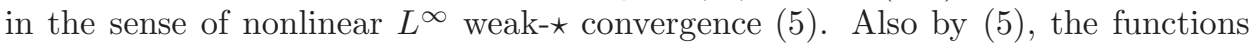
$w^{\mathfrak{m}, \Delta t}=A\left(u^{\mathfrak{m}, \Delta t}\right)$ converge to $A(\mu)$ in the $L^{\infty}$ weak- $\star$ sense; since the functions $w^{\mathfrak{m}, \Delta t}$ also converge strongly, $A(\mu)$ is independent of $\alpha$ and coincides with $w$. In the same way, we deduce that $A\left(\mu^{*}\right)$ is independent of $\alpha$ and coincides with $w$. Also observe that $u^{\mathfrak{m}, 0}, u^{\mathfrak{m} *}, 0$ both converge to $u_{0}$ a.e. in $\Omega$ and in $L^{1}(\Omega)$.

(iv) As in the proof of Theorem 2.1. we can use the chain rule and the GreenGauss formula to deduce

$$
\begin{aligned}
& \int_{Q} \int_{0}^{1} \frac{1}{d}\left(\mathfrak{f}(\mu)+(d-1) \mathfrak{f}\left(\mu^{*}\right)\right) \cdot \nabla A(u) \\
& =\frac{1}{d} \int_{0}^{1} \int_{Q}\left(\mathfrak{f}(\mu) \cdot \nabla A(\mu)+(d-1) \mathfrak{f}\left(\mu^{*}\right) \cdot \nabla A\left(\mu^{*}\right)\right)=\int_{0}^{T} \int_{\partial \Omega} \widetilde{A}_{\mathfrak{f}}(w) \cdot n=0,
\end{aligned}
$$

where $\widetilde{A}_{\mathrm{f}}$ is defined in (15).

(v) Next we pass to the limit in (67). Indeed, by (iii),

$$
\left\{\begin{array}{c}
\partial_{t} \widetilde{u}+\operatorname{div} \int_{0}^{1} \frac{1}{d}\left(\mathfrak{f}(\mu)+(d-1) \mathfrak{f}\left(\mu^{*}\right)\right) d \alpha=\operatorname{div} \chi+\mathcal{S} \\
\quad \operatorname{in} L^{p^{\prime}}\left(0, T ; W^{-1, p^{\prime}}(\Omega)\right)+L^{1}(Q),\left.\quad \widetilde{u}\right|_{t=0}=u_{0} .
\end{array}\right.
$$


where

$$
\widetilde{u}(t, x)=\int_{0}^{1} \widetilde{\mu}(t, x, \alpha) d \alpha, \quad \widetilde{\mu}=\frac{1}{d}\left(\mu+(d-1) \mu^{*}\right) .
$$

Let us identify $\chi$ (the weak limit of $\mathfrak{a}\left(\nabla^{\mathfrak{T}} w^{\overline{\mathfrak{s}}}, \Delta t\right)$ ) with $\mathfrak{a}(\nabla w)$, and consequently obtain that the weak convergence is in fact strong in $L^{p}(Q)$. To this end, we will use (iv) and (62) to establish the inequality

$$
\int_{Q} \chi \cdot \nabla w \geq \liminf _{h \rightarrow 0} \sum_{n=1}^{N} \Delta t\left\{\left\{\mathfrak{a}\left(\nabla^{\mathfrak{T}} w^{\overline{\mathfrak{s}}, n}\right), \nabla^{\mathfrak{T}} w^{\overline{\mathfrak{s}}, n}\right\} .\right.
$$

Indeed, using (69), we can represent the left-hand side of (70) as

$$
\begin{aligned}
\int_{Q} \chi \cdot \nabla w \zeta=- & \int_{0}^{T}\left\langle\partial_{t} \widetilde{u}, w \zeta\right\rangle \\
& +\int_{0}^{1} \int_{Q} \frac{1}{d}\left(\mathfrak{f}(\mu)+(d-1) \mathfrak{f}\left(\mu^{*}\right)\right) \cdot \nabla w \zeta+\int_{Q} \mathcal{S} w \zeta,
\end{aligned}
$$

where $\zeta \in \mathcal{D}([0, T))$ is nonincreasing with $\zeta(t) \equiv 1$ for $t$ small.

Note that since $A$ is nondecreasing, since $\widetilde{u}(t, x)$ is a convex combination of the values $\mu(t, x, \cdot)$ and $\mu^{*}(t, x, \cdot)$, and because $A(\mu)=A\left(\mu^{*}\right)=w$, we conclude that

$$
w=A(\widetilde{u}) .
$$

To control $\int_{0}^{T}\left\langle\partial_{t} \widetilde{u}, w \zeta\right\rangle$, we argue along the lines of the proof of Theorem 2.1. The duality product $\left\langle\partial_{t} \widetilde{u}, A(\widetilde{u})\right\rangle$ is treated via the weak chain rule (cf. [4]). Hence, exploiting also the convexity of $B(z)=\int_{0}^{z} A(s) d s$,

$$
\begin{aligned}
\int_{0}^{T}\left\langle\partial_{t} \widetilde{u}, A(\widetilde{u}) \zeta\right\rangle & \\
& =-\int_{Q} B(\widetilde{u}) \zeta^{\prime}-\int_{\Omega} B\left(u_{0}\right) \\
& =\int_{Q} B\left(\int_{0}^{1} \widetilde{\mu}(t, x, \alpha) d \alpha\right)\left(-\zeta^{\prime}\right)-\int_{\Omega} B\left(u_{0}\right) \\
& \leq-\int_{Q} \zeta^{\prime} \int_{0}^{1} B(\widetilde{\mu}(t, x, \alpha)) d \alpha-\int_{\Omega} B\left(u_{0}\right) .
\end{aligned}
$$

Using (68) and (72), we deduce from (71) that

$$
\int_{Q} \chi \cdot \nabla w \zeta \geq \int_{Q} \zeta^{\prime} \int_{0}^{1} B(\widetilde{\mu}(t, x, \alpha)) d \alpha+\int_{\Omega} B\left(u_{0}\right)+\int_{Q} \delta w .
$$

On the other hand, Proposition 6.3 permits to evaluate the right-hand side of (70) as follows:

$$
\begin{gathered}
\liminf _{h \rightarrow 0}\left(\sum _ { n = 1 } ^ { N } \Delta t \left\{\left\{\left(\mathfrak{a}\left(\nabla^{\mathfrak{T}} w^{\overline{\mathfrak{T}}, n}\right), \nabla^{\mathfrak{T}} w^{\overline{\mathfrak{T}}, n} \zeta^{\overline{\mathfrak{T}}, n}\right\}\right)\right.\right. \\
\leq \liminf _{h \rightarrow 0}\left(\sum_{n=1}^{N-1} \Delta t \llbracket B\left(u^{\mathfrak{T}, n}\right), \frac{\zeta^{\mathfrak{T},(n+1)}-\zeta^{\mathfrak{T}, n}}{\Delta t} \rrbracket+\llbracket B\left(u^{\mathfrak{T}, 0}\right), 1^{\mathfrak{T}} \rrbracket\right. \\
\left.+\sum_{n=1}^{N} \Delta t \llbracket \mathbb{P}^{\mathfrak{T}}\left(\mathbb{S}^{\Delta t}[\mathcal{S}]\right)^{n}, w^{\mathfrak{T}, n} \zeta^{\mathfrak{T}, n} \rrbracket\right)
\end{gathered}
$$


By the previously established convergences (see also the proof of Proposition 6.5), the right-hand side of (74) is equal to the right-hand side of (73). Once we let $\zeta$ tend to $\mathbb{1}_{[0, T)}$, this establishes (70).

Starting from (70), we apply the Minty-Browder argument that we employed for the continuous problem in the proof of Theorem 2.1 .

Take $v \in L^{p}\left(0, T ; W_{0}^{1, p}(\Omega)\right) \cap L^{\infty}(Q)$, and set $v^{\bar{\Sigma}}, \Delta t=\mathbb{P}^{\bar{\Sigma}} \circ \mathbb{S}^{\Delta t}[v]$. In view of (70), taking into account the strong convergence of $\nabla^{\mathfrak{s}} v^{\overline{\mathfrak{s}}, \Delta t}$ to $\nabla v$ in $L^{p}(Q)$, cf. Proposition [5.1] and the monotonicity of $\mathfrak{a}(\cdot)$ we obtain

$$
\begin{aligned}
& \int_{Q} \chi \cdot \nabla(w-v) \geq \liminf _{h \rightarrow 0} \sum_{n=1}^{N} \Delta t\left\{\left\{\mathfrak{a}\left(\nabla^{\mathfrak{\mathcal { T }}} w^{\overline{\mathfrak{s}}, n}\right), \nabla^{\mathfrak{\mathcal { T }}} w^{\overline{\mathfrak{s}}, n}-\nabla^{\mathfrak{T}} v^{\overline{\mathfrak{s}}, n}\right\}\right. \\
& \geq \liminf _{h \rightarrow 0} \sum_{n=1}^{N} \Delta t\left\{\left\{\mathfrak{a}\left(\nabla^{\mathfrak{s}} v^{\overline{\mathfrak{s}}, n}\right), \nabla^{\mathfrak{s}} w^{\overline{\mathfrak{s}}, n}-\nabla^{\mathfrak{\mathfrak { x }}} v^{\overline{\mathfrak{s}}, n}\right\}\right. \text {. }
\end{aligned}
$$

As is a well-known property of Leray-Lions operators, the strong convergence of $\nabla^{\mathfrak{s}} v^{\overline{\mathfrak{s}}}, \Delta t$ to $\nabla v$ in $L^{p}(Q)$ implies the strong convergence of $\mathfrak{a}\left(\nabla^{\mathfrak{z}} v^{\overline{\mathfrak{s}}}, \Delta t\right)$ to $\mathfrak{a}(\nabla v)$ in $L^{p^{\prime}}(Q)$. Therefore (75) yields

$$
\int_{Q} \chi \cdot \nabla(w-v) \geq \int_{Q} \mathfrak{a}(\nabla v) \cdot \nabla(w-v) .
$$

Choosing $v=w \pm \lambda \psi$ with $\lambda \downarrow 0$ and $\psi \in L^{p}\left(0, T ; W_{0}^{1, p}(\Omega)\right)$, we conclude

$$
\chi=\mathfrak{a}(\nabla w) .
$$

Moreover, as in the proof of Theorem 2.1 and [11, Theorem 5.1], relying on the strict monotonicity of $\mathfrak{a}$ and utilizing an argument of [24, 21, we also deduce the strong convergence of $\nabla^{\mathfrak{T}} w^{\overline{\mathfrak{s}}, \Delta t}$ to $\nabla w$ in $L^{p}(Q)$.

(vi) Now we can pass to the limit in the weak and entropy formulations listed in Proposition 6.5. The passage from (67) to (D'.2) is straightforward. In (66), we first work with regularized boundary entropies. Taking the limit, all the terms converge to the corresponding terms in (D'.3) in a straightforward way, except for the third one. Let us show that

$$
\nabla^{\mathfrak{s}} \widetilde{A}_{\left(\eta_{c, \varepsilon}^{ \pm}\right)^{\prime}}\left(w^{\overline{\mathfrak{s}}, \Delta t}\right) \text { converges weakly to } \nabla \widetilde{A}_{\left(\eta_{c, \varepsilon}^{ \pm}\right)^{\prime}}(w) \text { in } L^{p}(Q) \text {. }
$$

Indeed, both $\widetilde{A}_{\left(\eta_{c, \varepsilon}^{ \pm}\right)^{\prime}}\left(w^{\mathfrak{M}, \Delta t}\right)$ and $\widetilde{A}_{\left(\eta_{c, \varepsilon}^{ \pm}\right)^{\prime}}\left(w^{\mathfrak{M} *, \Delta t}\right)$ converge to $\widetilde{A}_{\left(\eta_{c, \varepsilon}^{ \pm}\right)^{\prime}}(w)$ by the a.e. convergence of $w^{\mathfrak{M}, \Delta t}, w^{\mathfrak{M} *, \Delta t}$ to $w$ and the continuity of $\widetilde{A}_{\left(\eta_{c, \varepsilon}^{ \pm}\right)^{\prime}}$. Using the boundedness in $L^{p}(Q)$ of $\left.\nabla^{\mathfrak{T}} \widetilde{A}_{\left(\eta_{c, \varepsilon}^{ \pm}\right)^{\prime}}\left(w^{\bar{s}, \Delta t}\right)\right)$ and the compactness property of Proposition 5.3. we conclude that our claim holds. The subsequent arguments are the same as in the proof of Theorem 2.1.

(vii) We conclude that $\left(\mu, \mu^{*}, w\right)$ is an entropy double-process solution of (1). In view of Theorem 2.2. this brings to an end the proof of Theorem 7.1, indeed, we obtain the convergence to $u$ for each sequence of discrete solutions with $h \rightarrow 0$. Also, the fact that $\mu$ and $\mu^{*}$ turn out to be independent of $\alpha$ means that the convergence of $u^{\mathfrak{m}, \Delta t}, u^{\mathfrak{m}, \Delta t}$ to $u$ is strong in $L^{s}(Q)$ for all finite $s$.

\section{ON THE CHOICE OF FV SCHEME AND VARIOUS GENERALIZATIONS}

In this section we discuss other possible choices of finite volume schemes for (1). 
- The use of DDFV schemes is motivated by their convenience when it comes to the discretization of nonlinear diffusion operators. Other possibilities exist; among them, let us mention the schemes studied in [56. (see also [3, 12, 8]), in [10, in [42] (see also [47), and in [52] (see also [50, 51, 53]). All these schemes possess some variant of the "integration-by-parts" property of Proposition 4.1

The 2D schemes of 10 are restricted to Cartesian meshes, so they do not allow for domains much more general than rectangles. Notice that their generalization to 3D appears to be straightforward. The techniques used in the present paper and in the references we cite, such as [48, 49, combined with those of 10, allow to design rather simple FV schemes on Cartesian meshes for problem (11) and to prove their convergence. In this case, the notion of entropy double-process solution is not needed, and the theoretical results in [49] can be adapted directly.

This is also the case of the "complementary volumes" schemes as described in [56]. In 2D, ideas quite similar to that of [56] were used to construct the schemes of [3, 12, 8]. All these schemes work on meshes dual to conformal triangular 2D meshes, and the discrete gradient is reconstituted by affine per triangle interpolation. "Complementary volumes" schemes are simpler than our DDFV scheme from the practical point of view, since one discretizes the problem on the same mesh $\mathfrak{M}^{*}$ using, roughly speaking, half of the unknowns. The discrete duality properties for the 2D "complementary volumes" scheme are shown in the same way as for our DDFV schemes; the proof is based upon Lemma 9.6 (see Appendix B and also [7, 8]). Unfortunately, the straightforward generalization of these "complementary volumes" schemes to 3D fails to satisfy the discrete duality property, except for very constrained geometries of the meshes (see Remark 9.2).

The key feature of the 2D schemes of [3, 56, 12, 10, 8, (see also 40]) lies in the fact that the fluxes across interfaces are reconstructed "manually". The approaches of Droniou and Eymard [47, 42] and those of the HVF, SUCCES and SUSHI schemes of Eymard, Gallouët and Herbin 50, 51, 53, 52 are different; they rely on introducing additional unknowns (either for the fluxes, or for the values on some of the edges) and on careful penalization of the finite differences.

The schemes HVF, SUCCES and SUSHI (among many others) were designed for handling linear anisotropic, heterogeneous diffusion problems with possibly discontinuous coefficients; in this framework, their convergence is justified. These schemes avoid usage of double meshes and thus may have less unknowns; they work both in 2D and 3D. We refer to Eymard, Gallouët and Herbin [52] for the description and comparison of these and related (e.g., mimetic finite difference) schemes. Finally, let us also mention the schemes of Aavatsmark et al. (see, e.g., [1, 2]), that are in a sense intermediate. The gradient reconstruction used in [1, 2] also involves additional edge unknowns, which are eliminated by solving, locally, an algebraic system of equations.

The scheme of 42 designed for nonlinear Leray-Lions kind problems can be directly compared to the DDFV schemes of 11] and of the present paper. The scheme of 42 is very interesting because of the extreme generality of the geometries allowed for the mesh (and it works in any space dimension). For this same reason, theoretical justification of its convergence in the hyperbolic-parabolic framework (11) seems problematic. Indeed, the conformity (orthogonality) condition was used in an essential way in the derivation of the discrete entropy inequalities (see Remark 
4.1). The same difficulty arises for the double schemes of [11] in the case of nonconformal meshes, cf. Remark 3.2. In passing, let us point out that the conformity (orthogonality) assumption on the meshes is the only condition that is known to ensure the discrete maximum principle for the DDFV schemes.

In conclusion, the 2D and 3D conformal DDFV schemes studied herein, although constrained by the orthogonality condition, by the Delaunay condition, and by condition (19), combine some degree of flexibility (e.g., any polygonal/polyhedral domain can be partitioned into triangles/tetrahedra satisfying these restrictions) with the rigid structure properties underlying our convergence proof. But because of the conformity constraint, the advantage of simple local refinement procedures for 2D DDFV schemes, pointed out in [11, is lost.

- Our assumption that $\mathfrak{M}$ consists of simplexes is a practical one simplifying the presentation of the scheme. In $2 \mathrm{D}$, it can be replaced by the more general assumption that any element of $\mathfrak{M}$ admits a circumscribed circle. In $3 \mathrm{D}$, we can assume that each $K \in \overline{\mathfrak{M}}$ admits a circumscribed ball, and each interface $K \mid L$ is a triangle satisfying (19).

Notice that Remark 9.3 (see also [7) makes it possible to define a consistent discrete duality scheme even when the interfaces $K \mid L$ are not necessarily triangles. Unfortunately, the discrete Poincaré inequality may fail in this generality; this undermines the subsequent convergence analysis. Yet one interesting case is that of a Cartesian mesh $\mathfrak{M}$; the corresponding DDFV schemes are alternatives to the scheme of [10] discussed above. More generally, one can start with a mesh $\mathfrak{M}$ made of rectangles (e.g., inside $\Omega$ ) and triangles (e.g., near the boundary $\partial \Omega$ ) in $2 \mathrm{D}$.

- As pointed out in Remark 3.5, a different kind of reconstruction formula is needed for problems in $4 \mathrm{D}$ and higher dimensions. It would be interesting to conceive discrete gradients consistent with affine functions, following the principle formulated in Remark 3.3. One natural way is indicated in 38.

- The choice of penalization in our double scheme can be changed (see Remark (3.6). One could also penalize the differences $\left(u_{K}-u_{K^{*}}\right)$ instead of the differences $\left(w_{K}-w_{K^{*}}\right)=\left(A\left(u_{K}\right)-A\left(u_{K^{*}}\right)\right)$; this would permit to avoid the use of doubleprocess solutions. But this choice would introduce additional coupling between the sets of variables $\left(u_{K}\right)_{K \in \mathfrak{M}}$ and $\left(u_{K^{*}}\right)_{K^{*} \in \mathfrak{M}^{*}}$ in the "hyperbolic" regions. Indeed, if, e.g., $A(u) \equiv 0$, there is no coupling at all between the variables sitting on $\mathfrak{M}$ and those sitting on $\mathfrak{M}^{*}$. Therefore our choice seems more convenient in terms of practical implementation.

- Convection-diffusion problems with anisotropic linear and nonlinear diffusion were considered in [35, 34] and in [17, 18. General DDFV schemes do not seem easy to adapt to the nonlinear anisotropic framework, because of the presence of "privileged" directions of diffusion. In this case, the schemes of [10] on Cartesian meshes constitute a natural choice, and the geometry of $\partial \Omega$ should be rather taken into account via the approximation of the domain $\Omega$ by domains with piecewise axes-aligned boundaries. Notice that for the anisotropic $p$-Laplace kind diffusions

$$
\partial_{x_{1}}\left(\left|\partial_{x_{1}} A_{1}(u)\right|^{p_{1}-2} \partial_{x_{1}} A_{1}(u)\right)+\partial_{x_{2}}\left(\left|\partial_{x_{2}} A_{2}(u)\right|^{p_{2}-2} \partial_{x_{2}} A_{2}(u)\right)
$$

considered by Bendahmane, Karlsen in [17, 18, the discrete entropy inequalities on Cartesian meshes are as easy to obtain as for the isotropic case $\mathfrak{a}(\xi)=k(\xi) \xi$ considered in the present paper.

- Taking into account sufficiently smooth dependencies on $(t, x)$ of the convection and diffusion operators is possible, although quite technical; see [48, 11] for some 
results in that direction, and also [33, 60, for well-posedness results for degenerate equations with $(t, x)$ dependent convection-diffusion operators. Discontinuous coefficients are important for the modeling of fractured media. DDFV schemes for Leray-Lions operators $\operatorname{div} \mathfrak{a}(x, \nabla w)$ with discontinuous (piecewise smooth) in $x$ nonlinearity $\mathfrak{a}$ are studied in the recent work 23 . The case of $x$-discontinuous flux functions $\mathfrak{f}(x, u)$ has received much attention in the last fifteen years (see, e.g., [26]

and the references cited therein), both from a theoretical and numerical perspective. Let us mention here that the problem of the choice of the appropriate entropy conditions strongly depends on the underlying physical interpretation; different models lead to qualitatively different admissible solutions.

- Inhomogeneous Dirichlet boundary conditions can be taken into account, combining the techniques of [67] with those of [11; both are rather involved, which explains our choice of the homogeneous boundary data for the presentation of the scheme and the convergence arguments.

\section{Appendix A: Proof of uniqueness}

This appendix is devoted to a proof of Theorem 2.2. The proof is an adaptation of the ones in Carrillo 29. (for entropy solutions) and that in Eymard, Gallouët, Herbin, Michel 49] (for entropy process solutions, which can be viewed as entropy double-process solutions with $\left.\mu \equiv \mu^{*}\right)$. The proof is mainly divided into several lemmas (Lemmas 9.1 9.3, 9.5 below). For simplicity, let us only consider the case where the source term $\mathcal{S}$ is zero (see also Remark 9.1).

We begin by introducing the set

$$
\mathcal{E}=\left\{r \in \mathbf{R}: A^{-1}(r) \text { is neither empty nor a singleton }\right\},
$$

and proving

Lemma 9.1. Let $\left(\nu, \nu^{\star}, v\right)$ be an entropy double-process solution of (11) with initial data $v_{0}$. Then for all $W \in \mathbf{R}^{d}$, for any $\phi \in \mathcal{D}([0, T) \times \Omega), c \in \mathbf{R}$ such that $A(c) \notin \mathcal{E}$ and also for any $\phi \in \mathcal{D}([0, T) \times \bar{\Omega}), c \in \mathbf{R}^{ \pm}$such that $A(c) \notin \mathcal{E}$, we have with the notation of Section 2 the following equality:

$$
\begin{aligned}
& \int_{0}^{1} \int_{Q} {\left[\frac{1}{d}\left(\eta_{c}^{ \pm}(\nu)+(d-1) \eta_{c}^{ \pm}(\nu)\right) \partial_{t} \phi+\frac{1}{d}\left(\mathfrak{q}_{c}^{ \pm}(\nu)+(d-1) \mathfrak{q}_{c}^{ \pm}\left(\nu^{\star}\right)\right) \cdot \nabla \phi\right.} \\
&\left.\left.\quad-\operatorname{sign}^{ \pm}(v-A(c))(\mathfrak{a}(\nabla v)-W)\right) \cdot \nabla \phi\right] d x d t d \alpha+\left.\int_{\Omega} \eta_{c}^{ \pm}\left(v_{0}\right) \phi\right|_{t=0} d x \\
&=\lim _{\varepsilon \downarrow 0} \int_{Q}\left(\operatorname{sign}_{\varepsilon}^{ \pm}\right)^{\prime}(v-A(c))(\mathfrak{a}(\nabla v)-W) \cdot \nabla v \phi d x d t .
\end{aligned}
$$

Proof. We refer to [29, Lemma 1] and to [49] for details on the proof. The idea is to use $\psi:=\left(\operatorname{sign}_{\varepsilon}^{ \pm}(v-A(c)) \phi\right)$ as a test function in (D'.2). It is admissible; indeed, we can approximate it by functions in $\mathcal{D}([0, T) \times \Omega)$ and pass to the limit in all terms of (D'.2), because $\psi \in L^{\infty}(Q) \cap L^{p}\left(0, T ; W_{0}^{1, p}(\Omega)\right)$ for any of the two possible choices of $(\phi, c)$ (in particular, notice that $\operatorname{sign}_{\varepsilon}^{ \pm}(v-A(c)) \in L^{p}\left(0, T ; W_{0}^{1, p}(\Omega)\right.$ ) in case $c \in \mathbf{R}^{ \pm}$). We have

$$
\operatorname{sign}^{ \pm}(\nu-c)=\operatorname{sign}^{ \pm}(v-A(c))=\operatorname{sign}^{ \pm}\left(\nu^{*}-c\right),
$$

thanks to the relation (D'.1) (which reads $A(\nu) \equiv v \equiv A\left(\nu^{*}\right)$ in our notation) and to the choice of $A(c) \notin \mathcal{E}$; then we use the weak chain rule to deal with the time 
derivative. We also insert into (D'.2) the term

$$
\int_{0}^{1} \int_{Q} \operatorname{sign}_{\varepsilon}^{ \pm}(v-A(c)) \nabla \phi \cdot W-\int_{0}^{1} \int_{Q}\left(\operatorname{sign}_{\varepsilon}^{ \pm}\right)^{\prime}(v-A(c)) \nabla w \cdot W \phi,
$$

which is equal to $0=\int_{Q} \operatorname{div}\left(W \operatorname{sign}_{\varepsilon}^{ \pm}(v-A(c)) \phi\right)$ for any of the two possible choices of $(\phi, c)$, by the Gauss-Green formula. As $\varepsilon \downarrow 0$, the term containing $\left(\operatorname{sign}_{\varepsilon}^{ \pm}\right)^{\prime}(v-A(c))(\mathfrak{f}(\nu)-\mathfrak{f}(c)) \cdot \nabla v$ vanishes, as shown in [29, Lemma 1].

We are now interested in comparing two entropy double-process solutions of (11), denoted by $\left(\nu, \nu^{*}, \theta\right)$ and $\left.\left(\mu, \mu^{*}, w\right)\right)$, of which the first one is chosen to satisfy $\nu \equiv \nu^{*}$. Consider the distribution $\mathcal{I}$ on $\mathcal{D}(\bar{Q})$ defined by

$$
\begin{aligned}
& \mathcal{I}[\phi]:=\int_{0}^{1} \int_{0}^{1} \int_{Q}\left[\frac{1}{d}\left((\nu-\mu)^{+}+(d-1)\left(\nu-\mu^{\star}\right)^{+}\right) \partial_{t} \phi\right. \\
&+ \frac{1}{d}\left(\operatorname{sign}^{+}(\nu-\mu)(\mathfrak{f}(\nu)-\mathfrak{f}(\mu))\right. \\
&+\left.(d-1) \operatorname{sign}^{+}\left(\nu-\mu^{\star}\right)\left(\mathfrak{f}(\nu)-\mathfrak{f}\left(\mu^{\star}\right)\right)\right) \cdot \nabla \phi \\
&\left.-\operatorname{sign}^{+}(v-w)(\mathfrak{a}(\nabla v)-\mathfrak{a}(\nabla w)) \cdot \nabla \phi\right] d x d t d \alpha d \beta \\
&+\int_{\Omega}\left(v_{0}-u_{0}\right)^{+} \phi(0, x) d x .
\end{aligned}
$$

Let us prove that we can write $\mathcal{I}$ as

$$
\mathcal{I}=\mathcal{I P}+\mathcal{I N}
$$

where $\mathcal{I P}[\phi]$ is defined by the analogue of (77) with each of $\nu, v, v_{0}, \mu, \mu^{*}, w, u_{0}$ replaced by its positive part; and $\mathcal{I N}[\phi]$ is defined by the analogue of (77) with each of $\nu, v, v_{0}, \mu, \mu^{*}, w, u_{0}$ replaced by $-\nu^{-},-v^{-},-v_{0}^{-},-\mu^{-},-\left(\mu^{*}\right)^{-},-w^{-},-u_{0}^{-}$. To emphasize, whenever necessary, the dependency of $\mathcal{I}, \mathcal{I} \mathcal{P}, \mathcal{I N}$ on the involved solutions, we will write $\mathcal{I}_{\mu, \mu^{*}, w}^{\nu, \nu,}, \mathcal{I P}_{\mu, \mu^{*}, w}^{\nu, \nu, v}, \mathcal{I N}_{\mu, \mu^{*}, w}^{\nu, \nu, v}$, respectively.

To justify (78), we use the identity (79) from the following easy lemma.

Lemma 9.2. For all $F: \mathbf{R} \rightarrow \mathbf{R}$ such that $F(0)=0$, for all $a, b \in \mathbf{R}$ there holds

$$
\begin{aligned}
\operatorname{sign}^{+}(a-b)(F(a)-F(b))= & \operatorname{sign}^{+}\left(a^{+}-b^{+}\right)\left(F\left(a^{+}\right)-F\left(b^{+}\right)\right) \\
& +\operatorname{sign}^{+}\left(\left(-a^{-}\right)-\left(-b^{-}\right)\right)\left(F\left(-a^{-}\right)-F\left(-b^{-}\right)\right),
\end{aligned}
$$

and

$$
\begin{aligned}
& \text { (i) } \operatorname{sign}^{-}\left(b-a^{+}\right) F(b)=-\operatorname{sign}^{+}\left(a^{+}-b^{+}\right) F\left(b^{+}\right)+\operatorname{sign}^{-}(b) F(b), \\
& \text { (ii) } \operatorname{sign}^{-}\left(b-a^{+}\right) F\left(a^{+}\right)=-\operatorname{sign}^{+}\left(a^{+}-b^{+}\right) F\left(a^{+}\right) .
\end{aligned}
$$

We apply (79) to $a=\nu, b=\mu\left(\right.$ or $\left.b=\mu^{*}\right)$ with $F=\operatorname{Id}$ and with $F=\mathfrak{f}_{i}, i=$ $1, \ldots, d$. Futhermore, observe that the analogue of (79) still holds for a.e. $(t, x) \in Q$ if we take $a=v(t, x), b=w(t, x)$ and replace $F(a), F(b)$ and $F\left( \pm a^{ \pm}\right), F\left( \pm b^{ \pm}\right)$ by $\mathfrak{a}(\nabla v), \mathfrak{a}(\nabla w)$ and by $\mathfrak{a}\left( \pm \nabla v^{ \pm}\right), \mathfrak{a}\left( \pm \nabla w^{ \pm}\right)$, respectively. Indeed, we have, e.g., $\mathfrak{a}(\nabla v)=\mathfrak{a}\left(\nabla v^{+}\right)+\mathfrak{a}\left(\nabla v^{-}\right)$a.e. on $Q$, because $v \in L^{p}\left(0, T ; W_{0}^{1, p}(\Omega)\right)$. Using all aforementioned identities, we split each term in the definition (77) of $\mathcal{I}$ into the sum of the corresponding terms in the definitions of $\mathcal{I P}$ and $\mathcal{I N}$.

Now we estimate $\mathcal{I}$ "inside" the domain". 
Lemma 9.3. Let $(\nu, \nu, v)$ and $\left(\mu, \mu^{\star}, w\right)$ be entropy double-process solutions of (1) with data $v_{0}, u_{0} \in L^{\infty}(\Omega)$, respectively. Then $\mathcal{I}[\phi] \geq 0, \forall \phi \in \mathcal{D}([0, T) \times \Omega), \phi \geq 0$.

Proof. The proof is an application of the doubling of variables method of Kruzhkov 63]; it follows [29, 49, 17]. We let $\nu$ depend on variables $(t, x, \alpha) \in Q \times(0,1)$ and $\mu$ depend on another set of variables $(s, y, \beta) \in Q \times(0,1)$. In what follows, $\nabla v$ means $\nabla_{x} v$ and $\nabla w$ means $\nabla_{y} w$. As to the test function $\phi$, it will depend on the variables $(t, s, x, y)$, thus we will use the notations $\partial_{t}, \partial_{s}$ and $\nabla_{x}, \nabla_{y}$ for the corresponding derivatives of $\phi$. We will work with nonnegative test functions $\phi \in \mathcal{D}\left(([0, T) \times \Omega)^{2}\right)$. Let us introduce the sets on which the diffusion term for the first, respectively, for the second solution degenerates:

$$
\mathcal{E}_{\nu}=\{(t, x) \in Q \mid v(t, x) \in \mathcal{E}\}, \quad \mathcal{E}_{\mu}=\{(s, y) \in Q \mid w(s, y) \in \mathcal{E}\} .
$$

Denote by $\mathcal{E}_{\nu}^{c}, \mathcal{E}_{\mu}^{c}$ the complementary sets in $Q$ of $\mathcal{E}_{\nu}, \mathcal{E}_{\mu}$, respectively. Observe that $\nabla v=0$ a.e. in $\mathcal{E}_{\nu}$ and $\nabla w=0$ a.e. in $\mathcal{E}_{\mu}$ (recall (D'.1)].

(i) First we apply Lemma 9.1 with the solution $(\nu, \nu, v)$. For all $(s, y, \beta) \in$ $\mathcal{E}_{\mu} \times(0,1)$, choose $W=\mathfrak{a}(\nabla w(s, y))$ and take the entropy $\eta_{c}^{+}(\cdot)=(\cdot-c)^{+}$with $c=\mu(s, y, \beta)$, then with $c=\mu^{\star}(s, y, \beta)$ in (76). We multiply the two resulting equations by $\frac{1}{d}$ and by $\frac{(d-1)}{d}$, respectively, and add them together. Then we integrate in $(s, y, \beta) \in \mathcal{E}_{\mu} \times(0,1)$. Similarly, for $(s, y, \beta) \in \mathcal{E}_{\mu}^{c} \times(0,1)$, we add together, with weights $\frac{1}{d}$ and $\frac{(d-1)}{d}$, respectively, the entropy inequalities (D'.3) for $(\nu, \nu, v)$ corresponding to $\eta_{c}^{+}(\cdot)$ with $c=\mu(s, y, \beta)$ and with $c=\mu^{\star}(s, y, \beta)$. We integrate the resulting inequality in $(s, y, \beta) \in \mathcal{E}_{\mu}^{c} \times(0,1)$.

(ii) Next, we exchange the roles of $(\nu, \nu, v)$ and $\left(\mu, \mu^{*}, w\right)$. This time we use the entropy $\eta_{c}^{-}(\cdot)=(\cdot-c)^{-}$; we use $W=\mathfrak{a}(\nabla v(t, x))$; and we only use one value $c=\nu(t, x, \alpha)$ in the analogue of (76) (for all $\left.(t, x, \alpha) \in \mathcal{E}_{\nu} \times(0,1)\right)$ and in the analogue of (D'.3) (for all $(t, x, \alpha) \in \mathcal{E}_{\nu}^{c} \times(0,1)$ ).

(iii) Adding the inequalities obtained in (i),(ii), by the symmetry of the expressions involved (such as $(\nu-\mu)^{+}=(\mu-\nu)^{-}$, etc.), we get, keeping in mind Remark 2.1. the following inequality:

$$
\begin{gathered}
\int_{0}^{1} \int_{0}^{1} \iint_{Q \times Q}\left[\frac{1}{d}\left((\nu-\mu)^{+}+(d-1)\left(\nu-\mu^{\star}\right)^{+}\right)\left(\partial_{t}+\partial_{s}\right) \phi\right. \\
+\frac{1}{d}\left(\operatorname{sign}^{+}(\nu-\mu)(\mathfrak{f}(\nu)-\mathfrak{f}(\mu))\right. \\
\left.\quad+(d-1) \operatorname{sign}^{+}\left(\nu-\mu^{\star}\right)\left(\mathfrak{f}(\nu)-\mathfrak{f}\left(\mu^{\star}\right)\right)\right)\left(\nabla_{x}+\nabla_{y}\right) \phi \\
\left.\quad-\operatorname{sign}^{+}(v-w)(\mathfrak{a}(\nabla v)-\mathfrak{a}(\nabla w)) \cdot\left(\nabla_{x}+\nabla_{y}\right) \phi\right] d x d t d y d s d \alpha d \beta \\
+\int_{0}^{1} \iint_{\Omega \times Q} \frac{1}{d}\left(\left(v_{0}-\mu\right)^{+}+(d-1)\left(v_{0}-\mu^{\star}\right)^{+}\right) \phi d x(d y d s) d \beta \\
+\int_{0}^{1} \iint_{Q \times \Omega}\left(\nu-u_{0}\right)^{+} \phi(d x d t) d y d \alpha \\
\geq \lim _{\varepsilon \downarrow 0} \iint_{\mathcal{E}_{\nu}^{c} \times \mathcal{E}_{\mu}^{c}}\left(\operatorname{sign}_{\varepsilon}^{+}\right)^{\prime}(v-w)(\mathfrak{a}(\nabla v)-\mathfrak{a}(\nabla w)) \cdot(\nabla v-\nabla w) \phi d x d t d y d s .
\end{gathered}
$$

The last term in (81) is nonnegative, because $\mathfrak{a}$ is monotone and $\phi \geq 0$.

(iv) Let us now specify the test function. For $l, n \in \mathbb{N}$, let $\omega_{n}: \mathbf{R}^{d} \rightarrow \mathbf{R}$, $\omega^{l}: \mathbf{R} \rightarrow \mathbf{R}$ be standard symmetric mollifiers with supports in $\left\{x \in \mathbf{R}^{d} \mid\|x\| \leq \frac{1}{n}\right\}$ 
and in $\left\{t \in \mathbf{R}|| t \mid \leq \frac{1}{l}\right\}$, respectively. We take the test function in (81) to be

$$
\phi_{n, l}(t, x, s, y)=\phi(x, t) \omega_{n}(x-y) \omega^{l}(t-s) \equiv \phi \omega_{n} \omega^{l},
$$

where $\phi \in \mathcal{D}([0, T) \times \Omega), \phi \geq 0$. With this choice, we have

$$
\left(\partial_{t}+\partial_{s}\right) \phi_{n, l}=\left(\partial_{t} \phi\right) \omega_{n} \omega^{l}, \quad\left(\nabla_{x}+\nabla_{y}\right) \phi_{n, l}=\left(\nabla_{x} \phi\right) \omega_{n} \omega^{l} .
$$

Then we let $n, l \rightarrow \infty$. The first term in (81) converges to the first term in the right-hand side of (77). This argument is standard; one can use, e.g., the properties of the Lebesgue points of $L^{1}$ functions and the upper-semicontinuity of the $L^{1}$ "+bracket"

$$
[u, f]_{+}:=\int_{\Omega} \operatorname{sign}^{+}(u) f+\int_{\{u=0\}} f^{+} .
$$

The two latter terms in the left-hand side of (810) are treated with the help of the triangular inequality and of the strong initial trace property (83) proved in Lemma 9.4 below. The limit, as $n, l \rightarrow \infty$, of each of these terms is majorated by one half of the last term in (777) (this is because $\int_{Q} \omega^{l}(t) d t=\frac{1}{2}=\int_{Q} \omega^{l}(-s) d s$ ). This concludes the proof of the lemma.

Lemma 9.4. Let $\left(\mu, \mu^{\star}, w\right)$ be an entropy double-process solution of (11) with initial datum $u_{0} \in L^{\infty}(\Omega)$. Then the initial datum is also taken in the following strong sense:

$$
\lim _{h \downarrow 0} \frac{1}{h} \int_{0}^{h} \int_{\Omega} \int_{0}^{1}\left(\frac{1}{d}\left|\mu-u_{0}\right|+\frac{d-1}{d}\left|\mu^{\star}-u_{0}\right|\right) d t d x d \alpha=0 .
$$

Notice that another way to formulate (83) is to say that

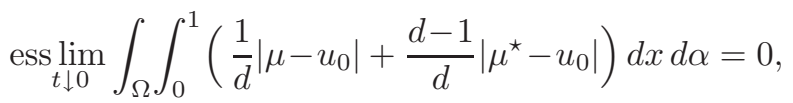

in the spirit of the original definition of Kruzhkov 63.

Proof. The proof follows the one of Panov in [71, Proposition 1]. For $c \in \mathbf{R}$ and $h>0$, consider the functions

$$
p_{h}(\cdot ; c): x \in \Omega \mapsto \frac{1}{h} \int_{0}^{h} \int_{0}^{1}\left(\frac{1}{d}|\mu(t, x ; \alpha)-c|+\frac{d-1}{d}\left|\mu^{\star}(t, x ; \alpha)-c\right|\right) d t d \alpha .
$$

Because $\mu, \mu^{*}$ are bounded, the set $\left(p_{h}(\cdot ; c)\right)_{h>0}$ is bounded in $L^{\infty}(\Omega)$. Therefore for any sequence $h_{n} \rightarrow 0$, there exists a subsequence (not relabeled) such that for all $c \in \mathbb{Q},\left(p_{h}(\cdot ; c)\right)_{h>0}$ converges in $L^{\infty}(\Omega)$ weak- $\star$ to some limit denoted by $p(\cdot ; c)$.

Fix $\xi \in \mathcal{D}(\Omega), \xi \geq 0$. From Definition 2.3. taking in (D'.2) test functions approaching $\psi(t, x):=\left(1-\frac{t}{h_{n}}\right)^{+} \xi(x)$ we readily infer the inequalities

$$
\forall c \in \mathbb{Q} \quad \int_{\Omega} p(x ; c) \xi(x) d x \leq \int_{\Omega}\left|u_{0}(x)-c\right| \xi(x) d x .
$$

By the density argument, we extend (84) to all $\xi \in L^{1}(\Omega), \xi \geq 0$.

Now for all $\delta>0$, there exists a number $N(\delta) \in \mathbb{N}$, a collection $\left(c_{i}^{\delta}\right)_{i=1}^{N(\delta)} \subset \mathbb{Q}$ and a partition of $\Omega$ into disjoint union of measurable sets $\Omega_{1}^{\delta}, \ldots, \Omega_{N(\delta)}^{\delta}$ such that $\left\|u_{0}-u_{0}^{\delta}\right\|_{L^{1}} \leq \delta$, where

$$
u_{0}^{\delta}:=\sum_{i=1}^{N(\delta)} c_{i}^{\delta} 1_{\Omega_{i}^{\delta}} .
$$


Because $\mathbb{1}_{\Omega}=\sum_{i=1}^{N(\delta)} 1_{\Omega_{i}^{\delta}}$, applying (84) with $c=c_{i}^{\delta}$ and $\xi=1_{\Omega_{i}^{\delta}}$ we deduce

$$
\begin{aligned}
\lim _{n \rightarrow \infty} & \frac{1}{h_{n}} \int_{0}^{h_{n}} \int_{\Omega} \int_{0}^{1}\left(\frac{1}{d}\left|\mu-u_{0}^{\delta}\right|+\frac{d-1}{d}\left|\mu^{\star}-u_{0}^{\delta}\right|\right) d t d x d \alpha \\
\quad & \lim _{n \rightarrow \infty} \int_{\Omega} \sum_{i=1}^{N(\delta)} p_{h_{n}}\left(x ; c_{i}^{\delta}\right) 1_{\Omega_{i}^{\delta}}(x) d x=\int_{\Omega} \sum_{i=1}^{N(\delta)} p\left(x ; c_{i}^{\delta}\right) 1_{\Omega_{i}^{\delta}}(x) d x \\
& \leq \int_{\Omega} \sum_{i=1}^{N(\delta)}\left|u_{0}(x)-c_{i}^{\delta}\right| 1_{\Omega_{i}^{\delta}}(x) d x=\left\|u_{0}-u_{0}^{\delta}\right\|_{L^{1}} \leq \delta .
\end{aligned}
$$

Using once more the bound $\left\|u_{0}-u_{0}^{\delta}\right\|_{L^{1}} \leq \delta$ (in the first term of the previous calculation), we can send $\delta$ to zero and infer the analogue of (83), with a limit taken on some subsequence of $\left(h_{n}\right)_{n>1}$. Because $\left(h_{n}\right)_{n>1}$ was an arbitrary sequence convergent to zero, (83) is justified.

Lemma 9.3 tells us that $\mathcal{I}[\cdot]$, which is a distribution on $[0, T) \times \bar{\Omega}$, is nonnegative when restricted to $\mathcal{D}([0, T) \times \Omega)$ and thus it is a locally finite measure on $[0, T) \times \Omega$.

Now we show that $\mathcal{I}[\phi]$ is nonnegative also for nonnegative test functions $\phi$ that do not necessarily vanish on the boundary $[0, T) \times \partial \Omega$.

Lemma 9.5. Let $(\nu, \nu, v),\left(\mu, \mu^{\star}, w\right)$ be entropy double-process solutions of (11) with initial data $v_{0}, u_{0} \in L^{\infty}(\Omega)$, respectively. Then $\mathcal{I}[\phi] \geq 0, \forall \phi \in \mathcal{D}([0, T) \times \bar{\Omega}), \phi \geq 0$.

Proof. We begin by modifying steps (i)-(iv) of the proof of the previous lemma; we refer to this proof for the notation and a part of the calculations.

(i) We use (766) and (D'.3) in the same way as in the proof of Lemma 9.3 but we choose the values $c=\mu^{+}(s, y, \beta), c=\left(\mu^{\star}\right)^{+}(s, y, \beta)$ and $W=\mathfrak{a}\left(\nabla w^{+}(s, y)\right)$ instead of the values $c=\mu(s, y, \beta), c=\mu^{\star}(s, y, \beta)$ and $W=\mathfrak{a}(\nabla w(s, y))$, respectively.

Notice that for all $a, b \in \mathbf{R}, \varepsilon \geq 0$, we have $\operatorname{sign}_{\varepsilon}^{+}\left(a-b^{+}\right)=\operatorname{sign}_{\varepsilon}^{+}\left(a^{+}-b^{+}\right)$and, moreover, this expression is zero whenever $a \leq 0$. Thus we can replace $\nu, v, \nabla v$ by $\nu^{+}, v^{+}, \nabla v^{+}$everywhere in this calculation and obtain

$$
\begin{gathered}
\int_{0}^{1} \int_{0}^{1} \iint_{Q \times Q}\left[\frac{1}{d}\left(\left(\nu^{+}-\mu^{+}\right)^{+}+(d-1)\left(\nu^{+}-\left(\mu^{\star}\right)^{+}\right)^{+}\right) \partial_{t} \phi\right. \\
+\frac{1}{d}\left(\operatorname{sign}^{+}\left(\nu^{+}-\mu^{+}\right)\left(\mathfrak{f}\left(\nu^{+}\right)-\mathfrak{f}\left(\mu^{+}\right)\right)\right. \\
\left.+(d-1) \operatorname{sign}^{+}\left(\nu^{+}-\left(\mu^{\star}\right)^{+}\right)\left(\mathfrak{f}\left(\nu^{+}\right)-\mathfrak{f}\left(\left(\mu^{\star}\right)^{+}\right)\right)\right) \cdot \nabla_{x} \phi
\end{gathered}
$$

(ii) We follow the proof of Lemma9.3 but choose $c=\nu^{+}(t, x, \alpha), W=\mathfrak{a}\left(\nabla v^{+}(t, x)\right)$ instead of $c=\nu(t, x, \alpha), W=\mathfrak{a}(\nabla w(t, x))$.

Let us apply identities (80) to $a=\nu, b=\mu$ (or $b=\mu^{*}$ ) with $F=\mathrm{Id}$ and with $F=\mathfrak{f}_{i}, i=1, \ldots, d$. Moreover, as in the proof of (78), we also have the analogue of (80) $(i)$ with $a=v, b=w$ with $F(w)$ replaced by $\mathfrak{a}(\nabla w)$. In the same way, we also have the analogue of (80) $(i i)$ with $a=v, b=w$, and $F(v)$ replaced by $\mathfrak{a}(\nabla v)$. 
Furthermore, $\operatorname{sign}^{ \pm}(\cdot)$ can be replaced by $\left(\operatorname{sign}_{\varepsilon}^{ \pm}\right)^{\prime}(\cdot)$ in the above properties. In conclusion, we obtain

$$
\begin{gathered}
\int_{0}^{1} \int_{0}^{1} \iint_{Q \times Q}\left[\frac{1}{d}\left(\left(\nu^{+}-\mu^{+}\right)^{+}+(d-1)\left(\nu^{+}-\left(\mu^{\star}\right)^{+}\right)^{+}\right) \partial_{s} \phi\right. \\
+\frac{1}{d}\left(\operatorname{sign}^{+}\left(\nu^{+}-\mu^{+}\right)\left(\mathfrak{f}\left(\nu^{+}\right)-\mathfrak{f}\left(\mu^{+}\right)\right)\right. \\
\left.\quad+(d-1) \operatorname{sign}^{+}\left(\nu^{+}-\left(\mu^{\star}\right)^{+}\right)\left(\mathfrak{f}\left(\nu^{+}\right)-\mathfrak{f}\left(\left(\mu^{\star}\right)^{+}\right)\right)\right) \cdot \nabla_{y} \phi \\
\left.\quad-\operatorname{sign}^{+}\left(v^{+}-w^{+}\right)\left(\mathfrak{a}\left(\nabla v^{+}\right)-\mathfrak{a}\left(\nabla w^{+}\right)\right) \cdot \nabla_{y} \phi\right] d x d t d y d s d \alpha d \beta \\
+\int_{0}^{1} \int_{Q \times \Omega}\left(\nu^{+}-u_{0}^{+}\right)^{+} \phi(d x d t) d y d \alpha \\
\geq \lim _{\varepsilon \downarrow 0} \iint_{\mathcal{E}_{\nu}^{c} \times \mathcal{E}_{\mu}^{c}}\left(\operatorname{sign}_{\varepsilon}^{+}\right)^{\prime}\left(v^{+}-w^{+}\right)\left(\mathfrak{a}\left(\nabla w^{+}\right)-\mathfrak{a}\left(\nabla v^{+}\right)\right) \cdot \nabla w^{+} \phi d x d t d y d s \\
+\lim _{\varepsilon \downarrow 0} \iint_{\mathcal{E}_{\nu}^{c} \times \mathcal{E}_{\mu}^{c}}\left(\operatorname{sign}_{\varepsilon}^{+}\right)^{\prime}(-w) \mathfrak{a}(\nabla w) \cdot \nabla w \phi d x d t d y d s \\
-\int_{0}^{1} \int_{0}^{1} \iint_{Q \times Q} \frac{1}{d}\left[\operatorname{sign}^{-}(\mu)\left\{\mu \partial_{s} \phi+(\mathfrak{f}(\mu)-\mathfrak{a}(\nabla w)) \cdot \nabla_{y} \phi\right\}\right. \\
\left.\quad+(d-1) \operatorname{sign}{ }^{-}\left(\mu^{*}\right)\left\{\mu^{*} \partial_{s} \phi+\left(\mathfrak{f}\left(\mu^{*}\right)-\mathfrak{a}(\nabla w)\right) \cdot \nabla_{y} \phi\right\}\right] d x d t d y d s d \alpha d \beta \\
-\int_{Q \times \Omega}\left(u_{0}\right)^{-} \phi(d x d t) d y .
\end{gathered}
$$

Notice that the sum of the last two terms in (86) can be rewritten under the form $-\mathcal{L}_{\mu, \mu^{*}}(\chi)$, where

$$
\chi(s, y)=\int_{Q} \phi(t, s, x, y) d t d x \in \mathcal{D}([0, T) \times \bar{\Omega})
$$

and the distribution $\mathcal{L}_{\mu, \mu^{*}}$ is defined on $\mathcal{D}([0, T) \times \bar{\Omega})$ by

$$
\begin{aligned}
\mathcal{L}_{\mu, \mu^{*}}(\chi):= & \int_{0}^{1} \int_{Q}\left[\frac{1}{d}\left(\eta_{0}^{-}(\mu)+(d-1) \eta_{0}^{-}\left(\mu^{*}\right)\right) \partial_{s} \chi\right. \\
& +\frac{1}{d}\left(\mathfrak{q}_{0}^{-}(\mu)+(d-1) \mathfrak{q}_{0}^{-}\left(\mu^{*}\right)\right) \cdot \nabla_{y} \chi \\
& \left.-k(\nabla w) \nabla \widetilde{A}_{\left(\eta_{0}^{-}\right)^{\prime}}(w) \cdot \nabla_{y} \chi\right] d y d s d \beta+\int_{\Omega} \eta_{0}^{-}\left(u_{0}\right) \chi d y .
\end{aligned}
$$


(iii) Adding (85) and (86), we obtain, for any $0 \leq \phi \in \mathcal{D}\left(([0, T) \times \bar{\Omega})^{2}\right)$ with corresponding $\chi$ defined in (87), the following inequality:

$$
\begin{aligned}
& \int_{0}^{1} \int_{0}^{1} \iint_{Q \times Q}\left[\frac{1}{d}\left(\left(\nu^{+}-\mu^{+}\right)^{+}+(d-1)\left(\nu^{+}-\left(\mu^{\star}\right)^{+}\right)^{+}\right)\left(\partial_{t}+\partial_{s}\right) \phi\right. \\
& +\frac{1}{d}\left(\operatorname{sign}^{+}\left(\nu^{+}-\mu^{+}\right)\left(\mathfrak{f}\left(\nu^{+}\right)-\mathfrak{f}\left(\mu^{+}\right)\right)\right. \\
& \left.\quad+(d-1) \operatorname{sign}^{+}\left(\nu^{+}-\left(\mu^{\star}\right)^{+}\right)\left(\mathfrak{f}\left(\nu^{+}\right)-\mathfrak{f}\left(\left(\mu^{\star}\right)^{+}\right)\right)\right) \cdot\left(\nabla_{x}+\nabla_{y}\right) \phi \\
& \left.\quad-\operatorname{sign}^{+}\left(v^{+}-w^{+}\right)\left(\mathfrak{a}\left(\nabla v^{+}\right)-\mathfrak{a}\left(\nabla w^{+}\right)\right) \cdot\left(\nabla_{x}+\nabla_{y}\right) \phi\right] d x d t d y d s d \alpha d \beta \\
& +\int_{0}^{1} \iint_{\Omega \times Q} \frac{1}{d}\left(\left(v_{0}^{+}-\mu^{+}\right)^{+}+(d-1)\left(v_{0}^{+}-\left(\mu^{\star}\right)^{+}\right)^{+}\right) \phi d x(d y d s) d \beta \\
& +\int_{0}^{1} \iint_{Q \times \Omega}\left(\nu^{+}-u_{0}^{+}\right)^{+} \phi(d x d t) d y d \alpha \\
& \geq \lim _{\varepsilon \downarrow 0} \iint_{\mathcal{E}_{\nu}^{c} \times \mathcal{E}_{\mu}^{c}}\left(\operatorname{sign}_{\varepsilon}^{+}\right)^{\prime}\left(v^{+}-w^{+}\right)\left(\mathfrak{a}\left(\nabla v^{+}\right)-\mathfrak{a}\left(\nabla w^{+}\right)\right) \cdot\left(\nabla v^{+}-\nabla w^{+}\right) \phi d x d t d y d s \\
& +\lim _{\varepsilon \downarrow 0} \iint_{\mathcal{E}_{\nu}^{c} \times \mathcal{E}_{\mu}^{c}}\left(\operatorname{sign}_{\varepsilon}^{+}\right)^{\prime}(-w) \mathfrak{a}(\nabla w) \cdot \nabla w \phi d x d t d y d s-\mathcal{L}_{\mu, \mu^{*}}(\chi) \geq-\mathcal{L}_{\mu, \mu^{*}}(\chi),
\end{aligned}
$$

where the last inequality is due to the monotonicity of $\mathfrak{a}(\cdot)$.

(iv) Now fix $x_{0} \in \partial \Omega$. Since $\partial \Omega$ is supposed sufficiently regular, there exists a vector $r_{x_{0}}$ and a positive number $R_{x_{0}}$ such that the segment $\left(x, x+r_{x_{0}}\right]$ lies within $\Omega$ for all $x \in \partial \Omega \cap B\left(x_{0}, R_{x_{0}}\right)$, where $B(x, R)$ stands for the ball of $\mathbf{R}^{d}$ with centre $x$ and radius $R$. Choose in (89) the sequence of test functions

$$
\phi_{n, l}(t, x, s, y)=\phi(y, s) \omega_{n}\left(x-y+\frac{2}{n} \frac{r_{x_{0}}}{\left\|r_{x_{0}}\right\|}\right) \omega_{l}(t-s) \equiv \phi \omega_{n} \omega^{l},
$$

for which (82) still holds. Notice that with this choice, the associated function $\chi_{n, l}(y, s)$ in (87) writes as $\phi(y, s) \theta_{n}(y) \theta^{l}(s)$, where

$$
\theta_{n}(y):=\int_{\Omega} \omega_{n}\left(x-y+\frac{2}{n} \frac{r_{x_{0}}}{\left\|r_{x_{0}}\right\|}\right) d x, \quad \theta^{l}(s):=\int_{0}^{T} \omega_{l}(t-s) d t
$$

moreover, for all sufficiently large $n \in \mathbb{N}$ we have

$$
\mid \begin{aligned}
& \phi \theta_{n} \in \mathcal{D}(\Omega) \text { for all } \phi \in \mathcal{D}\left([0, T) \times\left(\bar{\Omega} \cap B\left(x_{0}, R_{x_{0}}\right)\right)\right) ; \\
& \theta_{n}(y)=1 \text { for all } y \in B\left(x_{0}, R_{x_{0}}\right) \text { such that dist }(y, \partial \Omega) \geq \frac{3}{n} .
\end{aligned}
$$

As in the proof of Lemma 9.3. passing to the limit as $l, n \rightarrow \infty$ and taking into account the definition of the distribution $\mathcal{I P}$, cf (78), from (89) we deduce

$$
\mathcal{I P}[\phi] \geq-\liminf _{l, n \rightarrow \infty} \mathcal{L}_{\mu, \mu^{*}}\left[\phi \theta_{n} \theta_{l}\right] .
$$

Now we remark that according to (D'.3) $\mathcal{L}_{\mu, \mu^{*}}$ defined by (88) is a nonnegative distribution on $[0, T) \times \bar{\Omega}$. Notice that the values of $\theta_{n}$ are contained in the interval $[0,1]$. Therefore for all $\chi \in \mathcal{D}([0, T) \times \bar{\Omega}), \phi \geq 0$, one has

$$
\mathcal{L}_{\mu, \mu^{*}}\left[\phi \theta_{n}\right]=\mathcal{L}_{\mu, \mu^{*}}[\phi]-\mathcal{L}_{\mu, \mu^{*}}\left[\phi\left(1-\theta_{n}\right)\right] \leq \mathcal{L}_{\mu, \mu^{*}}[\phi] .
$$

It follows that

$$
\overline{\mathcal{L}}_{\mu, \mu^{*}}: \chi \in \mathcal{D}([0, T) \times \bar{\Omega}) \mapsto \liminf _{n \rightarrow \infty} \mathcal{L}_{\mu, \mu^{*}}\left(\chi \theta_{n}\right)
$$


is a nonnegative distribution on $[0, T) \times \bar{\Omega}$; thus, it is a measure on $[0, T) \times \bar{\Omega}$. Since $\phi \geq 0$ and $\theta^{l} \leq 1$, inequality (91) yields

$$
\mathcal{I P}[\phi] \geq-\liminf _{l, n \rightarrow \infty} \mathcal{L}_{\mu, \mu^{*}}\left[\chi \theta_{n} \theta_{l}\right] \geq-\liminf _{n \rightarrow \infty} \mathcal{L}_{\mu, \mu^{*}}\left[\phi \theta_{n}\right]=-\overline{\mathcal{L}}_{\mu, \mu^{*}}[\phi] .
$$

It follows that $\mathcal{I P}$ is a measure on $[0, T) \times \bar{\Omega}$.

The remaining steps of the proof are aimed at showing, in an indirect way, that the positive part of the measure $\mathcal{I P}$ does not charge the boundary $[0, T) \times \partial \Omega$ (in two particular cases, a direct proof of this fact is given in [73, 13]). Notice that this property is actually equivalent to the claim of the lemma; it accounts for the dissipative nature of the boundary condition imposed for entropy solutions.

(v) Take $\phi \in \mathcal{D}\left([0, T) \times\left(\bar{\Omega} \cap B\left(x_{0}, R_{x_{0}}\right)\right)\right)$. Fix $m \in \mathbb{N}$. It is easily checked from (90) that for all sufficiently large $n \in \mathbb{N}$, for all $(t, x) \in Q$,

$$
\phi(s, y)\left(1-\theta_{m}(y)\right) \theta_{n}(y)=\phi(s, y) \theta_{n}(y)-\phi(s, y) \theta_{m}(y) .
$$

Therefore by (92),

$$
\begin{aligned}
& \liminf _{m \rightarrow \infty} \overline{\mathcal{L}}_{\mu, \mu^{*}}\left[\phi\left(1-\theta_{m}\right)\right] \\
& \quad=\liminf _{m \rightarrow \infty} \liminf _{n \rightarrow \infty} \mathcal{L}_{\mu, \mu^{*}}\left[\phi \theta_{n}\right]-\liminf _{m \rightarrow \infty} \liminf _{n \rightarrow \infty} \mathcal{L}_{\mu, \mu^{*}}\left[\phi \theta_{m}\right]=0 .
\end{aligned}
$$

Applying (93) to the test function $\phi\left(1-\theta_{m}\right)$, we deduce

$$
\begin{aligned}
\mathcal{I P}_{\mu, \mu^{*}, w}^{\nu, \nu, v}[\phi] & =\mathcal{I P}_{\mu, \mu^{*}, w}^{\nu, \nu}\left[\phi \theta_{m}\right]+\mathcal{I P}_{\mu, \mu^{*}, w}^{\nu, \nu, v}\left[\phi\left(1-\theta_{m}\right)\right] \\
& \geq \limsup _{m \rightarrow \infty} \mathcal{I P}_{\mu, \mu^{*}, w}^{\nu, \nu, v}\left[\phi \theta_{m}\right] .
\end{aligned}
$$

(vi) Definition 2.3 of entropy double-process solution is invariant under the change of $\left(\mu, \mu^{*}, w\right), \mathfrak{f}, f, u_{0}$ into $\left(-\mu,-\mu^{*},-w\right),-\mathfrak{f},-f,-u_{0}$. Moreover, one checks easily from the definition, cf. (78), that $\mathcal{I N}_{\mu, \mu^{*}, w}^{\nu, \nu, w}=\mathcal{I P}_{-\nu,-\nu,-v}^{-\mu,-\mu^{*},-w}$. Therefore from (94) we deduce that for all $\phi \in \mathcal{D}\left([0, T) \times\left(\bar{\Omega} \cap B\left(x_{0}, R_{x_{0}}\right)\right)\right)$

$$
\begin{aligned}
& \mathcal{I}_{\mu, \mu^{*}, w}^{\nu, \nu, v}[\phi]=\mathcal{I P}_{\mu, \mu^{*}, w}^{\nu, \nu, v}[\phi]+\mathcal{I N}_{\mu, \mu^{*}, w}^{\nu, \nu, v}[\phi]=\mathcal{I P}_{\mu, \mu^{*}, w}^{\nu, \nu, v}[\phi]+\mathcal{I P}_{-\nu,-\nu,-v}^{-\mu,-\mu^{*},-w}[\phi] \\
& \geq \limsup _{m \rightarrow \infty}\left[\mathcal{I P}_{\mu, \mu^{*}, w}^{\nu, \nu, v}\left[\phi \theta_{m}\right]+\mathcal{I P}_{-\nu,-\nu,-v}^{-\mu,-\mu^{*},-w}\left[\phi \theta_{m}\right]\right]=\limsup _{m \rightarrow \infty} \mathcal{I}_{\mu, \mu^{*}, w}^{\nu, \nu, v}\left[\phi \theta_{m}\right] \geq 0,
\end{aligned}
$$

where the last inequality is due to (90) and Lemma 9.3

(vii) Not let $\phi$ be an arbitrary nonnegative function in $\mathcal{D}([0, T) \times \bar{\Omega})$. Choose a covering $\bigcup_{i=1}^{N} B\left(x_{0}^{i}, R_{x_{0}^{i}}\right), N \in \mathbb{N}$, of the compact set $\partial \Omega$. Introduce a partition of unity $\left(\xi_{i}\right)_{i=0}^{N}$ on $\bar{\Omega}$ associated with the covering $\Omega \bigcup\left(\bigcup_{i=1}^{N} B\left(x_{0}^{i}, R_{x_{0}^{i}}\right)\right)$ of $\bar{\Omega}$, and apply Lemma 9.3 and the result of (vi) to the functions $\phi \xi_{0} \in \mathcal{D}([0, T) \times \Omega)$ and to $\phi \xi_{i} \in \mathcal{D}\left([0, T) \times\left(\bar{\Omega} \cap B\left(x_{0}^{i}, R_{x_{0}^{i}}\right)\right)\right), i=1, \ldots, N$, respectively. The claim of the lemma follows.

Now we conclude the proof of Theorem 2.2 We have $u_{0}=v_{0}$. By a standard argument, choosing in Lemma $9.5 \phi=\phi(t) \in \mathcal{D}([0, T))$, we get for a.e. $t \in(0, T)$,

$$
\int_{0}^{1} \int_{0}^{1} \int_{\Omega} \frac{1}{d}\left((\nu(t, x, \alpha)-\mu(t, x, \beta))^{+}+(d-1)\left(\nu(t, x, \alpha)-\mu^{\star}(t, x, \beta)\right)^{+}\right) \leq 0 .
$$

Now, (95) means that for a.e. $(x, \alpha, \beta) \in \Omega \times(0,1) \times(0,1)$, there holds

$$
\mu(t, x, \beta)=\nu(t, x, \alpha)=\mu^{*}(t, x, \beta),
$$


which means that $\mu \equiv \mu^{*} \equiv \nu$ and each of them is independent of $\alpha, \beta$. This draws to a close the proof of Theorem 2.2

Remark 9.1. The proof of the $L^{1}$ contraction and comparison principle for entropy solutions of (11) (with $\mathcal{S}=0$ ) is essentially contained in the above proof. For nonzero source terms $\mathcal{S}$, a more general version of inequalities (80) can be used; see [29] for the accurate treatment of this term.

\section{Appendix B: The ReCOnstruction PROperty}

Here we restate the result of [12, Lemma 8] and discuss its possible generalizations.

Lemma 9.6. Consider a triangle $\mathbb{T}$ with vertices $t_{1}, t_{2}, t_{3}$ and let $t_{0}$ be the centre of its circumscribed circle. Denote by $|\mathbb{T}|$ its area. For $l \in \mathbb{N} / 3 \mathbb{N}$, denote by $E_{l}$ the affine subspace $\left\langle\overrightarrow{t_{l-1} t_{l+1}}\right\rangle$; denote by $\mathbb{T}_{l}$ the triangle formed by $t_{0}, t_{l-1}, t_{l+1}$ and by $\left|\mathbb{T}_{l}\right|$ its area, with the convention that the area is negative if $t_{0}$ and $t_{l}$ lay on opposite sides from the line passing by $t_{l-1}, t_{l+1}$. Then

$$
\frac{2}{|\mathbb{T}|} \sum_{l=1}^{3}\left|\mathbb{T}_{l}\right| \operatorname{Proj}_{E_{l}}(\vec{r})=\vec{r}, \quad \text { for all } \vec{r} \in \mathbf{R}^{2} .
$$

Remark 9.2. For a multi-D generalization of the property (96), one could try to replace the projections on lines $\left\langle E_{l}\right\rangle$ by projections on hyperplanes that contain the faces of the $d$-dimensional simplex $\mathbb{T}$. In this case one should replace the factor $\frac{2}{|T|}$ by $\frac{d}{d-1} \frac{1}{|T|}$, since $|T|=\sum_{l=1}^{d+1}\left|\mathbb{T}_{l}\right|$ and because the dimension of $\operatorname{Proj}_{E_{l}}(\vec{r})$ is $(d-1)$, whereas the dimension of $\vec{r}$ is $d$. The proof of Lemma 9.6 given below shows that this generalization fails, except for very particular simplexes $\mathbb{T}$ (this is clear from the multi-dimensional analogue of the identity (97) below).

Remark 9.3. Using the "sine theorem", another proof of Lemma 9.6 can be given, which also works for any $2 \mathrm{D}$ polygon that admits a circumscribed circle.

Proof. Proof of Lemma 9.6 For $l \in \mathbb{N} / 3 \mathbb{N}$, denote by $d_{l}$ the orthogonal projection if the point $t_{l}$ on the affine subspace $E_{l}$; set $\vec{p}_{l}=\overrightarrow{t_{0} d_{l}}$ and $\vec{a}_{l}={\overrightarrow{t_{0}}}_{l}$. For $l, i \in \mathbb{N} / 3 \mathbb{N}$, set $\vec{b}_{l, i}=\vec{a}_{i}-\vec{a}_{l}$. Denote by $\vec{n}_{l}$ the exterior to $\mathbb{T}$ unit normal vector to $E_{l}$. Notice that we have for all $l \in \mathbb{N} / 3 \mathbb{N}, \vec{d}_{l}=\left(\vec{d}_{l} \cdot \vec{n}_{l}\right) \vec{n}_{l}$, and also, for all $i \in \mathbb{N} / 3 \mathbb{N}$ such that $i \neq l$,

$$
\frac{\left|T_{l}\right|}{|\mathbb{T}|}=\frac{\vec{p}_{l} \cdot \vec{n}_{l}}{\vec{b}_{l, i} \cdot \vec{n}_{l}}
$$

taking into account the sign of $\left|\mathbb{T}_{l}\right|$. Since $\operatorname{Proj}_{E_{l}}+\operatorname{Proj}_{<\vec{n}_{l}>}$ is the identity operator, (96) is equivalent to the statement that $\frac{2}{|T|} \sum_{l=1}^{3}\left|\mathbb{T}_{l}\right| \operatorname{Proj}_{<\vec{n}_{l}>}$ is the identity operator. All vector $\vec{r} \in \mathbf{R}^{2}$ can be uniquely represented under the form

$$
\vec{r}=\sum_{l=1}^{3} k_{l} \vec{a}_{l} \quad \text { with } \quad \sum_{l=1}^{3} k_{l}=0,
$$


and thus, for all $\left.l \in \mathbb{N} / 3 \mathbb{N}, \vec{r}=\sum_{i \neq l, i=1}^{3} k_{i} \overrightarrow{ }_{(} a_{i}-\vec{a}_{l}\right)=\sum_{i \neq l, i=1}^{3} k_{i} \vec{b}_{l, i}$. Hence

$$
\begin{aligned}
& \frac{2}{|\mathbb{T}|} \sum_{l=1}^{3}\left|\mathbb{T}_{l}\right| \operatorname{Proj}_{<\vec{n}_{l}>}(\vec{r})=2 \sum_{l=1}^{3} \frac{\left|\mathbb{T}_{l}\right|}{|T|} \vec{n}_{l}\left(\vec{r} \cdot \vec{n}_{l}\right)=2 \sum_{i \neq l ; i, l=1}^{3} \vec{n}_{l} \frac{\left|\mathbb{T}_{l}\right|}{|\mathbb{T}|} k_{i}\left(\vec{b}_{l, i} \cdot \vec{n}_{l}\right) \\
& =2 \sum_{l=1}^{3} \vec{n}_{l} \sum_{i \neq l, i=1}^{3} \frac{\vec{p}_{l} \cdot \vec{n}_{l}}{\vec{b}_{l, i} \cdot \vec{n}_{l}} k_{i}\left(\vec{b}_{l, i} \cdot \vec{n}_{l}\right)=2 \sum_{l=1}^{3} \vec{p}_{l} \sum_{i \neq l, i=1}^{3} k_{i}=-2 \sum_{l=1}^{3} k_{l} \vec{p}_{l} .
\end{aligned}
$$

We conclude that (96) is equivalent to the identity

$$
\sum_{l=1}^{3} k_{l} \vec{a}_{l}=-2 \sum_{l=1}^{3} k_{l} \vec{p}_{l} \quad \text { for all } k_{1}, \ldots, k_{3} \in \mathbf{R} \text { such that } \sum_{l=1}^{3} k_{l}=0 .
$$

Since $t_{0}$ is the centre of the circumscribed circle of $\mathbb{T}$, the points $d_{l}$ are the centres of the corresponding segments $\left[t_{i-1}, t_{i+1}\right]$. Thus for all $i, j \in \mathbb{N} / 3 \mathbb{N}$, by the Thales theorem we have $\vec{p}_{i}-\vec{p}_{j}=-\frac{1}{2}\left(\vec{a}_{i}-\vec{a}_{j}\right)$. Hence (97) holds with $k_{i} \in\{0,1,-1\}$, $i=1, \ldots, 3$. Hence it holds for all choice of $k_{i}$.

We refer to [7, 8] for a different kind of generalization of [12, Lemma 8] and a different proof of Lemma 9.6.

\section{REFERENCES}

[1] I. Aavatsmark. An introduction to multipoint flux approximations for quadrilateral grids. Locally conservative numerical methods for flow in porous media., Comput. Geosci. 6(3-4): 405-432, 2002. 18

[2] I. Aavatsmark, T. Barkve, Ø. Bœ, and T. Mannseth. Discretization on unstructured grids for inhomogeneous, anisotropic media. Part I: derivation of the methods. SIAM J.Sci.Comp. 19(5):1700-1716, 1998. 1 8

[3] M. Afif and B. Amaziane. Convergence of finite volume schemes for a degenerate convectiondiffusion equation arising in flows in porous media, Comput. Methods Appl. Mech. Eng. 191(46):5265-5286, 2002. 10

[4] H. W. Alt and S. Luckhaus. Quasilinear elliptic-parabolic differential equations. Math. Z. 183(3):311-341, 1983. 12 220

[5] K. Ammar, P. Wittbold and J. Carrillo. Scalar conservation laws with general boundary condition and continuous flux function. J. Diff. Eq. 228(1):111-139, 2006. 11 1

[6] K. Ammar and P. Wittbold. Existence of renormalized solutions of degenerate ellipticparabolic problems. Proc. Roy. Soc. Edinburgh Sect. A 133(3):477-496, 2003. 1

[7] B. Andreianov and M. Bendahmane. On Discrete Duality Finite Volume discretization of gradient and divergence operators in $3 \mathrm{D}$, preprint 13.13 .23 .33 .38

[8] B. Andreianov, M. Bendahmane and K.H. Karlsen. A gradient reconstruction formula for finite volume schemes and discrete duality, in R. Eymard and J. M. Herard, eds., Proceedings of Finite Volumes for Complex Applications V, Hermès, 2008. $103.23 .3 \quad 3.380$

[9] B. Andreianov, M. Bendahmane, K.H. Karlsen and S. Ouaro. Well-posedness results for triply nonlinear degenerate parabolic equations, preprint, http://www.math.ntnu.no/conservation/2008/031.html 2

[10] B. Andreianov, F. Boyer and F. Hubert. Finite volume schemes for the $p$-Laplacian on Cartesian meshes. M2AN Math. Model. Numer. Anal. 38(6):931-960, 2005. 18

[11] B. Andreianov, F. Boyer and F. Hubert. Discrete duality finite volume schemes for LerayLions type elliptic problems on general 2D meshes. Num. Meth. PDE 23(1):145-195, 2007.

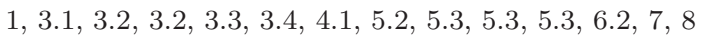

[12] B. Andreianov, M. Gutnic and P. Wittbold. Convergence of finite volume approximations for a nonlinear elliptic-parabolic problem: a "continuous" approach. SIAM J. Numer. Anal. 42(1):228-251, 2004 15.3 88

[13] B. Andreianov and N. Igbida. Uniqueness for inhomogeneous Dirichlet problem for ellipticparabolic equations. Proc. Royal Soc. Edinburgh A 137(6):1119-1133, 2007. 18 
[14] D. Aregba-Driollet, R. Natalini and S. Tang. Explicit diffusive kinetic schemes for nonlinear degenerate parabolic systems. Math. Comp. 73(245):63-94, 2004. 1

[15] C. Bardos, A. Y. LeRoux and J.-C. Nédélec. First order quasilinear equations with boundary conditions. Comm. Partial Differential Equations 4(9):1017-1034, 1979. 1

[16] J.W. Barrett and W.B. Liu. Finite element approximation of the parabolic $p$-Laplacian. SIAM J. Numer. Anal. 31(2):413-428, 1994. 1

[17] M. Bendahmane and K. H. Karlsen. Renormalized entropy solutions for quasilinear anisotropic degenerate parabolic equations. SIAM J. Math. Anal. 36(2):405-422, 2004. 1 8

[18] M. Bendahmane and K. H. Karlsen. Uniqueness of entropy solutions for doubly nonlinear anisotropic degenerate parabolic equations. Contemporary Mathematics 371, Amer. Math. Soc., pp.1-27, 2005. 10 8

[19] P. Bénilan and H. Touré. Sur l'équation générale $u_{t}=a\left(\cdot, u, \phi(\cdot, u)_{x}\right)_{x}+v$ dans $L^{1}$. II. Le problème d'évolution. Ann. Inst. H. Poincaré Anal. Non Linéaire 12(6):727-761, 1995. 1

[20] P. Bénilan and P. Wittbold. On mild and weak solutions of elliptic-parabolic problems. Adv. Differential Equations 1(6):1053-1073, 1996. 1

[21] L. Boccardo, F. Murat and J.-P. Puel. Existence of bounded solutions for nonlinear elliptic unilateral problems, Ann. Math. Pura Appl. 152(1):183-196, 1988. 20 7

[22] F. Bouchut, F. R. Guarguaglini and R. Natalini. Diffusive BGK approximations for nonlinear multidimensional parabolic equations. Indiana Univ. Math. J. 49(2):723-749, 2000. 1

[23] F. Boyer and F. Hubert. Finite volume method for 2D linear and nonlinear elliptic problems with discontinuities. SIAM J. Num. Anal. 46(6):3032-3070, 2008. 18

[24] F. Browder. Existence theorems for nonlinear partial differential equations, in Proc. Symp. Pure Math., S.S. Chern and S. Smale, eds., AMS, Providence, Rhode Island, 1970, pp.1-60. 2017

[25] R. Bürger, S. Evje and K. H. Karlsen. On strongly degenerate convection-diffusion problems modeling sedimentation-consolidation processes. J. Math. Anal. Appl. 247(2):517-556, 2000. 1

[26] R. Bürger, K. H. Karlsen, S. Mishra and J. Towers. On conservation laws with discontinuous flux. In: Y. Wang and K. Hutter (Eds.), Trends in Applications of Mathematics to Mechanics, Shaker Verlag, Aachen, pp.75-84, 2005. 8

[27] M. C. Bustos, F. Concha, R. Bürger and E. M. Tory. Sedimentation and Thickening: Phenomenological Foundation and Mathematical Theory. Kluwer Academic Publishers, Dordrecht, The Netherlands, 1999. 1

[28] J. Carrillo. On the uniqueness of the solution of the evolution dam problem. Nonlinear Anal. 22(5):573-607, 1994. 1

[29] J. Carrillo. Entropy solutions for nonlinear degenerate problems. Arch. Rational Mech. Anal.

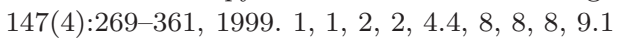

[30] J. Carrillo and P. Wittbold. Uniqueness of renormalized solutions of degenerate ellipticparabolic problems. J. Differential Equations 156(1):93-121, 1999. 102

[31] C. Chainais-Hillairet. Finite volume schemes for a nonlinear hyperbolic equation. Convergence towards the entropy solution and error estimate. M2AN Math. Model. Numer. Anal. 33(1):129-156, 1999. 12

[32] G.-Q. Chen and E. DiBenedetto. Stability of entropy solutions to the Cauchy problem for a class of nonlinear hyperbolic-parabolic equations. SIAM J. Math. Anal. 33(4):751-762, 2001. 1

[33] G.-Q. Chen and K. H. Karlsen. Quasilinear anisotropic degenerate parabolic equations with time-space dependent diffusion coefficients. Commun. Pure Appl. Anal. 4(2):241-266, 2005. 8

[34] G.-Q. Chen and K. H. Karlsen. $L^{1}$ framework for continuous dependence and error estimates for quasi-linear degenerate parabolic equations. Trans. Amer. Math. Soc. 358(3):937-963, 2006. 18

[35] G.-Q. Chen and B. Perthame. Well-posedness for non-isotropic degenerate hyperbolicparabolic equations. Ann. Inst. H. Poincaré Anal. Non Linéaire 20(4):645-668, 2003. [1 8

[36] S.-S. Chow. Finite element error estimates for non-linear elliptic equations of monotone type. Numer. Math. 54(4):373-393, 1989. 1 
[37] B. Cockburn and G. Gripenberg. Continuous dependence on the nonlinearities of solutions of degenerate parabolic equations. J. Differential Equations 151(2):231-251, 1999. 1

[38] Y. Coudiere and F. Hubert. General 3D discrete duality FV meshes. in preparation 13.28

[39] Y. Coudiere and Ch. Pierre. Stability and convergence of a finite volume method for two systems of reaction-diffusion equations in electro-cardiology. Nonlinear Anal. Real World Appl. 7(4):916-935, 2006 1 3.2

[40] Y. Coudière, J.-P. Vila and Ph. Villedieu. Convergence rate of a finite volume scheme for a two-dimensional convection-diffusion problem. M2AN Math. Model. Numer. Anal. 33(3):493516, 1999. 8

[41] K. Domelevo and P. Omnès. A finite volume method for the Laplace equation on almost arbitrary two-dimensional grids. M2AN Math. Model. Numer. Anal. 39(6):1203-1249, 2005. (document) 13.13 .24 .1

[42] J. Droniou. Finite volume approximations for fully nonlinear elliptic equations in divergence form. M2AN Math. Model. Numer. Anal. 40(6):1069-1100, 2006. 1 28

[43] M. S. Espedal and K. H. Karlsen. Numerical solution of reservoir flow models based on large time step operator splitting algorithms. In Filtration in Porous Media and Industrial Applications (Cetraro, Italy, 1998), volume 1734 of Lecture Notes in Mathematics, pp:9-77. Springer, Berlin, 2000. 1

[44] S. Evje and K. H. Karlsen. Discrete approximations of $B V$ solutions to doubly nonlinear degenerate parabolic equations. Numer. Math. 86(3):377-417, 2000. 1

[45] S. Evje and K. H. Karlsen. Monotone difference approximations of $B V$ solutions to degenerate convection-diffusion equations. SIAM J. Numer. Anal. 37(6):1838-1860, 2000. 1

[46] S. Evje, K. H. Karlsen and N. H. Risebro. A continuous dependence result for nonlinear degenerate parabolic equations with spatially dependent flux function. In Hyperbolic problems: theory, numerics, applications, Vol. I (Magdeburg, 2000), pp. 337-346. Birkhäuser, Basel, 2001. 1

[47] R. Eymard and J. Droniou. A mixed finite volume scheme for anisotropic diffusion problems on any grid. Numer. Math. 105(1):35-71, 2006. 8

[48] R. Eymard, T. Gallouët and R. Herbin. Finite Volume Methods. Handbook of Numerical Analysis, Vol. VII, P. Ciarlet, J.-L. Lions, eds., North-Holland, 2000. 12 23.1 3.23 .53 .5 3.5 $4.4 \quad 6.26 .4 \quad 6.46 .18$

[49] R. Eymard, T. Gallouët, R. Herbin and A. Michel. Convergence of a finite volume scheme for nonlinear degenerate parabolic equations. Numer. Math. 92(1):41-82, 2002. 1 126.2 $8 8 \longdiv { 8 }$

[50] R. Eymard, T. Gallouët and R. Herbin. A cell-centered finite-volume approximation for anisotropic diffusion operators on unstructured meshes in any space dimension. IMA J. Numer. Anal. 26(2):326-353, 2006. 18

[51] R. Eymard, T. Gallouët and R. Herbin. A new finite volume scheme for anisotropic diffusion problems on general grids: convergence analysis. C. R. Math. Acad. Sci. Paris 344(6):403406, 2006. 10

[52] R. Eymard, T. Gallouët and R. Herbin. Discretisation of heterogeneous and anisotropic diffusion problems on general non-conforming meshes. SUSHI: a scheme using stabilisation and hybrid interfaces, preprint HAL, http://hal.archives-ouvertes.fr/hal-00203269 18

[53] R. Eymard and R. Herbin. A new collocated FV scheme for the incompressible Navier-Stokes equations on general non-matching grids. C. R. Math. Acad. Sci. Paris 344(10):659-662, 2007. 18

[54] T. Gallouët and F. Hubert. On the convergence of the parabolic approximation of a conservation law in several space dimensions. no. 1, 141. Chinese Ann. Math. Ser. B 20(1):7-10, 1999. 2

[55] F. Guarguaglini, V. Milisic and A. Terracina. A discrete BGK approximation for strongly degenerate parabolic problems with boundary conditions. J. Diff. Eq. 202(2):183-207, 2004. 1

[56] A. Handlovičová, K. Mikula and F. Sgallari. Semi-implicit complementary volume scheme for solving level set like equations in image processing and curve evolution, Numer. Math. 93(4):675-695, 2003. 8

[57] F. Hermeline. A finite volume method for the approximation of diffusion operators on distorted meshes. J. Comput. Phys. 160(2):481-499, 2000. 1 3.13 .2 
[58] F. Hermeline. Approximation of 2D and 3D diffusion operators with discontinuous full-tensor coefficients on arbitrary meshes. Comput. Meth. Appl. Mech. Engrg. 196:2497-2526, 2007. 1 3.2

[59] N. Igbida and J. M. Urbano. Uniqueness for nonlinear degenerate problems. NoDEA Nonlinear Differential Equations Appl. 10(3):287-307, 2003. 1

[60] K. H. Karlsen and M. Ohlberger. A note on the uniqueness of entropy solutions of nonlinear degenerate parabolic equations. J. Math. Anal. Appl. 275(1):439-458, 2002. 18

[61] K. H. Karlsen and N. H. Risebro. Convergence of finite difference schemes for viscous and inviscid conservation laws with rough coefficients. M2AN Math. Model. Numer. Anal. 35(2):239-269, 2001. 1

[62] K. H. Karlsen and N. H. Risebro. On the uniqueness and stability of entropy solutions of nonlinear degenerate parabolic equations with rough coefficients. Discrete Contin. Dyn. Syst. 9(5):1081-1104, 2003. 1

[63] S. N. Kružkov. First order quasi-linear equations in several independent variables. Math. USSR Sbornik 10(2):217-243, 1970. 1018 8

[64] J.-L. Lions. Quelques méthodes de résolution des problèmes aux limites non linéaires. Dunod, 1969. 20.2

[65] J. Málek, J. Nečas, M. Rokyta and M. Ružička. Weak and measure-valued solutions to evolutionary PDEs. Chapman \& Hall, London, 1996. 11 2

[66] C. Mascia, A. Porretta and A. Terracina. Nonhomogeneous dirichlet problems for degenerate hyperbolic-parabolic equations. Arch. Ration. Mech. Anal. 163(2):87-124, 2002. 10

[67] A. Michel and J. Vovelle. Entropy formulation for parabolic degenerate equations with general Dirichlet boundary conditions and application to the convergence of FV methods. SIAM J. Numer. Anal. 41(6):2262-2293, 20031018

[68] M. Ohlberger. A posteriori error estimates for vertex centreed finite volume approximations of convection-diffusion-reaction equations. M2AN Math. Model. Numer. Anal. 35(2):355-387, 2001. 1

[69] F. Otto. Initial-boundary value problem for a scalar conservation law. C. R. Acad. Sci. Paris Sér. I Math. 322(8):729-734, 1996. 10

[70] F. Otto. $L^{1}$-contraction and uniqueness for quasilinear elliptic-parabolic equations. J. Differential Equations 131(1):20-38, 1996. 12

[71] E.Yu. Panov. On the theory of generalized entropy solutions of the Cauchy problem for a first-order quasilinear equation in the class of locally integrable functions. (Russian) Izvestiya Math. 66(6):1171-1218, 2002. 8

[72] Ch. Pierre.Modélisation et simulation de lactivité électrique du coeur dans le thorax, analyse numérique et méthodes de volumes finis. Ph.D. Thesis, Université de Nantes, 2005. 13.2

[73] É. Rouvre and G. Gagneux. Solution forte entropique de lois scalaires hyperboliquesparaboliques dégénérées. C. R. Acad. Sci. Paris Sér. I Math. 329(7):599-602, 1999. 101 8

[74] F. Simondon. Étude de l'équation $\partial_{t} b(u)-\operatorname{div} a(b(u), \nabla u)=0[\ldots]$ par la méthode des semi-groupes dans $L^{1}(\Omega)$. (French) Publ. Math. Fac. Sci. Besançon 7, Univ. Franche-Comté, Besançon, 1983. 1

[75] P. E. Souganidis and B. Perthame. Dissipative and entropy solutions to non-isotropic degenerate parabolic balance laws. Arch. Ration. Mech. Anal., 170(3):359-370, 2003; Addendum in Arch. Ration. Mech. Anal. 174,no.3:443-447, 2004. 1

[76] T. Tassa. Uniqueness of piecewise smooth weak solutions of multidimensional degenerate parabolic equations. J. Math. Anal. Applic. 210(2):598-608, 1997. 1

[77] A. I. Vol'pert. The spaces BV and quasi-linear equations. Math. USSR Sbornik, 2(2):225-267, 1967. 1

[78] A. I. Vol'pert and S. I. Hudjaev. Cauchy's problem for degenerate second order quasilinear parabolic equations. Math. USSR Sbornik 7(3):365-387, 1969. 1

[79] J. Vovelle. Convergence of finite volume monotone schemes for scalar conservation laws on bounded domains. Numer. Math. 90(3):563-596, 2002. 11 1

[80] Z. Wu and J. Yin. Some properties of functions in $B V_{x}$ and their applications to the uniqueness of solutions for degenerate quasilinear parabolic equations. Northeastern Math. J. 5(4):395-422, 1989. 1

[81] Z. Wu, J. Zhao, J. Yin and H. Li. Nonlinear diffusion equations. World Scientific Publishing Co. Inc., River Edge, NJ, 2001. 1 
[82] J. Yin. On a class of quasilinear parabolic equations of second order with double-degeneracy.

J. Partial Differential Equations 3(4):49-64, 1990. 1

(Boris Andreianov)

Laboratoire de Mathématiques

Université de Franche-Comté

16 ROUTE DE GRAY

25030 Besançon Cedex, France

E-mail address: boris.andreianov@univ-fcomte.fr

(Mostafa Bendahmane)

Departamento de Ingeniería Matemática

FaCUltad de Ciencias Físicas y Matemáticas

UNIVERSIDAD DE CONCEPCIÓN

Casilla 160-C Concepción, Chile

E-mail address: mostafab@ing-mat.udec.cl

(Kenneth H. Karlsen)

Centre of Mathematics for Applications

UNIVERSITY OF OSLO

P.O. Box 1053, BLINDERN

N-0316 Oslo, Norway

AND

Center for Biomedical Computing,

Simula Research laboratory

P.O. Box 134

N-1325 Lysaker, Norway

E-mail address: kennethk@math.uio.no

$U R L$ : http://folk.uio.no/kennethk 